MARIA TERESA DE MELLO R. SOUTO

Estudos clínicos da infusão contínua de fentanil, quetamina ou lidocaína sobre o requerimento de isoflurano em cavalos submetidos à cirurgia de artroscopia

São Paulo

2010 
MARIA TERESA DE MELLO R. SOUTO

\title{
Estudos clínicos da infusão contínua de fentanil, quetamina ou lidocaína sobre o requerimento de isoflurano em cavalos submetidos à cirurgia de artroscopia
}

\author{
Dissertação apresentado ao Programa de Pós- \\ Graduação em Clínica Cirurgia Veterinária da \\ Faculdade de Medicina Veterinária e Zootecnia da \\ Universidade de São Paulo, para obtenção do \\ título de mestre em Ciência \\ Departamento: \\ Cirurgia \\ Área de concentração: \\ Clínica Cirúrgica Veterinária \\ Orientador: \\ Profa Dra. Aline Magalhães Ambrósio
}

São Paulo 
Autorizo a reprodução parcial ou total desta obra, para fins acadêmicos, desde que citada a fonte.

DADOS INTERNACIONAIS DE CATALOGAÇÃO-NA-PUBLICAÇÃO

(Biblioteca Virginie Buff D’Ápice da Faculdade de Medicina Veterinária e Zootecnia da Universidade de São Paulo)

T.2298

FMVZ
Souto, Maria Teresa de Mello R.

Estudos clínicos da infusão contínua de fentanil, quetamina ou lidocaína sobre o requerimento de isoflurano em cavalos submetidos à cirurgia de artroscopia / Maria Teresa de Mello R. Souto. -- 2010.

125 f. : il.

Dissertação (Mestrado) - Universidade de São Paulo. Faculdade de Medicina Veterinária e Zootecnia. Departamento de Cirurgia, São Paulo, 2010.

Programa de Pós-Graduação: Clínica Cirurgia Veterinária.

Área de concentração: Clínica Cirurgia Veterinária.

Orientador: Profa. Dra. Aline Magalhães Ambrósio.

1. Fentanil. 2. Lidocaína. 3. Quetamina. 4. Infusão contínua. 5. Equinos. I. Título. 
UNIVERSIDADE DE SÃo PAULO

\section{Comissão Bioetica}

\section{CERTIFICADO}

Certificamos que o Projeto intitulado "Efeitos da infusão contínua de fentanil sobre a concentração alveolar mínima do isoflurano, sistema cardiovascular, respiratório e na recuperação em eqüinos submetidos a artroscopia", protocolado sob o n¹489/2008, utilizando 15 cavalos, sob a responsabilidade da Profa. Dra. Aline Magalhães Ambrósio, está de acordo com os princípios éticos de experimentação animal da Comissão de Bioética da Faculdade de Medicina Veterinária e Zootecnia da Universidade de São Paulo e foi aprovado em reunião de $01 / 10 / 08$.

We certify that the Research "Effects of continuous infusion fentanyl on the minimum alveolar concentration, cardiovascular and respiratory system and recovery of horses submitted to arthroscopy", protocol number 1489/2008, utilizing 15 (fifteen) horses, under the responsibility Profa. Dra. Aline Magalhães Ambrósio, agree with Ethical Principles in Animal Research adopted by Bioethic Commission of the School of Veterinary Medicine and Animal Science of University of São Paulo and was approved in the meeting of day 10/01/08.

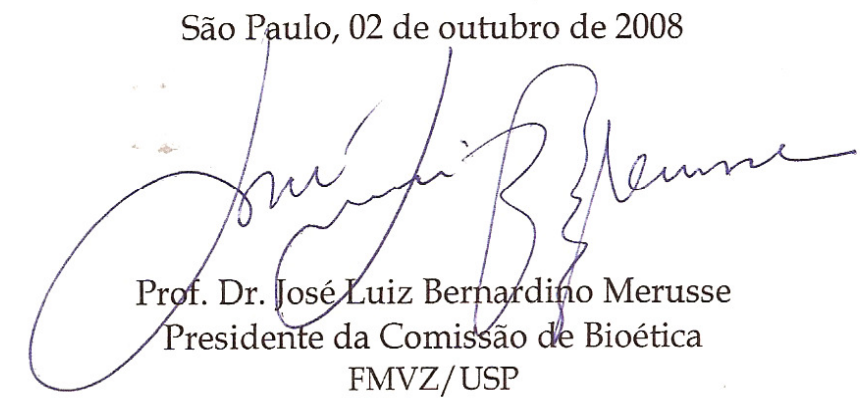


Nome: SOUTO, Maria Teresa de Mello R.

Título: Estudos clínicos da infusão contínua de fentanil, quetamina ou lidocaína sobre o requerimento de isoflurano em cavalos submetidos à cirurgia de artroscopia

Dissertação apresentada ao Programa de Pós-Graduação em Clínica Cirurgica Veterinária da Faculdade de Medicina Veterinária e Zootecnia da Universidade de São Paulo para obtenção do título de Mestre em Ciências

Data:

Banca Examinadora

Prof. Dr.

Instituição:

Julgamento:

Assinatura:

Prof. Dr.

Instituição:

Julgamento:

Assinatura:

Prof. Dr.

Instituição:

Julgamento:

Assinatura: 


\section{DEDICATÓRIA}

Dedico este trabalho,

A meu marido Edson Garcia Tosta, sem ele nenhum sonho seria possível ou valeria à pena.

A minhas filhas Ana Beatriz e Giuliana, as quais dedico minha vida.

Ao meu pai Massilon e minha mãe Teresa Cristina, que um dia sonharam e hoje compartilham este importante momento comigo. 
O saber a gente aprende com os mestres e os livros. A sabedoria se aprende é com a vida e com os humildes."

Cora Coralina 


\section{AGRADECIMENTOS}

A minha irmã Christiane, por ter sido uma árdua incentivadora na realização deste mestrado.

A minha avó Ezinha, que em seus 94 anos ainda é minha fonte de expiração e um exemplo a ser seguido, por sua força e amor incondicional.

À professora Denise Fantoni, por ter acreditado em mim, ter me recebido nesta instituição e ter contribuído, com seus ensinamentos, para o enriquecimento dos meus conhecimentos, pelo carinho e amizade.

À professora Aline Magalhães Ambrósio, por me receber como orientada, pela ajuda, apoio e confiança em todos os momentos. Pelos conhecimentos transmitidos a mim durante estes quatro anos de convivência e pela amizade construída.

À professora Silvia Gaido Coropassi, pelo carinho, interesse constante em ajudar, o apoio e a amizade.

Ao professor Luis Claudio Lopes Correia da Silva, pela contribuição na realização deste experimento, pelo carinho e compreensão em todas as cirurgias de artroscopia que foram de fundamental importância para a conclusão deste estudo. 
Ao professor André Luiz do Valle De Zoppa, pela amizade e pelo carinho, além é claro dos momentos de descontração.

Aos enfermeiros Henrique Fragoso, Cícero Antônio da Silva e Marcos Roberto Rodrigues Alves por estarem sempre dispostos a contribuir e pelo fundamental auxílio para a execução deste projeto, além da amizade e carinho.

Aos residentes do Hospital Veterinário de grandes animais, que de alguma forma contribuíram na execução deste experimento.

À pós-graduanda Thaís Sodré de Machado, pela amizade, carinho e disposição para ajudar sempre que precisei.

Aos pós-graduandos Keila Kazue Ida , Julio David Spagnolo e Gustavo Miranda Zanotto, pelo apóio e carinho em todo o decorrer deste experimento.

À pós-graduanda Larissa Borges Cardoso, pela ajuda fundamental em todo meu experimento, pelo carinho, amizade e muito incentivo.

À médica veterinária Lara Lopes Faco Mott, pela ajuda, amizade e dedicação ao meu experimento.

À Médica Veterinária Dra. Márcia Kahvegian, quem admiro muito, pelo apoio constante, se não fosse sua vontade, empenho, disposição para ajudar e intermináveis incentivos, esta dissertação não seria completa. 
À técnica em laboratório do banco de sangue Selene Aparecida de Oliveira,pelas vezes que precisei da ajuda nas análises de hemogasometria.

Ao técnico em laboratório, José Miron Oliveira da Silva, também pela ajuda nas análises de hemogasometria.

A amiga Cristiane, professora das minhas filhas, pela ajuda constante com a Aninha e a Juju em todos os momentos que precisei ficar ausente, pela paciência e dedicação a elas, fazendo com que fosse possível a realização deste estudo.

Aos funcionários Alessandra Ap. A. de Sousa e Berlamino Ney Pereira, pela paciência e a disposição de ajudar a resolver nossos problemas.

A todos os cavalos que foram submetidos à cirurgia de artroscopia, sendo o alvo da minha pesquisa, os quais ajudaram a realização deste experimento.

A todos os amigos que me incentivaram, dando apóio na decisão da realização deste mestrado. 


\section{RESUMO}

SOUTO, M. T. Avaliação clínica dos efeitos da infusão contínua de fentanil, quetamina ou lidocaína sobre o requerimento de isoflurano em cavalos submetidos à cirurgia de artroscopia. [Clinical evaluation of the effects of continuous infusion of fentanyl, ketamine or lidocaine on the requirement of isoflurane in horses undergoing arthroscopic surgery]. 2010. 125 f. Dissertação (Mestrado em Ciências) - Faculdade de Medicina Veterinária e Zootecnia, Universidade de São Paulo, São Paulo, 2010.

Os equinos são comumente anestesiados com agentes voláteis em procedimentos cirúrgicos acima de 60 minutos, resultando em depressão cardiovascular dosedependente, contribuindo para uma alta taxa de mortalidade. Sendo assim, este estudo objetivou determinar se o fentanil, em infusão contínua, seria capaz de diminuir a fração expirada de isoflurano, promovendo estabilidade cardiovascular transoperatória e uma melhor recuperação após cirurgia de artroscopia, em comparação à infusão de lidocaína ou quetamina, durante a manutenção da anestesia com isoflurano. Para tanto foram utilizados 20 equinos de 3 a 8 anos e pesando 350 a $500 \mathrm{~kg}$, submetidos a cirurgia de artroscopia em decúbito dorsal. Os animais foram divididos aleatoriamente em 4 grupos: $\mathrm{GL}(1,5 \mathrm{mg} / \mathrm{kg}$ em bolus e infusão de

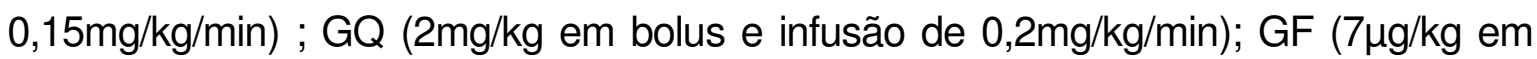
bolus e infusão de $0,7 \mu \mathrm{g} / \mathrm{kg} / \mathrm{min}$ ) e Gl que não recebeu infusão de nenhum fármaco adjuvante. Foram avaliados os parâmetros cardiovasculares (FC, PAM, PAS e PAD), ventilatórios e de oxigenação $\left(\mathrm{PaO}_{2}, \mathrm{PaCO}_{2}\right.$, Compl e Rva), fração expirada de isoflurano [ISSO](Fexp\%), e qualidade de recuperação. Em relação à [ISSO] (Fexp\%) as maiores quedas foram observadas no momento 30bolus em todos os grupos, que utilizaram adjuvantes, comparados ao momento basal sendo, GL (1,50 para 0,90\%) GQ (1,44 para 0,96\%) e GF (1,32 para 0,96\%). Observou-se que após 15 minutos da interrupção da infusão contínua de fentanil e lidocaína a fração expirada de isoflurano foi aumentada GL 25\% e GF 45\%. Apesar de não ter havido diferença estatística, o GF apresentou escore de recuperação menor 16,8 pontos, GL 24,6; GQ 30,0 e GI 31,8 pontos. Conclui-se então que o uso do fentanil foi capaz de reduzir a fração expirada de isoflurano em até $43 \%$, não observando qualquer efeito colateral no momento da recuperação após re-sedação com xilazina $0,5 \mathrm{mg} / \mathrm{kg}$.

Palavras-chave: Fentanil. Lidocaína. Quetamina. Infusão contínua. Equinos. 


\begin{abstract}
SOUTO, M. T. Clinical evaluation of the effects of continuous infusion of fentanyl, ketamine or lidocaine on the requirement of isoflurane in horses undergoing arthroscopic surgery. [Avaliação clínica dos efeitos da infusão contínua de fentanil, quetamina ou lidocaína sobre o requerimento de isoflurano em cavalos submetidos à cirurgia de artroscopia]. 2010. 125 f. Dissertação (Mestrado em Ciências) - Faculdade de Medicina Veterinária e Zootecnia, Universidade de São Paulo, São Paulo, 2010.
\end{abstract}

Volatile anesthetics are commonly used in horse anesthesia in surgical procedures over 60 minutes, resulting in a dose-dependent cardiovascular depression, contributing to a high mortality rate. Thus, the aimed this study was determine whether fentanyl continuous rate infusion would be able to reduce end tidal isoflurane, promoting intraoperative cardiovascular stability and a better recovery after arthroscopic surgery, when compared to lidocaine or ketamine infusion during maintenance of anesthesia with isoflurane. Therefore, 20 horses aging 3-8 years and weighing 350 to $500 \mathrm{~kg}$ underwent arthroscopic surgery in dorsal recumbence. The animals were randomly divided into four groups: GL (1.5 $\mathrm{mg} / \mathrm{kg}$ bolus and $0.15 \mathrm{mg} / \mathrm{kg} / \mathrm{min}$ infusion rate of lidocaine), GQ (2 mg/kg bolus and $0.2 \mathrm{mg} / \mathrm{kg} / \mathrm{min}$ infusion of ketamine); GF $(7 \mu \mathrm{g} / \mathrm{kg}$ bolus and $0.7 \mathrm{mg} / \mathrm{kg} / \mathrm{min}$ infusion rate of fentanyl) and $\mathrm{Gl}$ did not receive any adjuvant infusion. Cardiovascular parameters (HR, MBP, SBP and DBP), ventilatory and oxygenation (PaO2, $\mathrm{PaCO} 2, \mathrm{Cst}$ and Rva), end tidal isoflurano [ISO] (Fexp\%), and quality of recovery were evaluated. Regarding [ISO] (Fexp\%) the highest decreases were observed at the time 30bolus in all groups with adjuvants, in comparison to base line -GL (1.50 to $0.90 \%) \mathrm{GQ}(1.44$ to $0.96 \%)$ and GF (1.32 to $0.96 \%)$. At 15 minutes after the end of continuous rate infusion of fentanyl and lidocaine, expired fraction of isoflurane was increased $25 \%$ for $G L$ and $45 \%$ for GF. Although there was no statistical difference, GF showed lower recovery score - 16.8 points, while 24.6 for GL, 30.0 for $\mathrm{GQ}$ and 31.8 points for GI. In conclusion, fentanyl was able to reduce end tidal isoflurane to $43 \%$, with no side effects at recovery time after re-sedation with xylazine $0.5 \mathrm{mg} / \mathrm{kg}$.

Keywords: Fentanyl. Lidocaine. Ketamine. Continuous infusion. Horses. 


\section{LISTA DE QUADROS}

Quadro 1 - Escala de avaliação da qualidade de recuperação, Donaldson et al. (2000)

Quadro 2- Valores referentes a peso, consumo de efedrina, taxa de infusão de fluido e diurese dos grupos: lidocaína $(G L)$, quetamina (GQ), fentanil (GF) e isoflurano (Gl) (Média \pm Desvio Padrão) - São Paulo - 2009... 


\section{LISTA DE TABELAS}

Tabela 1 - Parâmetros hemodinâmicos diretos dos animais dos grupos lidocaína (GL), quetamina (GQ), fentanil (GF) e isoflurano (Gl) (Média \pm Desvio Padrão) - São Paulo - 2009.

Tabela 2 - Parâmetros de oxigenação e fração expirada de isoflurano dos animais dos grupos lidocaína (GL), quetamina (GQ), fentanil (GF) e isoflurano (Gl) (Média \pm Desvio Padrão) - São Paulo 2009

Tabela 3 - Parâmetros metabólicos dos animais dos grupos lidocaína (GL), quetamina (GQ), fentanil (GF) e isoflurano (Gl) (Média \pm Desvio Padrão) - São Paulo - 2009

Tabela 4 - Parâmetros de ventilação dos animais dos grupos lidocaína (GL), quetamina (GQ), fentanil (GF) e isoflurano (GI) (Média \pm Desvio Padrão) - São Paulo - 2009 


\section{LISTA DE GRÁFICOS}

Gráfico 1 - Média e Desvio Padrão da frequência cardíaca (batimentos por minuto) dos animais dos grupos: lidocaína (GL), quetamina $(G Q)$, fentanil (GF) e isoflurano (GI) - São Paulo - 2009.

Gráfico 2 - Média e Desvio Padrão da pressão arterial sistólica $(\mathrm{mmHg})$ dos animais dos grupos: lidocaína $(\mathrm{GL})$, quetamina $(\mathrm{GQ})$, fentanil (GF) e isoflurano (Gl) (Média \pm Desvio Padrão) - São Paulo 2009 .

Gráfico 3 - Média e Desvio Padrão da pressão arterial média $(\mathrm{mmHg})$ dos animais dos grupos: lidocaína (GL), quetamina (GQ), fentanil (GF) e isoflurano (GI) (Média \pm Desvio Padrão) - São Paulo 2009 .

Gráfico 4 - Média e Desvio Padrão da pressão arterial diastólica $(\mathrm{mmHg})$ dos animais dos grupos: lidocaína (GL), quetamina (GQ), fentanil (GF) e isoflurano (Gl) (Média \pm Desvio Padrão) - São Paulo - 2009

Gráfico 5 - Média e Desvio Padrão da fração expirada de isoflurano (Fexp\%) dos animais dos grupos: lidocaína (GL), quetamina (GQ), fentanil (GF) e isoflurano (Gl) (Média \pm Desvio Padrão) São Paulo - 2009

Gráfico 6 - Média e Desvio Padrão da taxa de infusão de fluido $(\mathrm{ml} / \mathrm{Kg} / \mathrm{h})$ dos animais dos grupos: lidocaína (GL), quetamina (GQ), fentanil (GF) e isoflurano (Gl) (Média \pm Desvio Padrão) - São Paulo - 2009

Gráfico 7 - Média e Desvio Padrão da taxa consumo de efedrina (mg) dos animais dos grupos: lidocaína $(G L)$, quetamina $(G Q)$, fentanil (GF) e isoflurano (Gl) (Média \pm Desvio Padrão) - São Paulo 2009

Gráfico 8 - Média e Desvio Padrão da diurese $(\mathrm{ml} / \mathrm{Kg} / \mathrm{h})$ dos animais dos grupos: lidocaína (GL), quetamina (GQ), fentanil (GF) e isoflurano (Gl) (Média \pm Desvio Padrão) - São Paulo 2009. 
Gráfico 9 - Média e Desvio Padrão da complacência estática $\left(\mathrm{ml} / \mathrm{cmH}_{2} \mathrm{O}\right)$ dos animais dos grupos: lidocaína (GL), quetamina (GQ), fentanil (GF) e isoflurano (Gl) (Média \pm Desvio Padrão) - São Paulo - 2009.

Gráfico 10 Média e Desvio Padrão do escore de recuperação dos animais dos grupos: lidocaína (GL), quetamina (GQ), fentanil (GF) e isoflurano (Gl) (Média \pm Desvio Padrão) - São Paulo 2009. 


\section{LISTA DE ABREVIATURAS E SIGLAS}

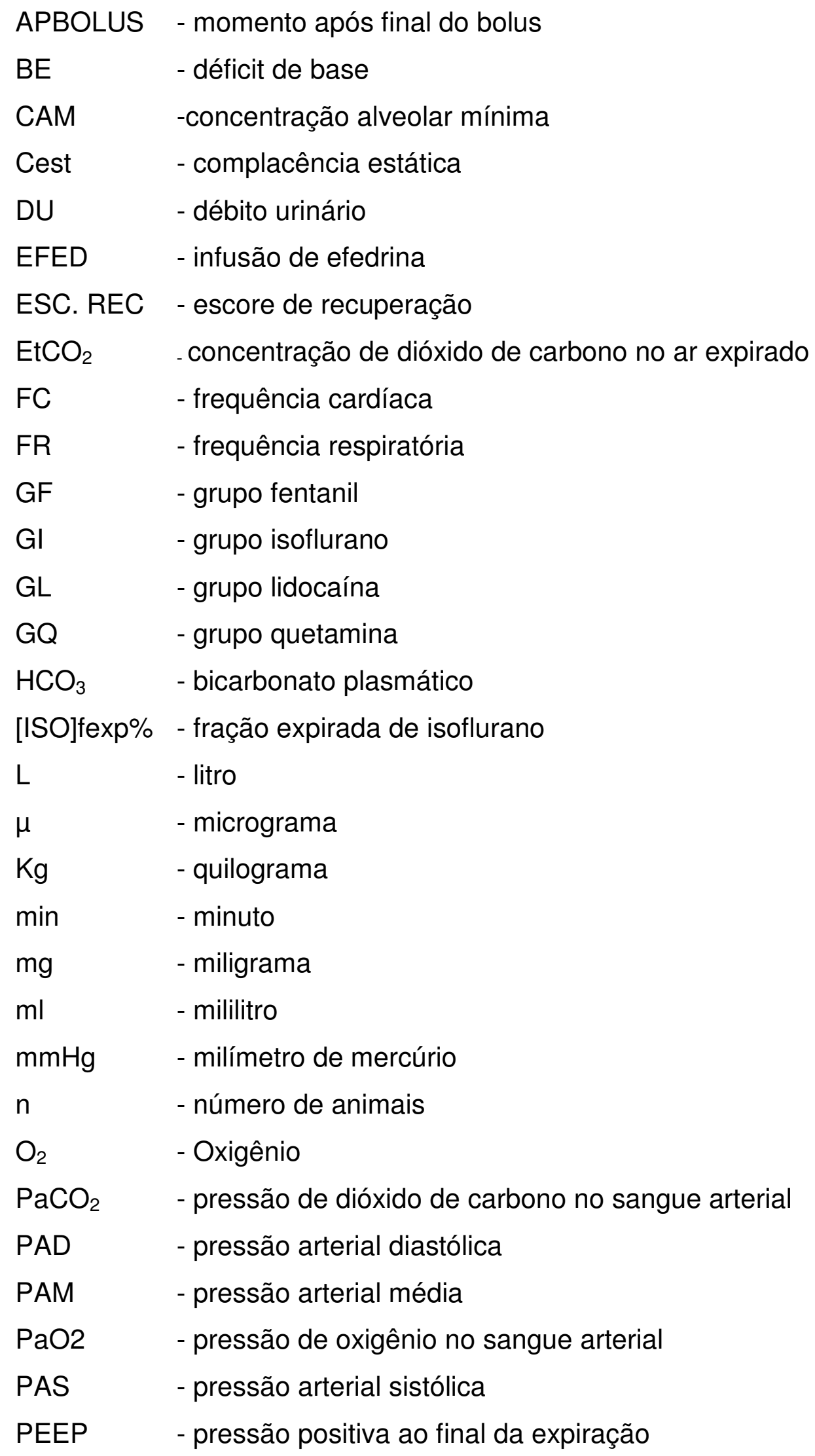



Ppico - -pressão de pico inspiratório
Pplatô - pressão de platô
Rva - resistência das vias aéreas
$\mathrm{SaO}_{2}$ - saturação arterial de hoxihemoglobina
SNC - sistema nervoso central
$\mathrm{T}^{\circ} \mathrm{C} \quad$ - temperatura
Vex - volume expirado
20 INAL - momento 20 minutos após o início da anestesia inalatória
15 BOLUS - momento após 15 minutos do final do bolus
30 BOLUS -momento após 30 minutos do final do bolus
45 BOLUS - momento após 45 minutos do final do bolus
75 BOLUS - momento após 75 minutos do final do bolus 


\section{SUMÁRIO}

1 INTRODUÇÃO

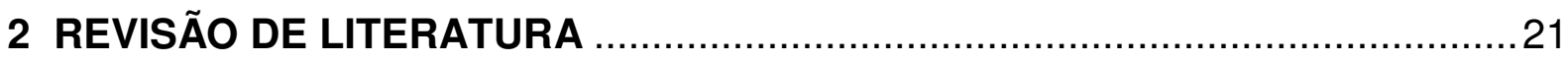

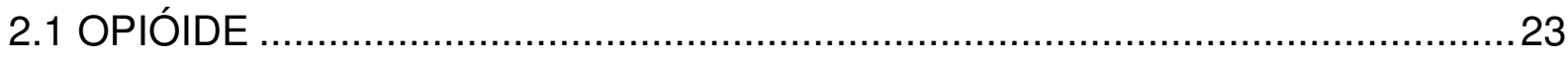

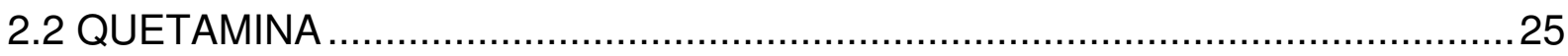

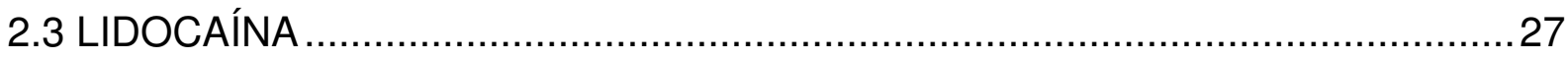

3 JUSTIFICATIVA

4 OBJETIVOS

5 MATERIAL E MÉTODO

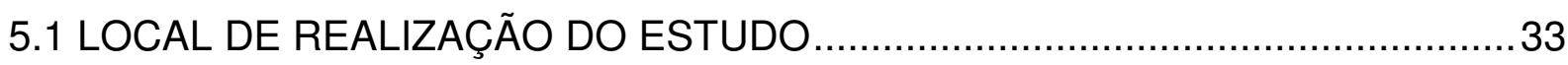

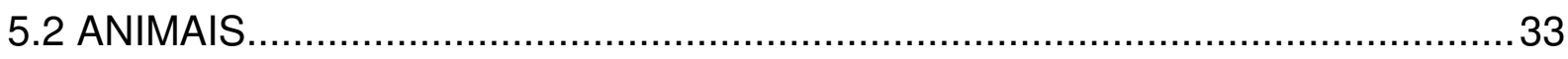

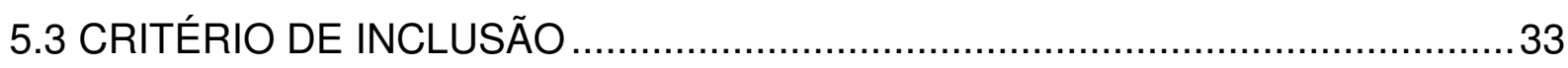

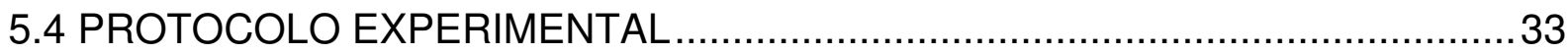

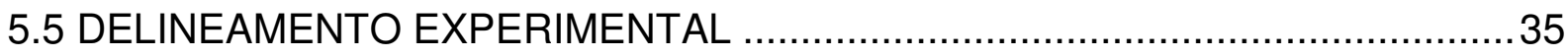

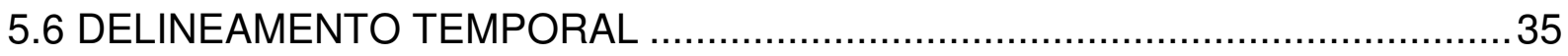

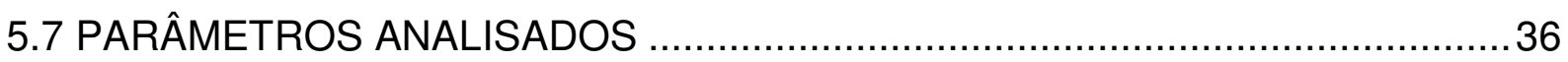

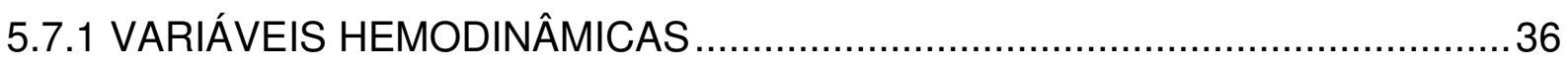

5.7.2 VARIÁVEIS DE OXIGENAÇÃO, VENTILAÇÃO E MECÂNICA PULMONAR ..36

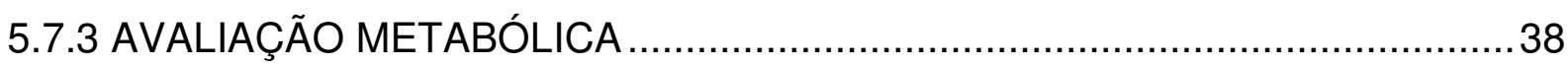

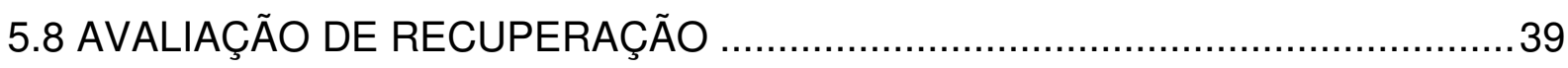

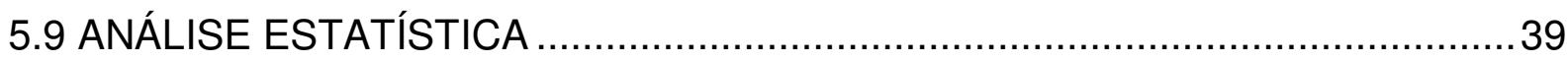

6 RESULTADOS

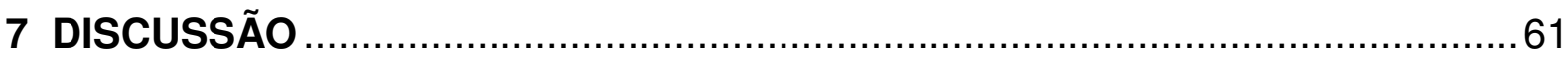

8 CONCLUSÃO

REFERÊNCIAS

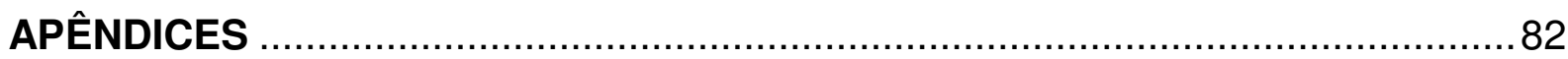




\section{INTRODUÇÃO}

Cavalos submetidos à anestesia para procedimentos prolongados são usualmente mantidos com agentes anestésicos voláteis, utilizados por apresentarem fácil controle de administração, não possuírem limite definido de duração de uso e pelas suas características de rápida indução e recuperação, porém a depressão cardiovascular provocada por eles é dose dependente (STEFFEY; HOWLAND, 1978, 1980).

O Isoflurano é hoje o agente anestésico inalatório mais amplamente utilizado em anestesia eqüina, por ser um agente não inflamável, facilmente vaporizado e de baixa solubilidade no sangue e na gordura. Este agente anestésico pode ser utilizado na anestesia de pacientes debilitados, pois possui baixo risco de sobredose e baixa taxa de metabolização $(0,2 \%)$, podendo, portanto, ser empregado em pacientes hepatopatas e nefropatas. A concentração alveolar média (CAM) deste fármaco em eqüinos é de 1,4\% porém, ela pode ser afetada por diversos fatores, que podem diminuí-la ou aumentá-la. Para prevenir movimentos durante o procedimento cirúrgico geralmente é necessário manter a concentração expirada final do anestésico inalatório cerca de $30 \%$ maior do que a CAM (STEFFEY; HOWLAND, 1978). Sabe-se também que fármacos analgésicos e sedativos geralmente permitem diminuir a concentração utilizada. Outro fator que pode alterar a CAM é a utilização de medicamento pré-anestésicos (MPA), por serem fármacos depressores do sistema nervoso central (SNC) (STEFFEY et al., 2000; YAMASHITA, 2009).

Alguns anestésicos injetáveis por possuírem ação analgésica, como quetamina e o anestésico local lidocaína podem ser associadas a anestésicos inalatórios como alternativa para manter um melhor desempenho cardiopulmonar em anestesia inalatória para suprimir as respostas endócrinas ao estresse observadas e diminuir a CAM dos anestésicos inalatórios durante anestesia com halotano (LUNA; TAYLOR; MASSONE, 1996).

As anestesias balanceadas com a utilização de opióides, anestésicos dissociativos em baixa dose e lidocaína, podem ser alternativas para diminuir a CAM do anestésico inalatório, fato já constatado na anestesia de pequenos animais e no 
homem. Em eqüinos os efeitos dos opióides ainda são pouco conhecidos e controversos, entretanto, a metadona e a petidina tem sido usados em cavalos por várias décadas na Europa e não existe dúvida que os opióides são efetivos também nesta espécie (TAYLOR; PASCOE; MAMA, 2007). 


\section{REVISÃO DE LITERATURA}

A literatura indica alta morbidade e mortalidade perioperatória associada com anestesia em cavalos (1\%) (JOHNSTON et al., 2002) quando comparado com seres humanos (0,01\%) (LUNN et al, 1982) e cães e gatos (0,07\%) (DYSON et al., 1998). Neste sentido, têm sido realizados muitos estudo acerca dos efeitos dos anestésicos na função cardiovascular de equinos e sua contribuição para a mortalidade perioperatória (JOHNSTON et al., 2002).

Os cavalos são comumente anestesiados com anestésicos voláteis como halotano e isoflurano em procedimentos cirúrgicos acima de 60 minutos, o que comumente resulta em depressão cardiovascular dose-dependente, vasodilatação, hipotensão e baixos valores do débito cardíaco, contribuindo para uma alta taxa de mortalidade (STEFFEY; HOWLANDI., 1980).

Os anestésicos inalatórios, além de promoverem os fatores essenciais para realização de anestesia cirúrgica, como hipnose, bloqueio dos reflexos autonômicos e relaxamento muscular, produzem efeitos indesejáveis, como depressão do sistema circulatório e respiratório, os quais são associados às complicações nos períodos trans e pós-operatório (JOHNSON et al., 1995; FANTONI; CORTOPASSI; BERNARDI, 1999).

Estudos em diversas espécies incluindo cavalos indicam que 0 isoflurano apresenta uma grande margem de segurança cardiovascular quando comparado ao halotano (STEFFEY, 1982). Blissitt et al. (2008) demonstrou um índice cardíaco superior com o uso do isoflurano quando comparado ao halotano em cavalos submetidos à cirurgias eletivas em diferentes modos de ventilação. Possivelmente a maior depressão cardiovascular promovida pela halotano é devido à diminuição da contratilidade associada a este fármaco (BRUNSON et al., 1990).

As características anestésicas desejáveis para os procedimentos cirúrgicos, como a tríade anestésica, relacionada à inconsciência, relaxamento muscular e analgesia, requerem um plano anestésico profundo, o qual normalmente vem acompanhado pela severa depressão da perfusão tecidual. Sendo assim, a monitoração da função cardiovascular durante a anestesia geral eqüina é primordial para garantia de um procedimento seguro, pois permite um rápido reconhecimento 
de intercorrências e a interferência imediata, antes do desenvolvimento de lesão por hipóxia (KEEGAN; GREENE, 1994; HESS, 2000).

$O$ aumento na freqüência cardíaca parece ser uma característica do isoflurano em pequenos animais (DUKET; CAULKETT; TATARYN, 2006), fato também observado em estudo com potros (DUNLOP et al., 1990 ). Entretanto, Aida et al. (2000) demonstrou um aumento significativamente maior da freqüência cardíaca com o sevoflurano, quando comparado ao halotano e ao isofluorano em equinos submetidos à ventilação controlada e ao uso de $\alpha-2$ agonista.

A pressão arterial diminui com o uso do isoflurano e a magnitude desta diminuição é dose dependente em cavalos adultos (STEFFEY; HOWLAND, 1980) e em potros (MANOHAR; GOETZ, 1985; DUNLOP et al., 1990).

A manutenção da pressão arterial em valores adequados tem sua importância reconhecida na garantia de uma boa perfusão tecidual (WAGNER; BRODBELT, 1997; TRIM, 1998). A terapia para garantir a perfusão tecidual abrange diminuição da concentração inspirada de halogenado, fluidoterapia intravenosa e melhora do débito cardíaco por aumento da freqüência cardíaca ou da contratilidade cardíaca por meio do uso de agentes inotrópicos e/ou vasopressores (MUIR, 2009).

Agentes inalatórios por afetarem significativamente a perfusão tecidual, podem comprometer o aporte de oxigênio, assim como, a remoção do dióxido de carbono tecidual. Nesse sentido, qualquer comprometimento no sistema circulatório pode resultar em dano cerebral, falência renal ou isquemia muscular (WAGNER; BRODBELT, 1997).

A hipotensão e, consequentemente, a perfusão tecidual inadequada promovem maior incidência de miopatias pós-anestésica em equinos, além de íleo paralítico e insuficiência renal (KEEGAN; GREENE,1994; TRIM, 1998). A miopatia pós-anestésica é apontada como a responsável por 8 a $60 \%$ da mortalidade póscirúrgica imediata nessa espécie (TEVIK, 1983). Desta maneira, como a perfusão tecidual é reduzida pela maioria dos agentes anestésicos gerais empregados, sendo tal redução atribuída à vasodilatação, diminuição do débito cardíaco e hipovolemia, os esforços devem ser direcionados na tentativa de se reduzir a concentração de anestésicos halogenados, durante os procedimentos cirúrgicos nessa espécie ( MARCHIONI, 2003).

Analgésicos intravenosos ou anestésicos adjuvantes podem ser administrados durante a anestesia inalatória para minimizar o requerimento do 
agente anestésico e assegurar o alívio da dor nos períodos trans e pós operatório (YAMASHITA et al., 2009, além de suprimir a resposta de estresse endócrino durante a anestesia (LUNA et al., 1996). A combinação de anestesia inalatória e agentes injetáveis promovem benefícios como adequado plano de anestesia cirúrgica, redução da depressão cardiopulmonar e rápida recuperação anestésica (SPADAVECCHIA et al., 2002; KUSHIRO et al., 2005).

O conceito de combinar vários componentes com diferentes ações, como amnésia, analgesia e diminuição dos reflexos autonômicos, foi primeiro relatado por George W. Crile, em 1910 em seres humanos, que sugeriu o uso de uma leve anestesia geral junto com anestesia local para bloquear o estímulo doloroso. 0 termo anestesia balanceada foi introduzido por John S. Lundy em 1926, cuja idéia foi utilizar diferentes agentes e técnicas para alcançar diferentes objetivos durante a anestesia. O atual conceito de anestesia balanceada consiste na combinação de diferentes fármacos e diferentes efeitos com mínimos efeitos adversos (BETTSCHART-WOLFENSBERGER et al., 2007).

A anestesia balanceada em equinos tem como objetivo manter a função cardiopulmonar estável seguida por uma recuperação tranquila e coordenada, consistindo no uso concomitante de múltiplos fármacos anestésicos com o potencial de reduzir o requerimento de anestésicos inalatórios (MUIR, 2009). Sendo assim, a administração intravenosa de opioides (CLARK et al., 2005; TOMASY et al., 2007) quetamina ( MUIR et al.,1992) e lidocaina (DZITIKI et al., 2003; VALVERDE et al., 2005) tem sido investigada na referida espécie, durante a anestesia inalatória.

\subsection{OPIÓIDE}

Os opióides atualmente são muito empregados em Medicina Veterinária, sendo indicados para o tratamento da dor em diferentes situações, pois são considerados agentes com alta eficácia e segurança. Após o intervalo de 20 anos, estes fármacos voltaram a ser utilizados corriqueiramente na década de 1990 e atualmente empregam-se cerca de dez diferentes agentes opióides para o tratamento da dor de várias etiologias e em diversos momentos de período perioperatório (FANTONI et al., 2010).

Esses fármacos ligam-se reversivelmente a receptores específicos no SNC e medula espinhal e produzem analgesia através da interação com receptores pós - 
sinápticos específicos no sistema nervoso central e em outros tecidos que também sejam locais de ação de determinados peptídeos endógenos, como encefalinas, endorfinas e dimorfinas (GOODMAN et al., 1987).

O efeito adverso mais importante dos opióides é a depressão respiratória, a qual é dose dependente. Neste sentido aconselha-se utilizar os opióides potentes no período transoperatório quando o animal está intubado e a ventilação mecânica instaurada (PAPICH, 1997).

Devido à presença de receptores opióides no trato gastrointestinal pode haver constipação, mediada por diminuição da contração da musculatura lisa intestinal, o que diminui o tempo de trânsito gastrointestinal, e a absorção de água e eletrólitos também podem se apresentar diminuídas (PAPICH, 1997).

Os opióides de um modo geral causam poucos efeitos adversos no sistema cardiovascular. O efeito mais descrito é a bradicardia, mediada por estímulo vagal. A hipotensão pode existir mediante a presença de bradicardia, liberação de histamina, observada principalmente com meperidina e morfina, depressão miocárdica e efeito simpatolítico (THURMON; TRANQUILLI; BENSON, 1996; GORNIAK, 2002).

O fentanil é um opióide agonista sintético de $\mu$-receptor e o analgésico mais comumente usado em seres humanos e em pequenos animais (THOMASY et al., 2007). Como analgésico, estima-se que seja 100 vezes mais potente que a morfina pela via intravenosa (PALMER et al., 1998). Ainda em análise comparativa com a morfina, o fentanil é mais potente e mais lipossolúvel e exibe um rápido início de ação e um período hábil de curta duração (MUIJSERS; WAGSTAFF, 2001). A analgesia, sedação e depressão respiratória ocorrem após quatro minutos da administração intravenosa, com pico de ação entre 10 a 15 minutos e duração de ação de 30 minutos (SOMA; SHIELDS, 1964).

Outros efeitos do fentanil são apnéia, salivação, bradicardia e relaxamento do esfíncter anal, com ocasional defecação (SOMA; SHIELDS, 1964; LAMONT et al., 2007). A bradicardia é desencadeada por estímulação vagal, não alterando a pressão arterial, nem o débito cardíaco o que explica a manutensão da perfusão capilar dos animais menor ou igual a 2 segundos (LAMONT et al., 2007) e a depressão respiratória é devido ao seu efeito sobre o centro respiratório (GRANDY et al., 1985). Da mesma forma o volume minuto diminui, sendo esta redução apenas significativa após a indução anestésica (MUIR et al., 1989; LAMO et al., 2007), o que parece estar relacionada com a redução da complacência torácica após a 
administração de grandes doses de fentanil intravenoso (GRANDY; STEFFEY, 1985).

Dentre os fármacos opióides o fentanil é o mais usado devido a suas propriedades físico-quimicas, tais como: baixo peso molecular, grande lipossolubilidade, e afinidade específica pelos receptores $\mu$ (COHEN; MAO,1992), sendo o fármaco de escolha em pediatria em função de sua margem de segurança e dos efeitos benéficos na estabilidade hemodinâmica (HICKEY et al., 1985 ; YASTER; KOEHLER; TRAYTMAN, 1987).

A administração intravenosa do fentanil tem sido descrita na diminuição da concentração alveolar mínima dos anestésicos inalatórios em humanos, cães e porcos, mas seu efeito em cavalos não tem sido muito documentado (MC EWAN et al., 1993; MOON et al., 1995). Entretanto, alguns estudos têm demonstrado que a morfina e o alfentanil não diminuem consistentemente a CAM dos anestésicos inalatórios em cavalos (PASCOE et al., 1993; STEFFEY; EISELE; BOGGOT, 2003).

Estudos clínicos preliminares avaliaram a eficácia do fentanil transdérmico na concentração plasmática de 1 a $2 \mathrm{ng} / \mathrm{ml}$ em cavalos adultos e potros, acordados, sugerindo que este promove uma analgesia significante sem apresentar efeitos colaterais. (THOMASY et al., 2004; EBERSPÄCHER et al. 2008). Segundo Thomasy et al. (2006), a concentração plasmática do fentanil intravenoso de $13 \mathrm{ng} / \mathrm{ml}$ em cavalos anestesiados tem demonstrado uma diminuição da concentração alveolar mínima de isoflurano de $18 \%$ e sugere que sua potência anestésica nesta espécie é similar a dos suínos e menor que no homem e em cães.

\subsection{QUETAMINA}

A quetamina surgiu em 1963 em substituição a fenciclidina, com o objetivo de produzir menor intensidade de reação adversas(FANTONI, 2010). Este fármaco possui potente poder analgésico e hipnótico, porém apresenta efeitos colaterais que resultam de sua ação dissociativa sobre o sistema nervoso central (SNC) e de sua interferência no sistema nervoso autônomo. Entre estes efeitos estão, alucinações, sialorréia, rigidez muscular, taquicardia e hipertensão arterial (CLEMENTS; NIMMO, 1981; SCHATZMANN; GIRARD, 1984).

A quetamina age como antagonista não-competitivo do complexo canalreceptor N-Metil-D-Aspartato. O resultado é o efeito analgésico potente, mesmo em 
doses subanestésicas (DIKENSON, 1996). Além disso, a quetamina também possui ação comprovada em diversos outros receptores tais como muscarínicos, nicotínicos, opióides mu, delta e kapa, monoaminérgicos e canais de cálcio voltagem-dependentes. Exerce ainda bloqueios nos canais de sódio do SNC e do sistema nervoso periférico (ARENDT et al., 1995).

A quetamina provoca mudanças consistentes no eletroencefalograma, diminuindo a atividade $\alpha$ e aumentando a atividade $\theta$ e $\Delta$ além de induzir atividades excitatórias que ocorre no tálamo e sistema límbico. Aumenta a pressão do líquor cefalorraquidiano e pressão intracraniana, que pode ser decorrente do aumento da concentração arterial de dióxido de carbono, provocada pela depressão respiratória, aumentando o fluxo sanguíneo cerebral e o consumo de oxigênio. Porém, segundo Valadão (2010), devido a quetamina ser um antagonista do receptor do tipo NMDA, em baixas doses, reduz seletivamente a excitação produzida pelos aminoácidos excitatórios, em neurônios do sistema nervoso central, possuindo então, ação anticonvulsivante e neuroprotetora. Sendo assim, a contradição encontrada na literatura referente a seus efeitos convulsivantes ou anticonvulsivantes, está relacionada a diferentes doses deste fármaco aliado ao emprego em diferentes espécies animais.

Este fármaco ainda produz aumento na frequência cardíaca, do tônus simpático, do débito cardíaco, da pressão arterial média, da pressão arterial pulmonar e da pressão venosa central. (MUIR et al., 1992; VALADÃO, 2010). Tende a estimular indiretamente o sistema cardiovascular, exercendo efeito inotrópico positivo seletivo na musculatura cardíaca, independentemente da frequência cardíaca e do sistema nervoso autonômico, podendo ser decorrente da alteração do monofosfato de adenosina cíclico intracelular. Os efeitos na resistência vascular periférica são variáveis; doses elevadas de quetamina, especialmente quando administrada por via intravenosa, podem causar efeitos depressores no sistema cardiovascular (LUMB; JOHNE'S, 2007).

Os efeitos estimulantes da quetamina no sistema cardiovascular podem ser diminuídos ou prevenidos pela pré-administração de benzodiazepínicos, fenotiazínicos, agonistas $\alpha_{2}$-adrenergicos, ou a administração concomitante de anestésicos inalatórios ( MUIR et al., 1992; VALADÃO, 2010).

Em relação aos efeitos respiratórios, a quetamina produz ventilação apnêustica e em altas doses a respiração pode ser irregular e superficial. É 
depressor respiratório moderado, mas em geral não afeta os gases sanguíneos. Entretanto em alguns pacientes, pode produzir hipóxia e hipercapnia marcante, especialmente quando utilizada em associação com fármacos depressores do SNC (SERTEYN et al., 1987; PERSON et al.,1999; LOREZAN et al., 2007; VALADÃO, 2010).

A quetamina quando utilizada em subdoses $\left(0,2\right.$ a $\left.0,5 \mathrm{mg} / \mathrm{kg}^{-1}\right)$, apresenta efeitos colaterais atenuados, entretanto mantendo sua ação analgésica devido sua ação nos receptores NMDA ( BARBIEREI et al., 1997) Slingsby e WatermanPearson (2000) demonstraram que a quetamina quando utilizada em subdoses (2,5 $\mathrm{mg} / \mathrm{Kg}$ ) previamente ao procedimento cirúrgico em pequenos animais promoveu analgesia eficiente mas de curta duração. Em outro estudo, Wagner et al. (2002) mostrou a eficiência da utilização de subdoses de quetamina administrada no período pré, trans e pós-operatório em cães submetidos à amputação.

É utilizada clinicamente em conjunto com sedativo-hipinótico, relaxante muscular e analgésico para produzir curta anestesia intravenosa ou para a indução da anestesia inalatória em cavalos (MUIR; SKADA; MILNE, 1977; LUNA TAYLOR; MASSONE, 1997). Ela também é administrada como adjuvante na anestesia geral para aumentar a profundidade anestésica e promover aumento no grau da analgesia (KUSHIRO et al., 2005). Possui propriedades analgésicas quando utilizada em subdose (CORRELL; MALEKI; GRACELY, 2004) e reduz o requerimento de halotano quando a concentração plasmática é maior de $1 \mu \mathrm{g} / \mathrm{ml}$ em cavalos sob anestesia geral, produzindo efeitos hemodinâmicos benéficos (MUIR; SAMS, 1992).

A quetamina, ainda tem sido utilizada na analgesia em estudos clínicos e experimentais, nas dores inflamatórias, neuropáticas e na dor pós-operatória, bem como na dor visceral (DAVIDSON; CARLTON, 1998; CHISTOPHER, 2000; BERRINO; OLIVA; MASSIMO, 2003).

\subsection{LIDOCAÍNA}

A Lidocaína é um anestésico local do grupo amida, o seu uso sistêmico tem mostrado efeito anestésico em muitos estudos experimentais em humanos (KOPPER; WEIGAND; NEUMANN, 2004) e em modelo de dor animal (ROBERTSON; SNCHEZ; MERRITT, 2005) 
Tradicionalmente a lidocaína tem sido usada com anestésico local e por via intravenosa para o tratamento de contração ventricular prematura. Em 1958 CliveLowe e colaboradores relataram o primeiro uso intravenoso da lidocaína como adjuvante da anestesia geral em seres humanos. A partir daí o seu uso tem sido documentada como parte da técnica da anestesia balanceada (KOPPERT; WEIGAND; NEUMANN, 2004).

Sua administração endovenosa age em terminações nervosas periféricas e centrais. Dados da literatura levam a crer que a sensibilização central resultante de lesão do tecido seria minimizada pela lidocaína por via venosa em diferentes níveis nervosos (periféricos ou centrais), dependendo do tecido lesado. Segundo (KAWAMATA; SUGINO; NARIMATSU, 2006), a incisão da pele de ratos resultou em diminuição do campo de recepção de estímulos periféricos nociceptivos e não diminuiu a resposta dos neurônios espinais em resposta a esse estímulo sugerindo uma ação anti-hiperalgésica periférica da lidocaína nesse modelo de dor. Já a dor neuropática aguda secundária ao trauma da medula espinal foi bloqueada pela administração de lidocaína por via venosa pela ação em canais de $\mathrm{Na}^{+}$e bloqueio da hiperexcitabilidade central (FINNERUP et al., 2005).

Quando a lidocaína é administrada por via intravenosa, ocorre aumento da concentração liquórica do neurotransmissor acetilcolina, que atuaria exacerbando as vias descendentes inibitória da dor, resultando em analgesia (ABELSON; HOGLUND, 2002). Provavelmente este fato ocorre devido a lidocaína se ligar no subtipo de receptor muscarínico $\mathrm{M} 3$, por meio da inibição de receptores para glicina e liberação de opióides endógenos, corroborando para o efeito analgésico final (CODA ; BAUSCH; HAAS, 1997; COHEN; MAO, 2003).

Além dos mecanismos de ação descritos, quando a lidocaína atinge a medula espinal reduz direta ou indiretamente a despolarização pós-sináptica mediada por receptores $N$-metil-D-aspartato e receptores para neurocininas (NAGY; WOOLF, 1996)

Por via endovenosa, a lidocaína diminui a resposta inflamatória à isquemia do tecido e atenua a lesão tecidual induzida por citocinas endoteliais e vasculares, por meio de mecanismos envolvendo a liberação de adenosina trifosfato e canal de $\mathrm{K}^{+}$ (DE KLAVER; BUCKINGHAM; RICH, 2003).

Em cavalos a lidocaína tem sido administrada, por infusão contínua, para promover analgesia, aprofundar a anestesia e com isto reduzir o requerimento do 
anestésico inalatório, além de aumentar a motilidade gastrointestinal no pósoperatório imediato (MUIR, 2009). A infusão contínua de lidocaína produz uma redução dose-dependente da CAM dos anestésicos inalatórios em pôneis, em cavalos (DOHERTY; FRAZIER, 1998) e em cães (VALVERDE et al., 2004) Pode também diminuir a incidência de íleo pós-operatório devido aos seus efeitos analgésico e antiinflamatório ( MUIR, 2009).

A lidocaína possui curta meia-vida, devido à sua alta metabolização hepática (ENGELKING; BLYDEN; LOFSTEDT, 1987). Para se alcançar níveis plasmáticos constantes em um curto espaço de tempo, primeiro deve ser administrada uma dose em bolus seguida de infusão contínua (DOHERTY; FRAZIER, 1998). Dzikiti 2003 em estudo com seis cavalos administrou o bolus de $2,5 \mathrm{mg} / \mathrm{kg}$, em 10 minutos, seguido de $50 \mu \mathrm{g} / \mathrm{kg} / \mathrm{min}$ em infusão contínua, obtendo um nível de concentração plasmática de 0,03 a $4,23 \mu \mathrm{g} / \mathrm{ml}$ com uma redução média da CAM do isoflurano em torno de $25 \%$ sem ser notados efeitos colaterais (DZIKITI ; HELLBREKERS; DIJK, 2003)

Também em estudo clínico foi investigado a influência da infusão contínua de lidocaína na recuperação da anestesia com isoflurano ou sevoflurano e foi mostrado que cavalos que receberam a infusão de lidocaína até o final da cirurgia tiveram um elevado grau de ataxia e baixa qualidade de recuperação. Embora este estudo não tenha correlação entre a concentração plasmática de lidocaína e a qualidade de recuperação, recomenda-se que a infusão contínua de lidocaína seja descontinuada 30 minutos antes do final da cirurgia com o objetivo de se reduzir possíveis ocorrências de ataxia durante o período de recuperação. (VALVERDE et al., 2005).

Apesar do peso corporal ser rotineiramente utilizado para a determinação da dose de anestésico local a ser administrada, não existe correlação entre o mesmo e a máxima concentração plasmática, tornando a dose calculada em $\mathrm{mg} / \mathrm{kg}^{-1}$ arbitrária. Entretanto, como a dose estipulada pelos fabricantes de anestésicos locais está aquém das doses tóxicas, trabalha-se em um patamar aparentemente seguro. A toxidade da lidocaína em humanos tem mais probabilidade de manifestar-se quando a concentração plasmática atinge $5 \mu \mathrm{g} / \mathrm{ml}^{1}$ (SUCENA; CACHAPUZ; LOMBARDIA, 2004). Doses consideradas pequenas variam de 1 a $2 \mathrm{mg} \mathrm{kg}^{-1}$, administradas em bolus, seguidas ou não de infusão contínua de $1,5 \mathrm{mg} \cdot \mathrm{kg}^{-1} \cdot \mathrm{h}^{-1}$, correspondendo à concentração plasmática de $2 \mu \mathrm{g} \cdot \mathrm{ml}^{-1}$ (ABELSON; HOGLUND, 2002). Porém, convém ficar atento para o fato de que as concentrações plasmáticas da lidocaína e seu metabólito ativo, a monoetilglicinexilidida, apresentam perfis farmacológicos 
diferentes quando administrados no animal anestesiado com isoflurano ou no animal acordado. O animal anestesiado apresenta volume do compartimento central, clearance e meia-vida de eliminação da lidocaína menores quando comparado com o animal acordado, resultando em maior concentração plasmática de lidocaína no animal anestesiado.(THOMASY; PYPENDOP; IIKIW, 2005). 


\section{JUSTIFICATIVA}

A anestesia balanceada promove analgesia perioperatória e uma melhora hemodinâmica, devido à diminuição do requerimento da concentração inalatória na anestesia geral.

Os opióides têm sido utilizados extensivamente no homem e em pequenos animais com excelentes resultados, porém, na espécie eqüina os estudos são escassos e pouco conclusivos.

Sendo assim, justificou-se o atual estudo, devido a necessidade de estudo clínico, que possa avaliar a condução do opióide em situação que haja estímulo doloroso real, trans e pós-operatório, fato que pode alterar a ação do fármaco. 


\section{OBJETIVOS}

Este experimento teve como objetivo determinar, se o fentanil em infusão contínua, seria capaz de diminuir a fração expirada de isoflurano, promovendo uma melhor recuperação após cirurgia de artroscopia, em comparação à infusão de lidocaína ou quetamina, durante a manutenção da anestesia com isoflurano. 


\section{MATERIAL E MÉTODO}

Segue a descrição dos animais utilizados neste estudo, o preparo dos mesmos, o procedimento anestésico executado, a descrição do procedimento experimental, as avaliações paramétricas efetuadas e metodologia da estatística empregada.

\subsection{LOCAL DE REALIZAÇÃO DO ESTUDO}

O presente estudo foi realizado no Hospital Veterinário da Faculdade de Medicina Veterinária e Zootecnia da Universidade de São Paulo. O protocolo experimental foi aprovado pela Comissão de Bioética da referida Instituição.

\subsection{ANIMAIS}

Foram utilizados 20 animais da espécie eqüina, machos e fêmeas, com idade variada de 3 a 8 anos de variadas raças, com peso entre 350 a 500 quilos, proveniente do serviço de cirurgia do Hospital Veterinário da FMVZ-USP, para serem submetidos à cirurgia de artroscopia.

\subsection{CRITÉRIO DE INCLUSÃO}

Nesse estudo foram incluídos cavalos saudáveis, os quais foram avaliados através de anamnese, exame físico e análise laboratorial, estando os mesmos sob jejum alimentar por 12 horas, sendo excluídos aqueles portadores de qualquer anomalia que pudesse interferir no andamento do estudo.

\subsection{PROTOCOLO EXPERIMENTAL}

A veia jugular externa, após tricotomizada, foi cateterizada com cateter clibre $14 \mathrm{G}^{1}$. A técnica anestésica empregada constou da administração de detomidina ${ }^{2}$

\footnotetext{
1 Jelco - Medex Medical LTDA, Inglaterra
}

2 Dormiun v - Agener União - São Paulo 
$(10 \mu \mathrm{g} / \mathrm{kg})$ pela via intravenosa, como medicação pré-anestésica (MPA) em todos os animais e após 10 minutos da MPA, a indução foi realizada por via intravenosa, com quetamina $^{3}(2 \mathrm{mg} / \mathrm{kg})$ associado a diazepam ${ }^{4}(0,05 \mathrm{mg} / \mathrm{kg})$. Após o decúbito foi administrado uma solução a $10 \%$ de Éter Gliceril Guaiacol ${ }^{5}(100 \mathrm{mg} / \mathrm{kg})$ para promover um adequado relaxamento muscular para posterior intubação orotraqueal.

Os animais foram intubados com sonda traqueal ${ }^{6}$ de diâmetro apropriado, sendo a anestesia mantida com $70 \%$ de $\mathrm{O}_{2}$ e isoflurano na fração expirada de 1,4 1,6 , mensurada por meio de analisador de gases e mantido em concentração suficiente para obtenção do plano anestésico adequado para a realização do procedimento cirúrgico.

Após a indução os animais foram posicionados em decúbito dorsal na mesa cirúrgica e submetidos à ventilação mecânica na modalidade de volume controlado (VVC) em aparelho anestesia com ventilador microprocessado para grandes animais ${ }^{7}$. Os parâmetros ventilatórios iniciais constaram de volume corrente de $14 \mathrm{ml} / \mathrm{kg}$, frequência respiratória de 10 movimentos por minuto, relação $\mathrm{I} / \mathrm{E} 1: 3$ e $\mathrm{FiO}_{2}$ de $70 \%$, além de PEEP $7 \mathrm{cmH}_{2} \mathrm{O}$. Foram mensuradas continuamente a pressão parcial de dióxido de carbono no ar expirado $\left(\mathrm{EtCO}_{2}\right)$, com auxílio de capnógrafo do analisador de gases ${ }^{8}$ acoplado à sonda traqueal, a saturação periférica de hemoglobina por meio de oximetria de pulso ${ }^{9}$. $\mathrm{E} \mathrm{EtCO}_{2}$ foi mantida entre 45 e 55 $\mathrm{mmHg}$, com o aumento ou diminuição inicialmente do volume corrente e ou da frequência respiratória $(\mathrm{FR})$ quando os limites não foram suficientes para corrigir uma eventual hipocapnia ou hipercapnia.

Um cateter $22 \mathrm{G}^{1}$ foi introduzido na artéria facial para manutenção das pressões arteriais e para coleta das amostras hemogasométricas. A gasometria arterial foi analisada por meio de monitor de bancada ${ }^{10}$. Durante o procedimento cirúrgico os animais receberam fluidoterapia com Ringer lactato ${ }^{11}$ na taxa de 10 $\mathrm{ml} . \mathrm{kg}^{-1} \cdot \mathrm{hora}^{-1}$.

\footnotetext{
${ }^{3}$ Ketamina - Cloridrato de Cetamina- Agener União - São Paulo.

${ }^{4}$ Diazepam - Cristália Produtos Químicos Farmacêuticos Ltda., São Paulo, Brasil

${ }^{5}$ EGG - Eter Gliceril Guaiacol - Tianjin Xinxin - China..........

${ }^{6}$ Surgivet- Smiths Medical PM, Inc.

7 Linea-LA- Anesthesia Large Animal - Intermed.

${ }^{8}$ Criticare- POET IQ2 - Analisador de gases anestésicos e capnógrafo

9 Efedrinß - Sulfato de efedrina - Cristália Produtos Químicos Farmacêuticos Ltda., São Paulo, Brasil

${ }^{10}$ ABL5 - Radiometer, Copenhagen, Dinamarca.

${ }^{11}$ Solução de Ringer Lactato - JP Indústria Farmacêutica S.A
} 
A hipotensão foi considerada quando a pressão arterial média atingiu valores abaixo de $60 \mathrm{mmHg}$. Neste caso, foi utilizada a efedrina ${ }^{9}$ titulada na concentração de $0,15 \mathrm{mg} / \mathrm{ml}$ até a normalização deste parâmetro.

\subsection{DELINEAMENTO EXPERIMENTAL}

Os animais foram divididos aleatoriamente em 4 grupos com 5 animais em cada:

> Grupo Isoflurano ( $\mathrm{Gl}, \mathrm{n}=5)$ : os animais deste grupo não receberam nenhum tratamento adjuvante ao isoflurano, sendo apenas anestesiados com este anestésico inalatório e submetidos à cirurgia de artroscopia.

> Grupo Fentanil (GF, n=5): os animais receberam $7 \mu \mathrm{g} / \mathrm{Kg}$ de fentanil em bolus durante 15 minutos, seguido de infusão contínua de $0,7 \mu \mathrm{g} / \mathrm{Kg} /$ minuto em bomba de infusão ${ }^{12}$, como adjuvante do anestésico inalatório, isoflurano durante a cirurgia de artroscopia.

> Grupo Lidocaína (GL, $\mathrm{n}=5$ ): os animais receberam 1,5mg/kg/ de lidocaína em bolus durante 15 minutos, seguido de infusão contínua de $0,15 \mathrm{mg} / \mathrm{kg} / \mathrm{minuto}$ em bomba de infusão ${ }^{12}$, como adjuvante do anestésico inalatório, isoflurano durante a cirurgia de artroscopia.

Grupo Quetamina ( $G Q, n=5)$ : os animais receberam $2 \mathrm{mg} / \mathrm{kg} /$ de quetamina em bolus durante 15 minutos, seguido de infusão contínua de $0,2 \mathrm{mg} / \mathrm{kg} /$ minuto em bomba de infusão ${ }^{12}$, como adjuvante do anestésico inalatório, isoflurano durante a cirurgia de artroscopia.

\subsection{DELINEAMENTO TEMPORAL}

Decorridos 20 minutos da instrumentação e estabilização da anestesia, as avaliações hemodinâmicas, de mecânica respiratória e de parâmetros hemogasométricos foram realizadas em seis momentos:

20INAL: após 20 minutos do início da anestesia inalatória;

APBOLUS: imediatamente após bolus;

15BOLUS: 15 minutos após bolus;

\footnotetext{
${ }^{12}$ Lifecare Plum XLD - Hospira Produtos Hospitalares LTDA - São Paulo
} 
30BOLUS: 30 minutos após bolus;

45BOLUS: 45 minutos após o bolus;

75BOLUS: 75 minutos após bolus.

Final da infusão 60min

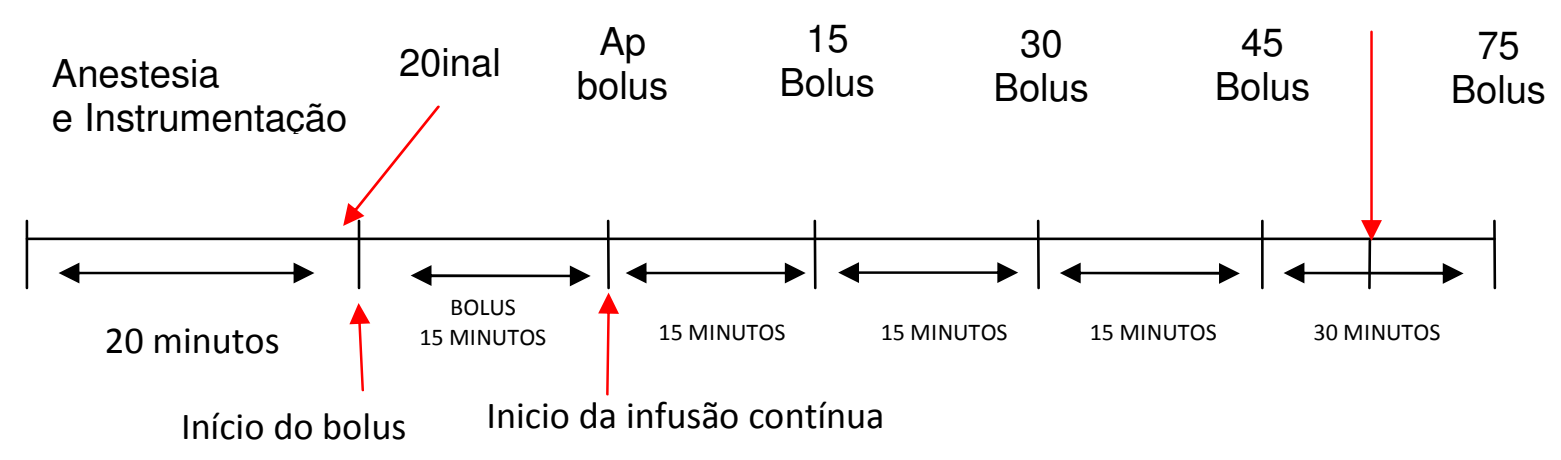

\subsection{PARÂMETROS ANALISADOS}

Durante o procedimento anestésico-cirúrgico, foram avaliados e mensurados os parâmetros descritos a seguir.

\subsubsection{Variáveis Hemodinâmicas}

\section{Frequência e Ritmo Cardíaco}

Durante o decorrer da anestesia, a freqüência cardíaca e o ritmo cardíaco foram avaliados por meio de cardioscópio do monitor multiparamétrico ${ }^{13}$.

\section{Pressão Arterial Sistêmica}

A pressão foi obtida por mensuração direta através do cateter da artéria facial acoplado ao transdutor de pressão do monitor de pressão, obtendo-se desta forma a pressão arterial média (PAM), sistólica (PAS) e diastólica (PAD)

\subsubsection{Variáveis de Oxigenação, de ventilação e da mecânica pulmonar}

\footnotetext{
${ }^{13}$ Viridia - CMS - HP 66S - Germany
} 


\section{Frequência Respiratória}

Durante o decorrer da anestesia, a freqüência respiratória foi monitorada por meio do monitor de mecânica respiratória ${ }^{14}$.

Pressão Parcial de Oxigênio $\left(\mathrm{PaO}_{2}\right)_{2}$, Pressão Parcial de Dióxido de Carbono $\left(\mathrm{PaCO}_{2}\right)$ e Saturação de Oxigênio no Sangue Arterial $\left(\mathrm{SaO}_{2}\right)$

A coleta de sangue arterial para obtenção dos valores $\mathrm{PaO}_{2}, \mathrm{PaCO}_{2}$ e $\mathrm{SaO}_{2}$ foi realizada através do cateter na artéria facial.

As amostras foram coletadas em seringa heparinizadas sendo a agulha vedada com tampa de borracha, evitando-se assim o contato do sangue com o ar ambiente. $\mathrm{O}$ exame de cada amostra foi realizado em analisador de $\mathrm{pH}$ e gases sanguíneos ${ }^{12}$.

\section{$\underline{\text { Pressão de dióxido de carbono no final da expiração }\left(\mathrm{ETCO}_{2}\right)}$}

A pressão de dióxido de carbono no final da expiração foi avaliada através do capnógrafo ${ }^{8}$ acoplado entre o tubo endotraqueal e a traquéia corrugada do aparelho de anestesia.

\section{Volume Inspiratório, Volume Expiratório, Pressão de Platô, Peep e Pressão de Pico}

Os volumes de ar inspirado, expirado, pressão de platô, pressão positiva do final da expiração e pressão de pico foram mensurados através do monitor de mecânica respiratória do aparelho de anestesia de grandes animais ${ }^{7}$.

\section{Fração expirada de isoflurano [ISO](Fexp\%)}

A fração expirada de isoflurano foi avaliada através do capnógrafo e analisador de gases ${ }^{8}$ acoplado entre o tubo endotraqueal e a traquéia corrugada do aparelho de anestesia.

\footnotetext{
${ }^{14}$ Win tracer 3,0 - Intermed Prod. Med. Hosp. São Paulo- S.P
} 
Complacência estática (Cest) e Resistência de vias aéreas (Rva)

A complacência estática e resistência de vias aéreas foram calculadas através das fórmulas:

Complacência estática:

$$
\text { Cest }=\text { Vex } / \text { Plat }- \text { PEEP }
$$

Onde: $\quad$ Vex $=$ Volume expiratório

Plat $=$ Platô

PEEP $=$ Pressão positiva no final da expiração

$\underline{\text { Resistência das vias aéreas: }}$

$$
\begin{aligned}
& \text { Rva }=\text { Pico }- \text { Plat } / \text { Fluxo } \\
& \text { Fluxo }=\text { Vins } / \text { Tinsp } \\
& \text { Tinsp }=60 / F R / 4
\end{aligned}
$$

Onde: $\quad$ Vins $=$ volume inspiratório

Tinsp $=$ Tempo inspiratório

\subsubsection{Avaliação Metabólica}

Bicarbonato Plasmático $\left(\mathrm{HCO}_{3}\right)$, Déficit de Base $(\mathrm{BE})$ e $\mathrm{pH}$ do Sangue Arterial

As mesmas amostras de sangue obtidos pelo cateter posicionados na artéria facial foi utilizado para a mensuração do bicarbonato plasmático, déficit de base e $\mathrm{pH}$, sendo realizados no mesmo analisador de gases sanguíneo e $\mathrm{pH}$ empregado para análise das demais variáveis.

\section{Temperatura Corpórea}

A temperatura corpórea foi obtida por meio de termômetro clínico em contato com a mucosa retal e mensurada em graus Celsius. 
Débito Urinário

O débito urinário foi mensurado por meio de cateterização vesical transuretral, levando-se em conta o volume obtido ( $\mathrm{ml}$ ) ao término do período de avaliação.

\subsection{ESCORE DE QUALIDADE DE RECUPERAÇÃO}

Estes parâmetros foram avaliados segundo a escala de avaliação da qualidade de recuperação, de Donaldson et al. (2000) (Quadro 1).

\subsection{ANÁLISES ESTATÍSTICA}

Os valores obtidos foram confrontados estatisticamente através de provas paramétricas, por meio de (ANOVA), empregando-se análise de variância para medidas repetidas seguida do teste de Tukey para a comparação dos diferentes momentos de observação de um mesmo grupo. Para a análise entre os grupos experimentais utilizou-se o teste T-Student. O grau de significância estabelecido para análise estatística foi de $5 \%(p<0,05)$. Os testes estatísticos foram realizados em programa de computados Instat ${ }^{15}$.

${ }^{15}$ Instat- Graphpad Software 


\begin{tabular}{|c|c|c|c|}
\hline $\begin{array}{l}\text { Fase de recuperação pós- } \\
\text { cirúrgica }\end{array}$ & Qualidade & Pontuação & Tempo \\
\hline I. Atitude geral & $\begin{array}{l}\text { 1-calmo } \\
\text { 3- calmo/determinado } \\
\text { 5- ansioso } \\
\text { 7- confuso, desnorteado } \\
\text { 8- nervoso } \\
\text { 10- frenético }\end{array}$ & & \\
\hline II. Decúbito Lateral & $\begin{array}{l}\text { 1- tranqüilo, esforço ocasional } \\
\text { 3- tenso } \\
\text { 5- debatendo-se }\end{array}$ & & \\
\hline III. Decúbito Esternal & $\begin{array}{l}\text { 1- estável, metódico } \\
\text { 5- tempestuoso, mas controlado } \\
\text { 10- debatendo-se com quedas }\end{array}$ & & \\
\hline \multicolumn{4}{|l|}{$\begin{array}{l}\text { IV. Tentativas para } \\
\text { Esternal }\end{array}$} \\
\hline V. Fase Esternal & $\begin{array}{l}\text { 1- uma pausa organizada } \\
\text { 3- inexistente } \\
\text { 6- prolongado } \\
\text { 7- múltiplos } \\
\text { 10- debatendo-se }\end{array}$ & & \\
\hline VI. Posição Quadrupedal & $\begin{array}{l}\text { 1- metódico } \\
\text { 3-difícil } \\
\text { 6- apoiando-se nas paredes } \\
\text { 10- debatendo-se nas paredes }\end{array}$ & & \\
\hline VII. Força /Resistência & $\begin{array}{l}\text { 1- próximo do máximo } \\
\text { 3- intermediário } \\
\text { 6- caiu antes de erguer-se }\end{array}$ & & \\
\hline \multicolumn{4}{|l|}{$\begin{array}{l}\text { VIII. Tentativas para } \\
\text { Estação }\end{array}$} \\
\hline $\begin{array}{l}\text { IX. Equilíbrio e } \\
\text { Coordenação }\end{array}$ & $\begin{array}{l}\text { 1- sólido } \\
\text { 3- cambaleante } \\
\text { 5- manutenção dos reflexos } \\
\text { 8- hesitante } \\
\text { 10- repetidas quedas }\end{array}$ & & \\
\hline X. Flexão das Falanges & $\begin{array}{l}\text { 1- ausente } \\
\text { 2- membros pélvicos - suave } \\
\text { 3- membros pélvicos - marcado } \\
\text { 4- quatro membros- moderado } \\
\text { 5- excessivo, prolongado }\end{array}$ & & \\
\hline
\end{tabular}

Donaldson LL, Dunlop GS, Holland MS, Burton BA. The recovery of horses from inhalant anesthesia: a comparison of halothane and isoflurano. Veterinary Surgery, 2000, 29(1): 92-101

Quadro-1 Escala de avaliação da qualidade de recuperação, Donaldson et al. (2000) 


\section{RESULTADOS}

A seguir há descrição dos resultados obtidos. Os valores individuais, média e desvio-padrão dos parâmetros obtidos de todos os grupos estão descritos nos apêndices de $A$ até $D$.

\subsection{PARÂMETROS HEMODINÂMICOS DIRETOS}

\section{Análise Entre Momentos}

Freqüência cardíaca (FC)

A freqüência cardíaca manteve-se estável não apresentando diferença significativa entre os grupos estudados (Gráfico 1 e Tabela 1 ).

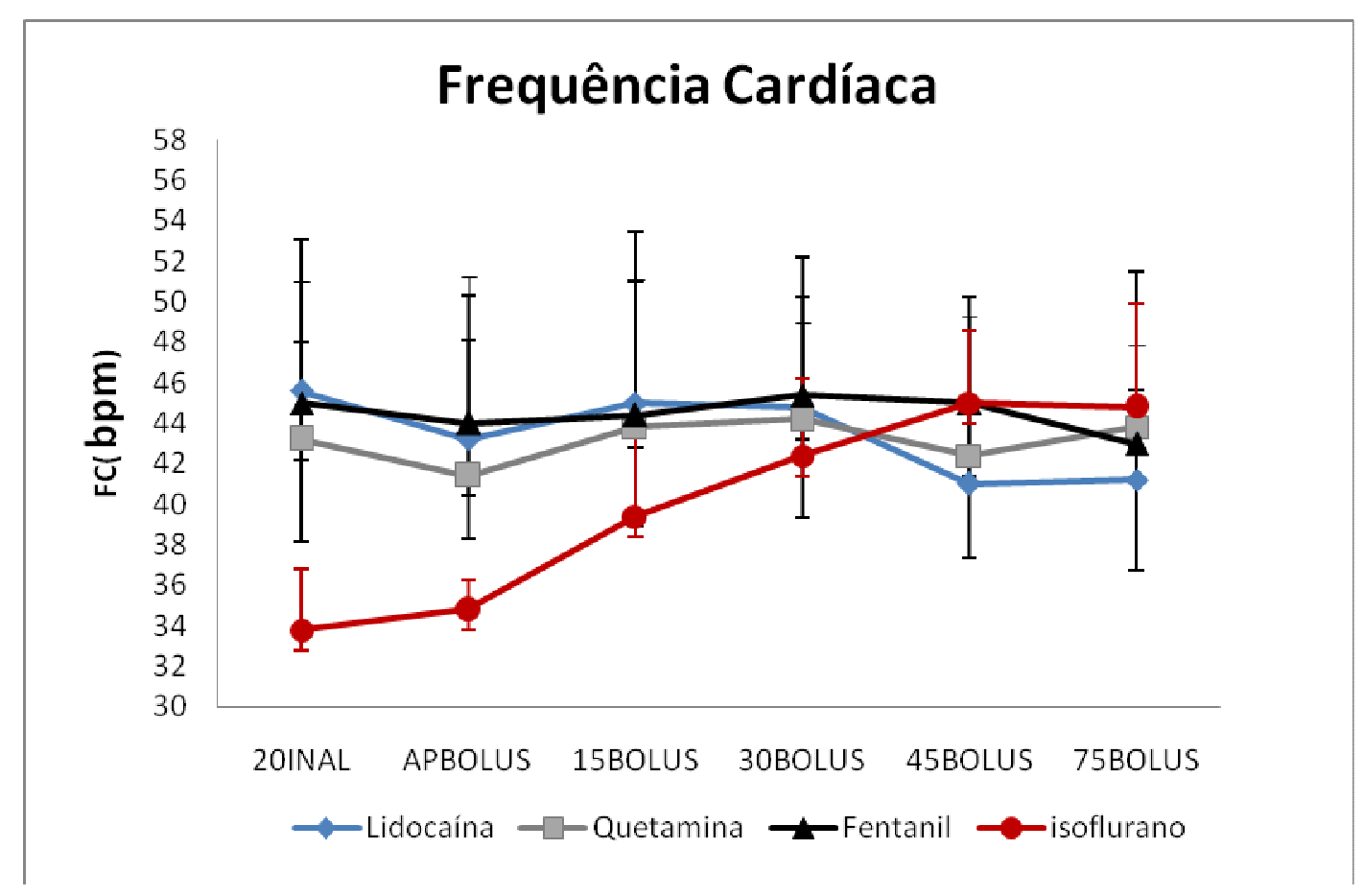

Gráfico 1 - variação da frequência cardíaca (média e desvio padrão) em equinos dos grupos GL (lidocaína); GQ (quetamina); GF (fentanil) - São Paulo - 2009 


\section{Pressão arterial sistólica (PAS)}

A pressão arterial sistólica no grupo quetamina (GQ) apresentou aumento significativo no momento 75 Bolus $(101,0 \pm 17,26 \mathrm{mmHg})$ com relação ao momento 20 Inal $(66,6 \pm 11,5 \mathrm{mmHg})$ com $p<0,01$. No grupo Isoflurano $\mathrm{Gl}$ no momento 75 Bolus a pressão arterial sistólica apresentou um aumento significativo $(66,3 \pm 20,17 \mathrm{mmHg})$ quando confrontado com o momento 20 Inal $(51,50 \pm 9,33)$ sendo $p<0,05$. Nos demais grupos não houve alterações significativas (Gráfico 2 e Tabela 1).

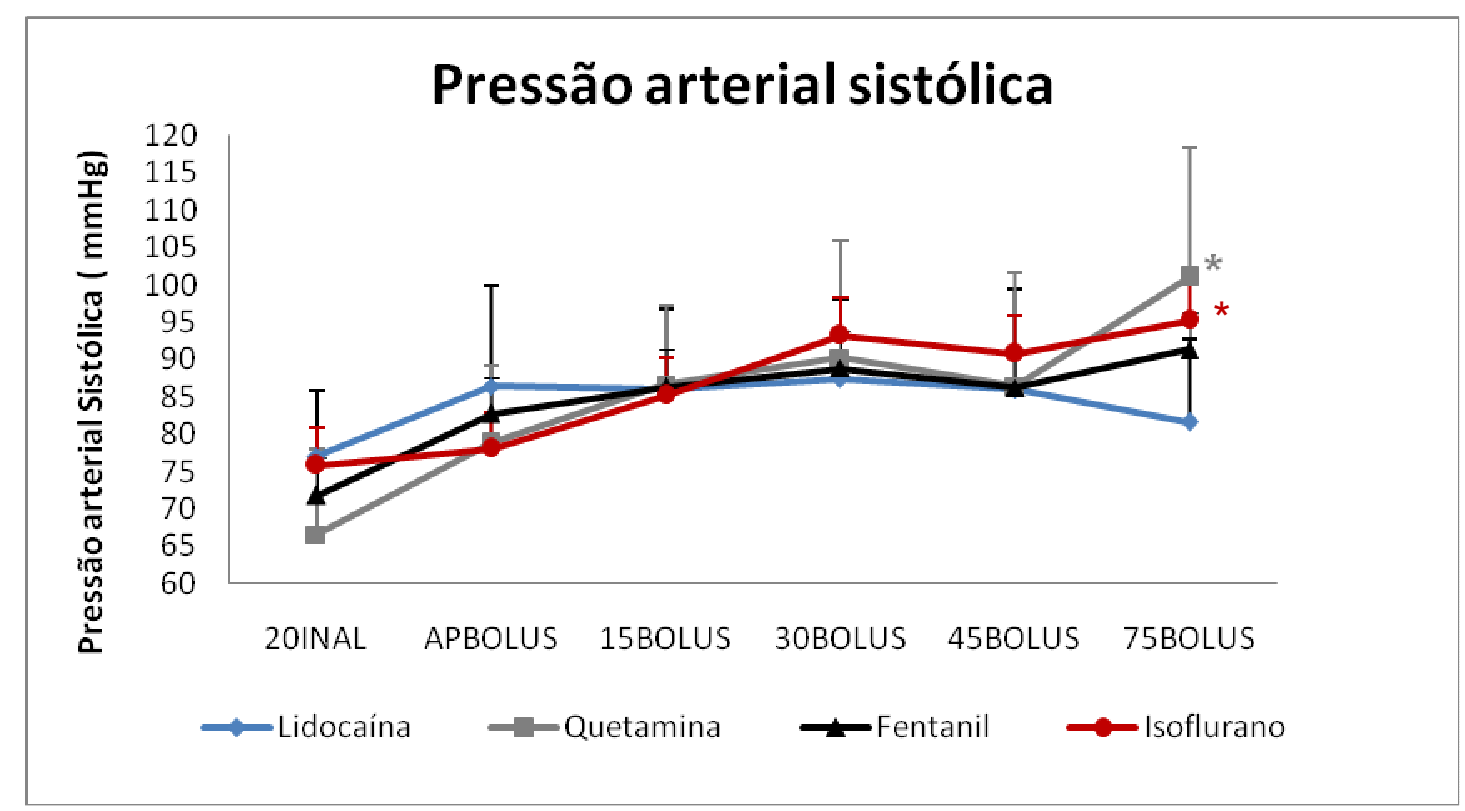

Gráfico 2 - Variação da pressão arterial sistólica ( média e desvio padrão) em equinos dos grupos: Lidocaína (GL); Quetamina(GQ); Fentanil (GF) e Isoflurano (Gl); *diferiu do momento 20inal

\section{Pressão arterial média (PAM)}

A pressão arterial média do grupo quetamina (GQ) apresentou aumento significativo no momento 75 Bolus $(84,4 \pm 18,9 \mathrm{mmHg})$, com em relação ao momento 20 Inal $(32,2 \pm 8,98 \mathrm{mmHg})$ com $p<0,01$. No grupo isoflurano (Gl) também houve aumento significativo deste parâmetro no momento 75Bolus $(75,8 \pm 14,1 \mathrm{mmHg})$, quando confrontado com os momentos 20INAL $(52,4 \pm 4,8 \mathrm{mmHg})$ com $p<0,01 \mathrm{e}$ APBOLUS $(57,8 \pm 2,9 \mathrm{mmHg})$ com $p<0,05$ (Gráfico 3 e Tabela 1) 


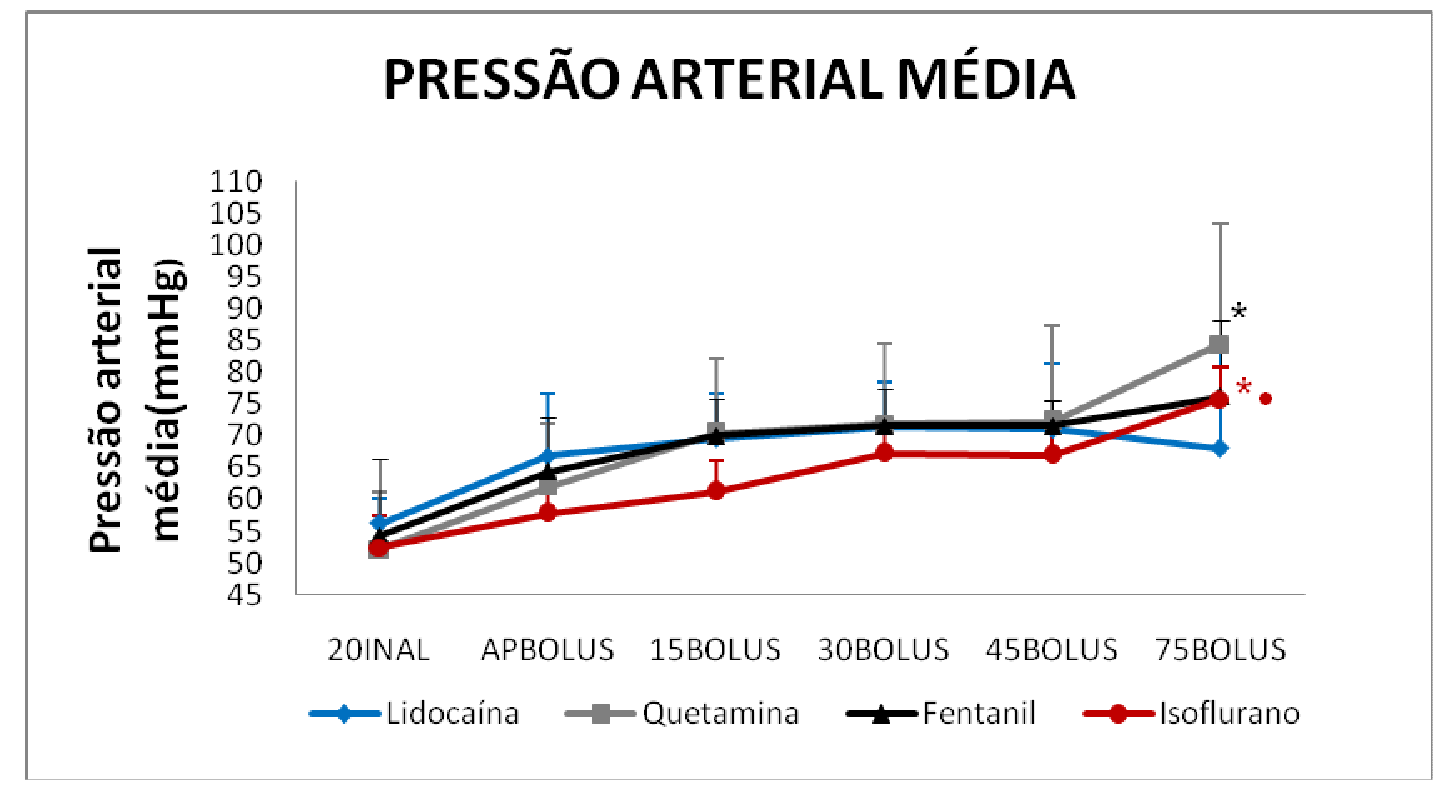

Gráfico 3 - Variação da pressão arterial média ( média e desvio padrão) em equinos dos grupos: Lidocaína (GL); Quetamina(GQ); Fentanil (GF) e Isoflurano (GI); *diferiu do momento 20inal $(p<0,01)$; diferiu do momento APbolus $(p<0,05)$

\section{Pressão arterial diastólica (PAD)}

A pressão arterial diastólica no grupo quetamina (GQ) apresentou aumento significativo estudo 75Bolus $(73,0 \pm 19,1 \mathrm{mmHg})$ em relação a 20 minutos após o início da anestesia inalatória $(43,8 \pm 9,1 \mathrm{mmHg})$ com $p<0,01$. No grupo isoflurano (Gl) houve aumento da pressão arterial diastólica (PAD) no momento 75Bolus $(63,2 \pm 11,67 \mathrm{mmHg})$ diferindo dos momentos $20 \mathrm{Inal}(42,6 \pm 6,4 \mathrm{mmHg})$ com $p<0,001$, imediatamente após o bolus ApBolus $(46,0 \pm 2,45 \mathrm{mmHg})$ sendo $p<0,01$ e 15Bolus $(48,6 \pm 5,8 \mathrm{mmHg}) \mathrm{p}<0,05$ (Gráfico 4 e Tabela 1).

\section{Análise Entre Grupos}

Não foram observadas diferenças estatísticas na frequência cardíaca entre os grupos estudados, bem como na pressão arterial sistólica (PAS) e pressão arterial média (PAM). 
Pressão arterial Diastólica (PAD)

O grupo isoflurano demonstrou PAD significativamente maior $(63,2 \pm 11,67 \mathrm{mmHg})$, que o grupo fentanil $(49,4 \pm 9,66 \mathrm{mmHg})$ no momento $75 \mathrm{BOLUS}$ com $p<0,05$. O grupo quetamina apresentou PAD maior $(73,0 \pm 19,10 \mathrm{mmHg})$ que 0 grupo fentanil $(49,4 \pm 9,66 \mathrm{mmHg})$ com $p<0,05$, também no momento 75BOLUS (Gráfico 4 e Tabela 1).

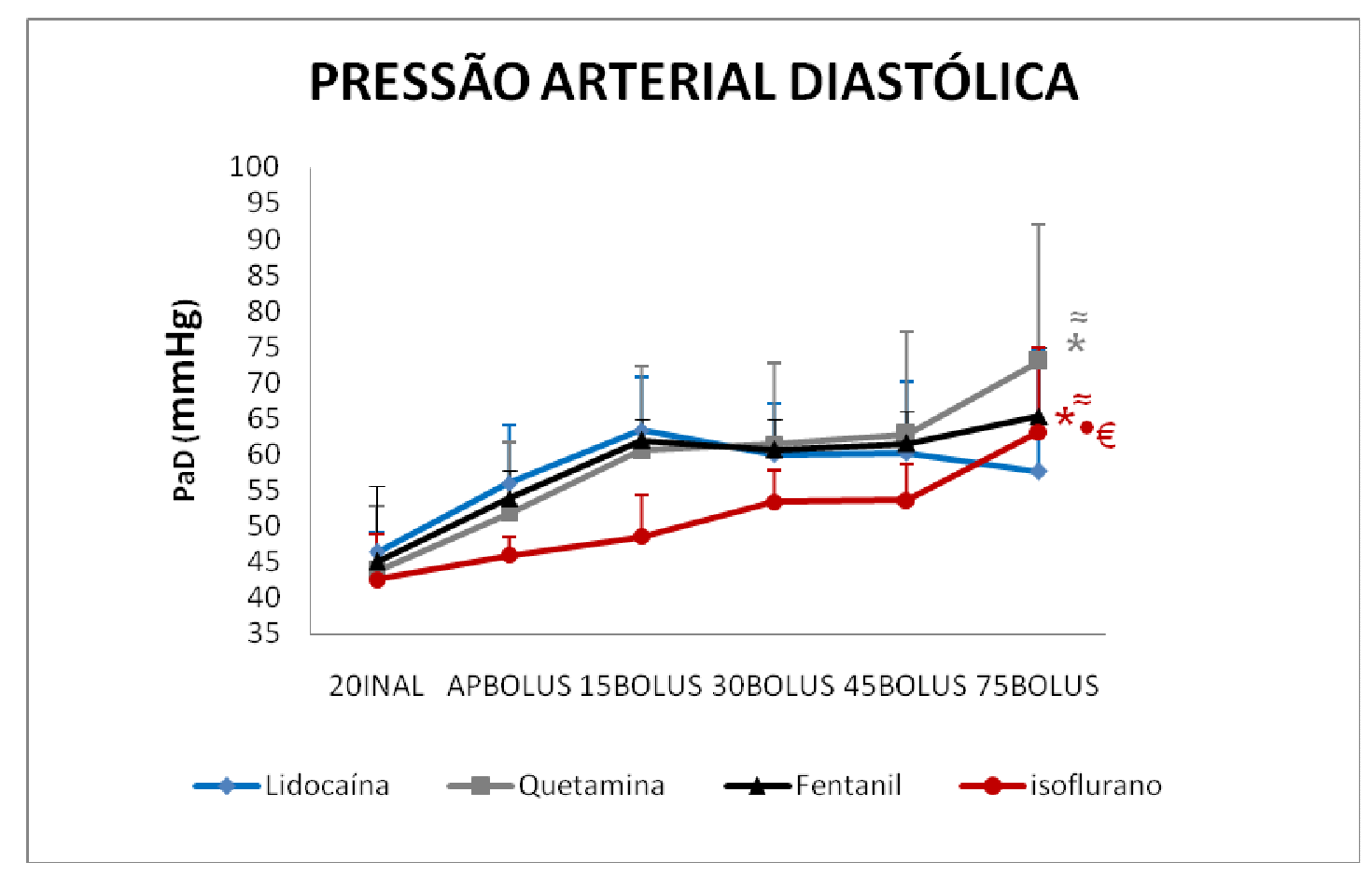

Gráfico 4 - Variação da pressão arterial diastólica ( média e desvio padrão) em equinos dos grupos: Lidocaína (GL); Quetamina(GQ); Fentanil (GF) e Isoflurano (GI); * diferiu estatisticamente do momento 20INAL; • diferiu estatisticamente do momento APBOLUS; $€$ diferiu estatisticamente do momento 15BOLUS; diferiu significativamente do grupo fentanil (GF) 


\subsection{PARÂMETROS RELACIONADOS À OXIGENAÇÃO E FRAÇÃO EXPIRADA DE ISOFLURANO}

\section{Análise Entre Momentos}

Não houve alteração significativa entre os momentos e entre grupos nos parâmetros pressão parcial de oxigênio no sangue arterial $\left(\mathrm{PaO}_{2}\right)$ e na saturação de oxigênio no sangue arterial $\left(\mathrm{SaO}_{2}\right)$.

\section{Fração expirada de Isoflurano}

Em relação à fração expirada de isoflurano, observou-se no grupo GL uma diminuição significativa nos momentos 15Bolus $(1,00 \pm 0,08 \%)$ com $p<0,01,30$ minutos após bolus $(0,90 \pm 0,05 \%)$ sendo $p<0,001$ e 45 minutos após o bolus( $0,90 \pm 0,08 \%), p<0,001$ com uma queda de $33 \%, 40 \%$ e $40 \%$ respectivamente em ralação ao momento $20 \operatorname{INAL}(1,50 \pm 0,18 \%)$. Ainda foi observado uma queda de $15 \%$, no momento 45 Bolus $(0,90 \pm 0,08 \%)$ quando confrontado com o momento imediatamente após bolus (APBOLUS) $(1,20 \pm 0,13 \%)$ com $p<0,05$.

No grupo quetamina $(\mathrm{GQ})$ houve uma diminuição significativa nos momento $15(1,04 \pm 0,29 \%)$ e 30 bolus $(0,96 \pm 0,15 \%) \quad p<0,05$ em relação ao momento 20INAL $(1,44 \pm 0,11 \%)$ com uma queda de $28 \%$ e $33 \%$ respectivamente.

Observou-se que no grupo GF houve uma diminuição significativa nos momentos $15(1,04 \pm 0,11 \%), 30(0,96 \pm 0,05 \%)$ e 45 Bolus( $1,00 \pm 0,00 \%)$ com quedas de $21 \%, 27 \%$ e $24 \%$ em relação ao momento 20 minutos após o início da anestesia inalatória( $1,32 \pm 0,04 \%)$, sendo que a maior queda foi no momento 30 Bolus. Neste mesmo grupo também houve queda significativa da fração expirada de isoflurano de $20 \%$ entre o momento APBOLUS $(1,20 \pm 0,12 \%)$ quando comparado ao momento 30 Bolus $(0,96 \pm 0,05 \%)$ sendo $p<0,01$ e queda de $17 \%$ em 45 Bolus $(1,00 \pm 0,00 \%)$ quando confrontado com o momento imediatamente após bolus (APBOLUS) $(1,20 \pm 0,12 \%) p<0,05$.

Foi observado ainda no grupo GF aumento significativo da fração expirada de isoflurano no final do estudo (75BOLUS) $(1,38 \pm 0,15 \%)$ em relação aos momentos 15 $(1,04 \pm 0,11 \%) 30(0,96 \pm 0,05 \%)$ e 45 Bolus $(1,00 \pm 0,00 \%), p<0,001$. 
Não foram observadas variações entre momentos em relação à fração expirada de isoflurano no grupo $\mathrm{Gl}$, mantendo-se alta em todos os momentos.

\section{Análise Entre Grupos}

\section{Fração expirada de Isoflurano}

Em relação à fração expirada de isoflurano observou-se uma queda significativa de $37 \%$ no GL $(1,00 \pm 0,08 \%) p<0,001,35 \%$ no GQ $(1,04 \pm 0,29 \%)$ sendo $p<0,01$ e $35 \%$ no GF $(1,04 \pm 0,11 \%)$ com $p<0,001$, no momento 15 Bolus quando comparada ao grupo $\mathrm{Gl}$.

A partir do momento 30Bolus observou-se uma queda de $47 \%$ no grupo $\mathrm{GL}$ $(0,90 \pm 0,05 \%)$ com $p<0,001$, e $44 \%$ nos grupos $G Q(0,96 \pm 0,15 \%)$ sendo $p<0,001$ e $44 \% \mathrm{GF}(0,96 \pm 0,05 \%)$ com $\mathrm{p}<0,001$ em relação ao grupo $\mathrm{Gl}$.

Já no momento 45Bolus a queda foi de $44 \%$ no grupo GL $(0,90 \pm 0,08 \%) p<0$, $001,34 \%$ no grupo GQ $(1,06 \pm 0,19 \%)$ sendo $p<0,01$ e $38 \%$ no grupo GF $(1,00 \pm 0,00 \%) p<0,001$ em relação ao grupo $\mathrm{Gl}$.

No momento 75BOLUS a queda observada foi de $30 \%$ no grupo GL $(1,20 \pm 0,29 \%)$ com $p<0,001,36 \%$ no grupo $G Q(1,10 \pm 0,19 \%), p<0,001$ e $19 \%$ no

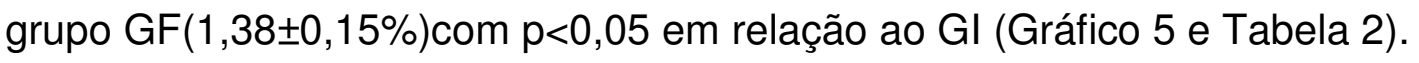




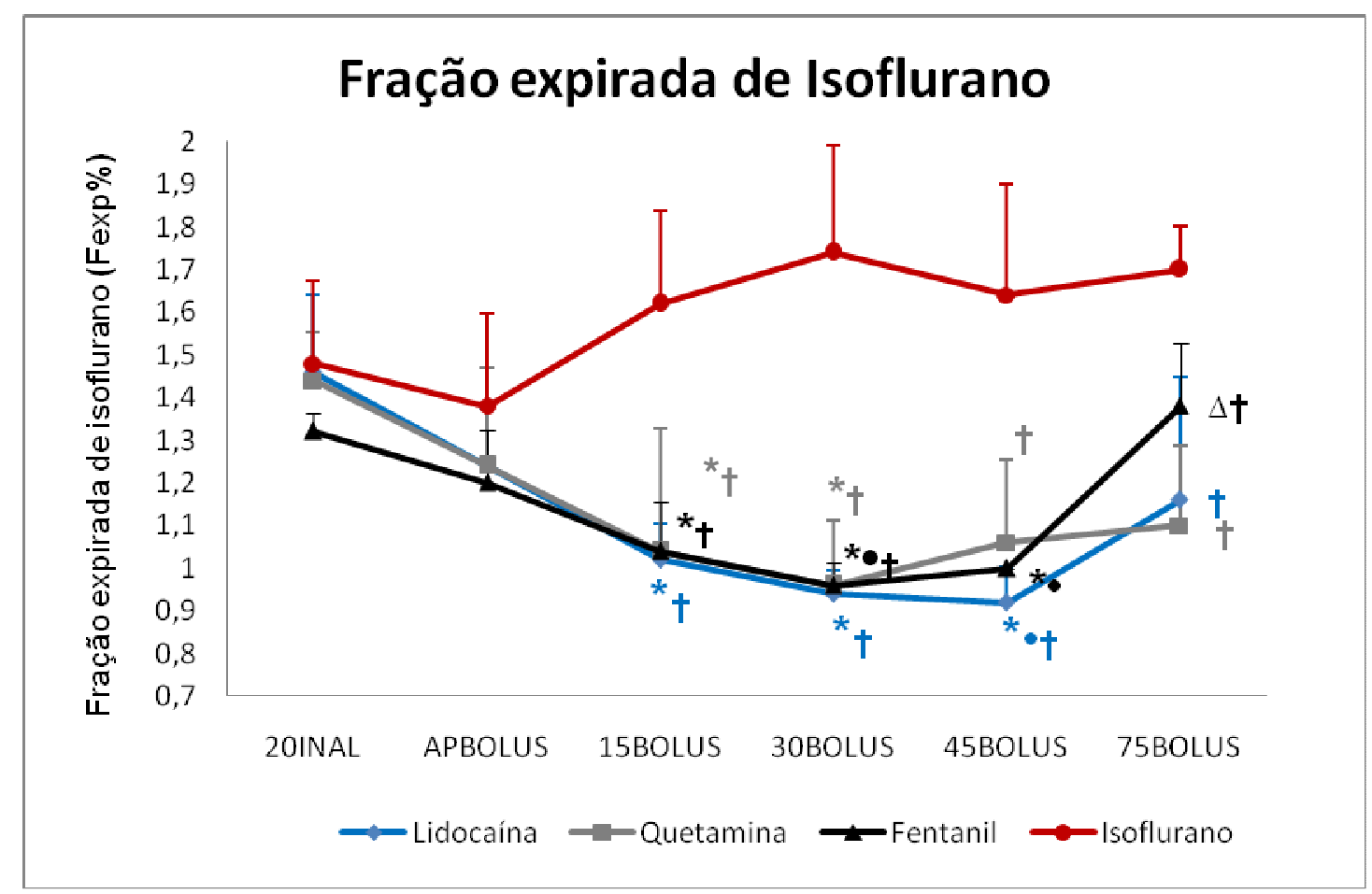

Gráfico 5- Variação da fração expirada de isoflurano ( média e desvio padrão) em equinos dos grupos: Lidocaína (GL); Quetamina(GQ); Fentanil (GF) e Isoflurano (GI); * diferiu estatisticamente do momento 20INAL; • deferiu estatisticamente do momento APBOLUS; $\Delta$ diferiu estatisticamente dos momentos 15,30 e 45BOLUS; † diferiu estatisticamente do grupo isoflurano (GI)

\subsection{CONSUMO DE FLUIDO, DE EFEDRINA E DIURESE EM 1HORA DE PROCEDIMENTO}

\section{Análise Entre grupos}

O grupo Lidocaína (GL) apresentou um consumo médio de 14,10 $\mathrm{ml} / \mathrm{Kg}$ de Ringer com Lactato de Sódio; 43,5 mg de efedrina e teve uma diurese média de 5,2 $\mathrm{ml} / \mathrm{Kg}$ (Gráficos 6,7 e 8 e Quadro 2).

O grupo quetamina (GQ) apresentou um consumo médio de $11,44 \mathrm{ml} / \mathrm{Kg}$ de Ringer com Lactato de Sódio; 96,2 mg de efedrina e uma diurese média de $2 \mathrm{ml} / \mathrm{kg}$ (Gráficos 6, 7 e 8 e Quadro 2). 
O grupo fentanil (GF) apresentou um consumo médio de $13,13 \mathrm{ml} / \mathrm{kg}$ de Ringer com Lactato de Sódio; $84 \mathrm{mg}$ de efedrina e uma diurese média de 6,5 ml/kg (Gráficos 6,7 e 8 e Quadro 2).

O grupo isoflurano (GI) apresentou um consumo médio de $17,74 \mathrm{ml} / \mathrm{Kg}$ de Ringer com Lactato de Sódio; $129 \mathrm{mg}$ de efedrina e uma diurese de $3,15 \mathrm{ml} / \mathrm{Kg}$ (Gráficos 6, 7 e 8 e Quadro 2).

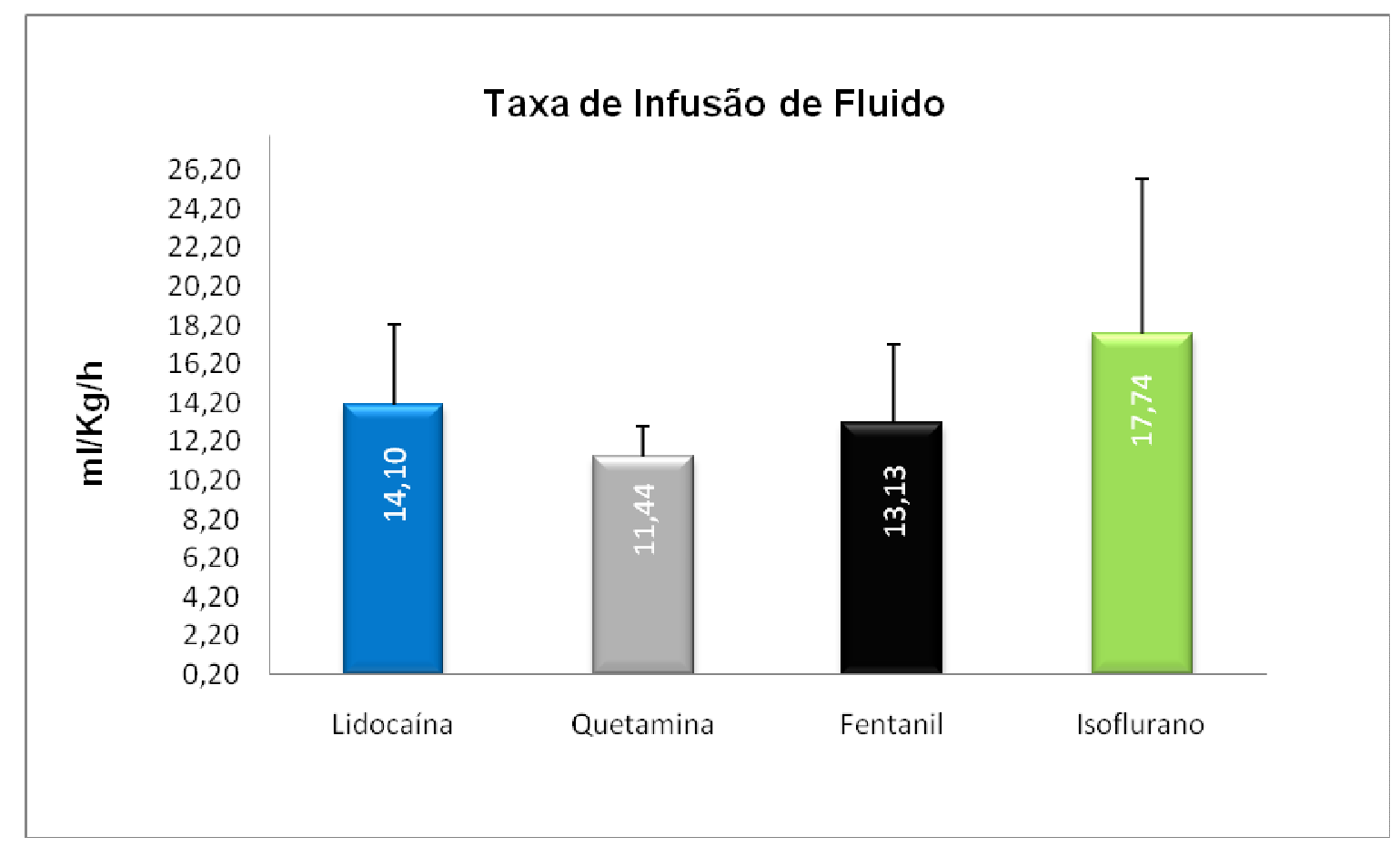

Gráfico 6 - Média e desvio padrão da taxa de infusão de fluido ( $\mathrm{m} / \mathrm{Kg} / \mathrm{h}$ ) de Ringer Lactato de Sódio em todos os animais dos grupos GL; GQ; GF e GI 


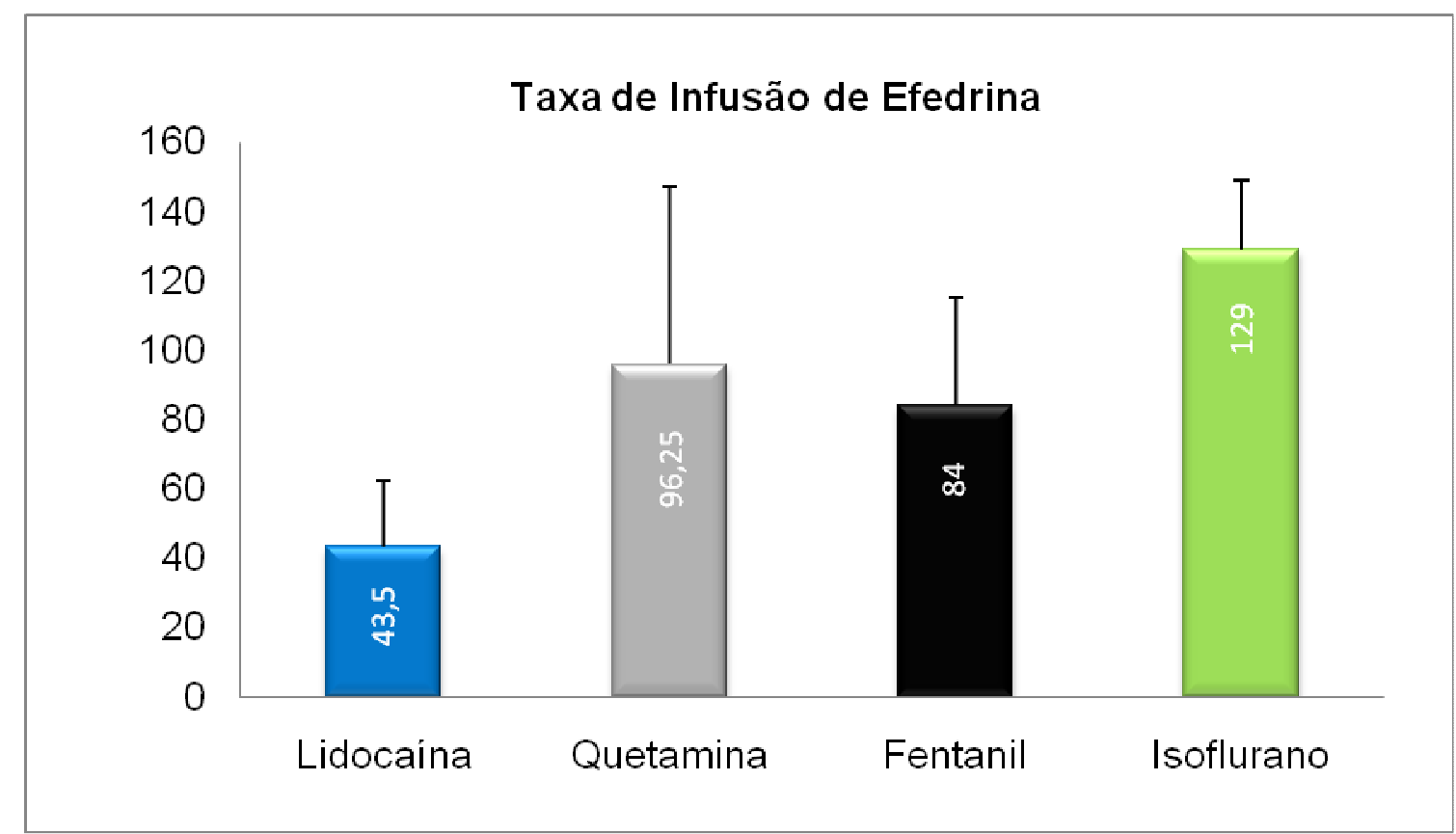

Gráfico 7 Média e desvio padrão da taxa de infusão de efedrina (mg) em todos os animais dos grupos : GL; GQ; GF e Gl

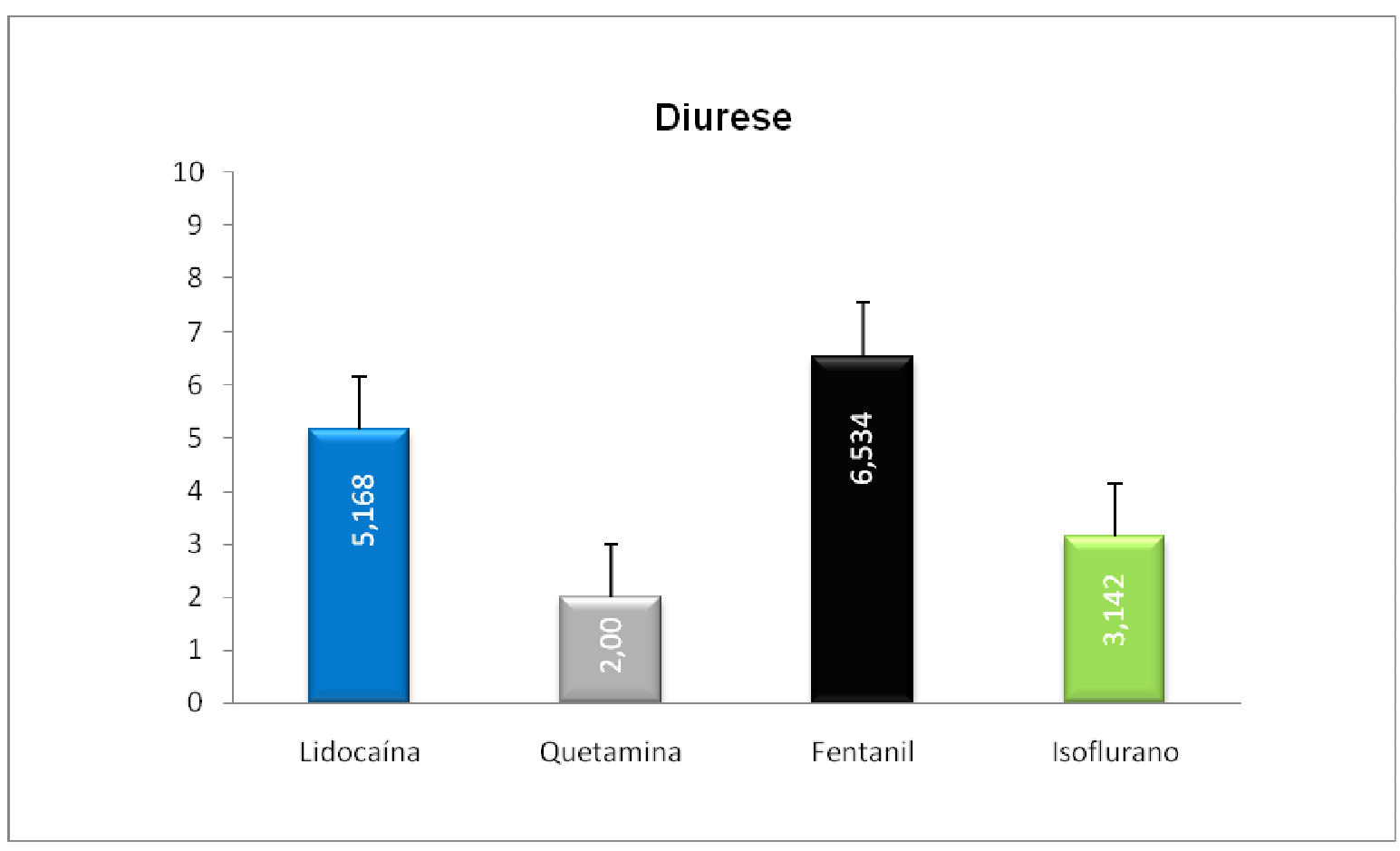

Gráfico 8 - Média e desvio padrão da diurese $(\mathrm{ml} / \mathrm{Kg} / \mathrm{h})$ em todos os animais dos grupos: GL; GQ; GF e GI 


\subsection{PARÂMETROS METABÓLICOS}

\section{Análise Entre Momentos}

\section{$\underline{\mathrm{pH}, \mathrm{HCO}_{3}}$ e $\mathrm{BE}$ do sangue arterial}

Os dados de $\mathrm{pH}, \mathrm{HCO}_{3}$ e $\mathrm{BE}$, obtidos nos grupos estudados permaneceram dentro dos níveis normais durante o experimento (Tabela 3 ).

Temperatura

Com relação a este parâmetro, observou-se no grupo isoflurano (Gl), uma queda significativa, 65 minutos após o início da anestesia inalatória, momento 30BOLUS $\left(37,4 \pm 0,28^{\circ} \mathrm{C}\right)$, quando confrontado com o momento 20INAL $(38,1 \pm 0,15)$ com $p<0,05$. Foi observada também uma queda neste parâmetro, 95 minutos após 0 início da anestesia inalatória (45BOLUS) $\left(37,1 \pm 0,50^{\circ} \mathrm{C}\right)$ em relação aos momentos 20INAL $\left(38,1 \pm 0,15^{\circ} \mathrm{C}\right)$ sendo $\mathrm{p}<0,01$ e 35 minutos após o início da anestesia inalatória APBOLUS $\left(38,0 \pm 0,28^{\circ} \mathrm{C}\right) \quad p<0,01$. A maior queda deste parâmetro foi observada após 1hora e 30 minutos após o início da anestesia inalatória, 75BOLUS $\left(36,8 \pm 0,50^{\circ} \mathrm{C}\right)$, quando confrontado com os momentos, 20INAL $\left(38,1 \pm 0,15^{\circ} \mathrm{C}\right) \mathrm{p}<$ $0,001,35$ minutos após o início da anestesia inalatória APBOLUS $\left(38,0 \pm 0,28^{\circ} \mathrm{C}\right)$ sendo $p<0,001$ e 50 minutos após o início da anestesia inalatória 15BOLUS $\left(37,7 \pm 0,19^{\circ} \mathrm{C}\right)$ com $p<0,01$ (Tabela 3 ).

\section{Análise Entre grupos}

\section{Temperatura}

Não houve diferença estatística.

\subsection{PARÂMETROS RELACIONADOS À VENTILAÇÃO}

Frequência respiratória, $\mathrm{PaCO}_{\underline{2}}, \mathrm{ETCO}_{\underline{2}}$, Pplatô, Vex, PEEP, Rva 
Em relação aos parâmetros, frequência respiratória (FR), pressão parcial de oxigênio no sangue arterial $\left(\mathrm{PaCO}_{2}\right)$, concentração de dióxido de carbono no ar expirado $\left(\mathrm{ETCO}_{2}\right)$, pressão de platô (Pplatô), volume expiratório (Vex),pressão positiva no final da expiração (PEEP) e resistência das vias aéreas (Rva), não houve diferença significativa entre momentos e grupos estudados (Tabela 4).

\section{Complacência Estática}

\section{Análise Entre Momentos}

Não houve diferença significativa entre momentos.

\section{Complacência Estática}

\section{Análise Entre grupos}

No que diz respeito ao parâmetro complacência estática (Cest), observou-se uma queda significativa no grupo fentanil (GF) em todos os momentos estudados, quando confrontado com o grupo quetamina (GQ).

No momento inicial do estudo 20INAL, este parâmetro, no grupo fentanil (GF) apresentou valor de $(329 \pm 65,18)$ e no grupo quetamina $G Q(538 \pm 75,91)$, no momento APBOLUS, estes valores foram, no grupo fentanil GF $(338 \pm 65,04)$ e no (GQ) $(603 \pm 62,38)$. Após 30 minutos da administração do bolus dos adjuvantes (15Bolus), o grupo fentanil GF apresentou o valor de Cest de $(340 \pm 61,36)$ e o grupo quetamina (GQ) $(578 \pm 62,11)$. Quando estes grupos foram confrontados no momento 30BOLUS, o valor para o grupo fentanil foi de $(347 \pm 57,99)$ e para o GQ $(590 \pm 68,76)$. Já aos 45 minutos após a administração do bolus dos adjuvantes os valores encontrados foram para o grupo fentanil GF $(348 \pm 56,18)$ e para o GQ $(582 \pm 67,87)$. Ao final do estudo, no momento 75BOLUS os valores encontrados foram: GF $(329 \pm 73,85)$ e GQ $(599 \pm 78,74)$, sendo todos os momentos o valor de $p<0,001$ (Gráfico 9 e Tabela 4). 


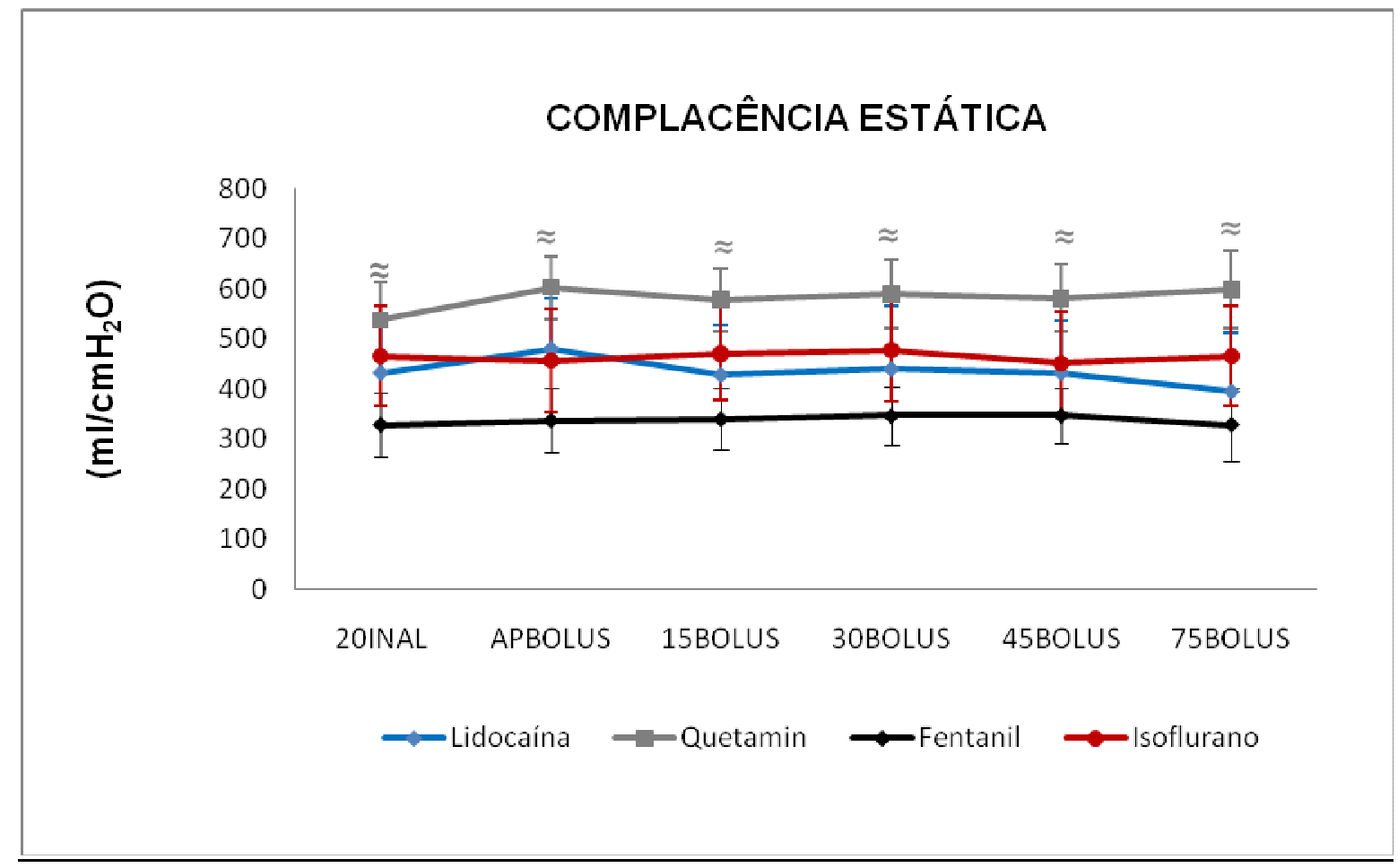

Gráfico 9 - Variação de complacência estática (Cest) (média e desvio padrão) em equinos dos grupos: Lidocaína (GL); Quetamina(GQ); Fentanil (GF) e Isoflurano (GI) ; diferiu estatisticamente do grupo fentanil (GF)

\subsection{ESCORE DE RECUPERAÇÃO}

Em relação à qualidade de recuperação, não houve diferença estatisticamente significativa entre os grupos estudados (Gráfico 10 e Quadro 2). 


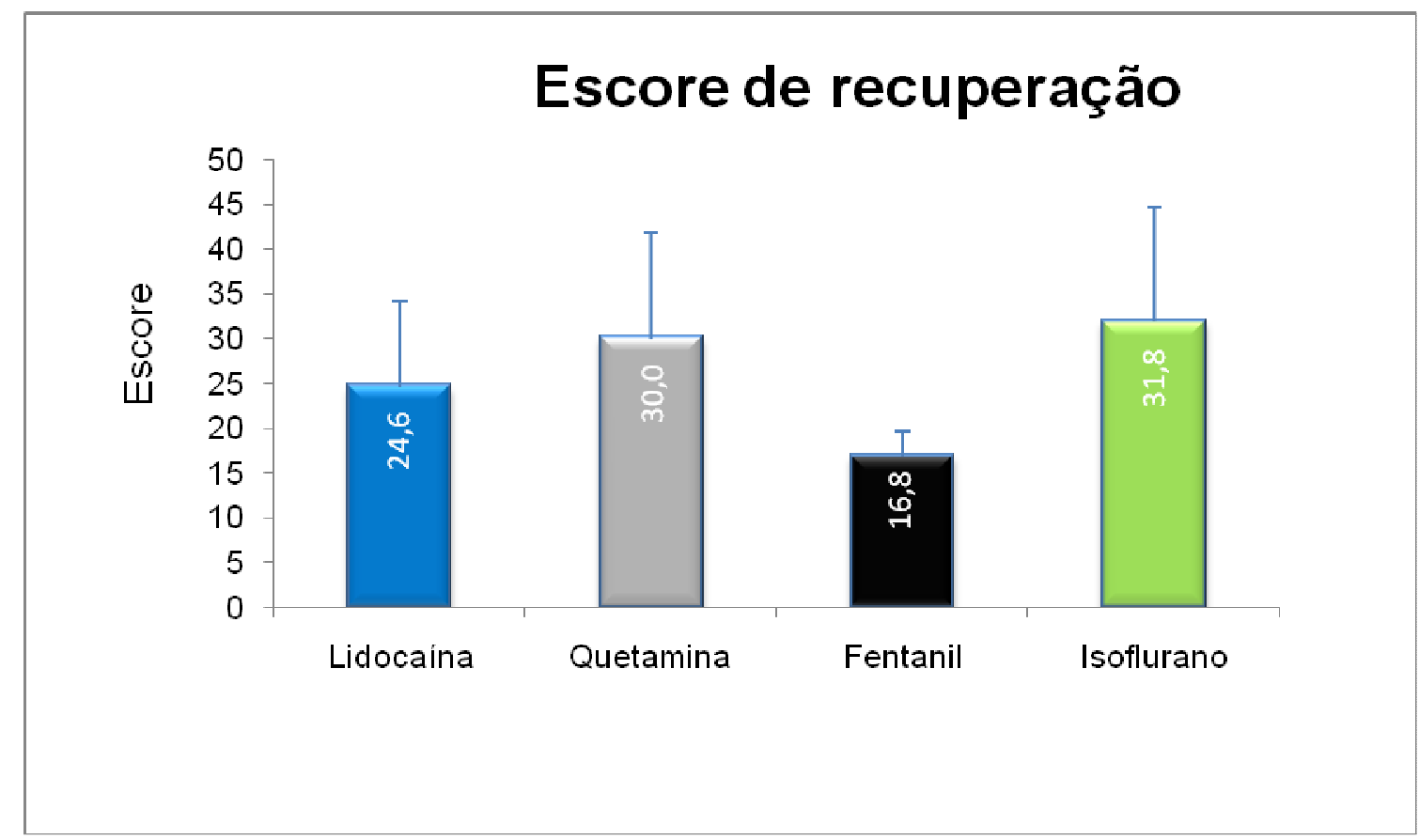

Gráfico -10 Média e desvio padrão do escore de recuperação dos animais dos grupos; GL; GQ; GF e GI 
Quadro 2- Valores referentes a peso $(\mathrm{kg})$; consumo de efedrina $(\mathrm{mg})$; taxa de infusão de fluido (ml/kg/h) e diurese $(\mathrm{ml} / \mathrm{kg} / \mathrm{h})$ dos animais nos grupos lidocaína

\begin{tabular}{|c|c|c|c|c|c|}
\hline VARIÁVEIS & ANIMAIS & $\begin{array}{c}\text { Grupo Lidocaína } \\
\text { (GL) }\end{array}$ & $\begin{array}{c}\text { Grupo Quetamina } \\
\text { (GQ) }\end{array}$ & $\begin{array}{l}\text { Grupo Fentanil } \\
\text { (GF) }\end{array}$ & $\begin{array}{c}\text { Grupo Isoflurano } \\
\text { (Gl) }\end{array}$ \\
\hline PESO & $\begin{array}{c}1 \\
2 \\
3 \\
4 \\
5 \\
\text { MÉDIA } \\
\text { DP }\end{array}$ & $\begin{array}{c}540 \\
620 \\
310 \\
330 \\
335 \\
427,0 \\
142,8\end{array}$ & $\begin{array}{c}530 \\
600 \\
567 \\
560 \\
470 \\
549,2 \\
55,6\end{array}$ & $\begin{array}{c}368 \\
368 \\
450 \\
390 \\
515 \\
418,2 \\
63,7\end{array}$ & $\begin{array}{c}470 \\
300 \\
540 \\
500 \\
580 \\
478 \\
107,8\end{array}$ \\
\hline $\begin{array}{c}\text { CONSUMO DE } \\
\text { EFEDRINA } \\
(\mathrm{mg})\end{array}$ & $\begin{array}{c}1 \\
2 \\
3 \\
4 \\
5 \\
\text { MÉDIA } \\
\text { DP }\end{array}$ & $\begin{array}{c}57,0 \\
127,5 \\
82,5 \\
59,5 \\
30,0 \\
43,5 \\
19,1\end{array}$ & $\begin{array}{c}60 \\
30 \\
135 \\
45 \\
145 \\
96,25 \\
51,1\end{array}$ & $\begin{array}{c}60 \\
90 \\
135 \\
75 \\
60 \\
84 \\
31,1\end{array}$ & $\begin{array}{l}150 \\
120 \\
120 \\
105 \\
150 \\
129 \\
20,1\end{array}$ \\
\hline $\begin{array}{c}\text { TAXA DE INFUSÃO DE } \\
\text { FLUIDO } \\
(\mathrm{ml} / \mathrm{Kg} / \mathrm{h})\end{array}$ & $\begin{array}{c}1 \\
2 \\
3 \\
4 \\
5 \\
\text { MÉDIA } \\
\text { DP }\end{array}$ & \begin{tabular}{c|}
14,8 \\
8,6 \\
15,1 \\
12,1 \\
19,9 \\
14,1 \\
4,2
\end{tabular} & $\begin{array}{c}13,80 \\
12,20 \\
10,58 \\
10,71 \\
9,92 \\
11,44 \\
1,56\end{array}$ & $\begin{array}{c}14,49 \\
16,30 \\
7,40 \\
17,09 \\
10,35 \\
13,13 \\
4,13\end{array}$ & \begin{tabular}{c|}
18,43 \\
31,11 \\
13,58 \\
10,66 \\
14,94 \\
17,74 \\
7,98
\end{tabular} \\
\hline $\begin{array}{l}\text { DIURESE } \\
(\mathrm{ml} / \mathrm{Kg} / \mathrm{h})\end{array}$ & $\begin{array}{c}1 \\
2 \\
3 \\
4 \\
5 \\
\text { MÉDIA } \\
\text { DP }\end{array}$ & \begin{tabular}{c|}
2,22 \\
4,30 \\
4,94 \\
3,44 \\
10,94 \\
5,17 \\
3,38
\end{tabular} & $\begin{array}{l}1,25 \\
1,33 \\
0,00 \\
4,46 \\
1,41 \\
2,00 \\
1,65\end{array}$ & $\begin{array}{c}14,49 \\
5,43 \\
5,92 \\
6,83 \\
0,00 \\
6,53 \\
5,19\end{array}$ & $\begin{array}{l}4,26 \\
1,33 \\
3,21 \\
2,66 \\
4,25 \\
3,14 \\
1,22 \\
\end{array}$ \\
\hline
\end{tabular}

(GL), quetamina (GQ), fentanil (GF) e isoflurano (GI). 
Tabela 1 - Parâmetros hemodinâmicos diretos dos animais dos grupos, lidocaína (GL), quetamina (GQ), fentanil (GF) e isoflurano (GI) (média \pm dp) - São Paulo - 2009

\begin{tabular}{|c|c|c|c|c|c|c|c|}
\hline VARIÁVEIS & GRUPO & 20INAL & APBOLUS & 15BOLUS & 30BOLUS & 45BOLUS & 75BOLUS \\
\hline \multirow{4}{*}{$\begin{array}{c}\text { FC } \\
\text { (bpm) }\end{array}$} & $\mathrm{GL}$ & $45,6 \pm 7,47$ & $43,2 \pm 4,92$ & $45,0 \pm 6,04$ & $44,8 \pm 5,45$ & $41,0 \pm 3,67$ & $45,2 \pm 12,15$ \\
\hline & $\mathrm{GQ}$ & $43,2 \pm 4,82$ & $41,4 \pm 8,88$ & $43,8 \pm 9,65$ & $44,2 \pm 8,01$ & $42,4 \pm 7,83$ & $43,8 \pm 7,66$ \\
\hline & GF & $45,0 \pm 6,00$ & $44,0 \pm 7,25$ & $44,4 \pm 6,66$ & $45,4 \pm 3,58$ & $45,0 \pm 4,30$ & $43,0 \pm 4,85$ \\
\hline & $\mathrm{Gl}$ & $33,8 \pm 3,03$ & $34,8 \pm 1,48$ & $39,4 \pm 5,64$ & $42,2 \pm 3,78$ & $45,0 \pm 3,54$ & $44,8 \pm 5,12$ \\
\hline \multirow{4}{*}{$\begin{array}{c}\text { PAS } \\
(\mathrm{mmHg})\end{array}$} & $\mathrm{GL}$ & $77,0 \pm 8,80$ & $86,4 \pm 13,46$ & $86,0 \pm 10,82$ & $87,4 \pm 10,78$ & $86,0 \pm 13,51$ & $81,6 \pm 11,15$ \\
\hline & $\mathrm{GQ}$ & $66,6 \pm 11,46$ & $79,0 \pm 10,12$ & $86,6 \pm 10,69$ & $90,2 \pm 15,64$ & $86,4 \pm 15,32$ & $101,0 \pm 17,26^{*}$ \\
\hline & GF & $72,0 \pm 19,60$ & $79,2 \pm 17,70$ & $89,4 \pm 14,47$ & $88,2 \pm 8,07$ & $92,0 \pm 5,43$ & $91,2 \pm 13,18$ \\
\hline & $\mathrm{Gl}$ & $51,50 \pm 9,33$ & $59,0 \pm 16,75$ & $60,5 \pm 12,77$ & $64,5 \pm 9,26$ & $70,30 \pm 8,46$ & $66,3 \pm 20,17^{*}$ \\
\hline \multirow{4}{*}{$\begin{array}{c}\text { PAM } \\
(\mathrm{mmHg})\end{array}$} & $\mathrm{GL}$ & $56,2 \pm 3,77$ & $66,8 \pm 9,68$ & $96,6 \pm 7,06$ & $71,4 \pm 6,91$ & $71,0 \pm 10,30$ & $68,0 \pm 16,09$ \\
\hline & GQ & $52,2 \pm 8,98$ & $62,0 \pm 9,95$ & $70,4 \pm 11,72$ & $71,8 \pm 12,76$ & $72,2 \pm 15,07$ & $84,4 \pm 18,93^{*}$ \\
\hline & GF & $50,8 \pm 12,11$ & $55,6 \pm 8,41$ & $67,8 \pm 5,93$ & $63,2 \pm 5,67$ & $67,0 \pm 4,06$ & $63,8 \pm 11,95$ \\
\hline & $\mathrm{Gl}$ & $52,4 \pm 4,83$ & $57,8 \pm 2,86$ & $61,2 \pm 6,83$ & $67,2 \pm 7,09$ & $67,0 \pm 8,0$ & $75,8 \pm 14,13^{*} \bullet$ \\
\hline \multirow{4}{*}{$\begin{array}{c}\text { PAD } \\
(\mathrm{mmHg})\end{array}$} & $\mathrm{GL}$ & $46,4 \pm 2,70$ & $56,0 \pm 8,15$ & $63,4 \pm 7,33$ & $60,0 \pm 7,11$ & $60,2 \pm 9,88$ & $57,6 \pm 16,88$ \\
\hline & $\mathrm{GQ}$ & $43,8 \pm 9,09$ & $51,8 \pm 9,83$ & $60,6 \pm 11,67$ & $61,4 \pm 11,28$ & $62,8 \pm 14,27$ & $73,0 \pm 19,10^{*} \approx$ \\
\hline & GF & $39,2 \pm 10,50$ & $44,0 \pm 3,81$ & $55,8 \pm 2,95^{*}$ & $49,6 \pm 4,22$ & $52,4 \pm 4,56 *$ & $49,4 \pm 9,66$ \\
\hline & $\mathrm{GI}$ & $42,6 \pm 6,35$ & $46,0 \pm 2,45$ & $48,6 \pm 5,81$ & $53,4 \pm 4,45$ & $53,6 \pm 4,98$ & $63,2 \pm 11,67^{*} \bullet € \approx$ \\
\hline
\end{tabular}

FC: freqüência cardíaca; PAS: pressão arterial sistólica; PAM: pressão arterial média; PAD: pressão arterial diastólica *Difere estatisticamente de 20inal $(p<0,05) \cdot$ Difere estatisticamente de apBolus $(p<0,05) €$ Difere estatisticamente de 15Bolus $(p<0,05) \approx D i f e r e$ estatisticamente do GF $(p<0,05)$ 
Tabela 2 - Parâmetros de oxigenação e fração expirada de isoflurano dos animais dos grupos, lidocaína (GL), quetamina (GQ), fentanil (GF) e isoflurano (Gl) (média \pm dp) - São Paulo - 2009

\begin{tabular}{|c|c|c|c|c|c|c|c|}
\hline VARIÁVEIS & GRUPO & 20INAL & APBOLUS & 15BOLUS & 30BOLUS & 45BOLUS & 75BOLUS \\
\hline $\begin{array}{c}\mathrm{PaO}_{2} \\
(\mathrm{mmHg})\end{array}$ & $\begin{array}{l}\mathrm{GL} \\
\mathrm{GQ} \\
\mathrm{GF} \\
\mathrm{Gl}\end{array}$ & $\begin{array}{c}283 \pm 119,57 \\
285 \pm 121,20 \\
300 \pm 19,53 \\
311 \pm 124,21\end{array}$ & $\begin{array}{c}318 \pm 144,11 \\
289 \pm 116,46 \\
292 \pm 58,90 \\
316 \pm 109,42\end{array}$ & $\begin{array}{c}342 \pm 144,86 \\
332 \pm 108,60 \\
263 \pm 81,90 \\
324 \pm 106,12\end{array}$ & $\begin{array}{c}348 \pm 127,42 \\
341 \pm 109,75 \\
289 \pm 74,80 \\
322 \pm 114,41\end{array}$ & $\begin{array}{c}348 \pm 134,51 \\
333 \pm 103,14 \\
296 \pm 62,54 \\
324 \pm 110,23\end{array}$ & $\begin{array}{c}312 \pm 136,11 \\
343 \pm 114,85 \\
297 \pm 70,32 \\
308 \pm 09,18\end{array}$ \\
\hline $\begin{array}{c}\mathrm{SaO}_{2} \\
(\mathrm{mmHg})\end{array}$ & $\begin{array}{l}\mathrm{GL} \\
\mathrm{GQ} \\
\mathrm{GF} \\
\mathrm{Gl}\end{array}$ & $\begin{array}{c}99,0 \pm 1,79 \\
98,6 \pm 3,13 \\
100 \pm 0,00 \\
99,0 \pm 1,79\end{array}$ & $\begin{array}{c}99,0 \pm 1,34 \\
99,6 \pm 0,89 \\
100 \pm 0,45 \\
100 \pm 0,89\end{array}$ & $\begin{array}{c}100 \pm 0,45 \\
99,8 \pm 0,45 \\
100 \pm 0,45 \\
100 \pm 0,89\end{array}$ & $\begin{array}{c}100 \pm 0,45 \\
99,8 \pm 0,45 \\
100 \pm 0,45 \\
100 \pm 0,45\end{array}$ & $\begin{array}{c}100 \pm 0,89 \\
99,8 \pm 0,45 \\
100 \pm 0,45 \\
100 \pm 0,89\end{array}$ & $\begin{array}{c}100 \pm 0,89 \\
99,8 \pm 0,45 \\
100 \pm 0,00 \\
98,0 \pm 3,03\end{array}$ \\
\hline $\begin{array}{c}{[\mathrm{ISO}]} \\
(\mathrm{Fexp} \%)\end{array}$ & $\begin{array}{l}\mathrm{GL} \\
\mathrm{GQ} \\
\mathrm{GF} \\
\mathrm{Gl}\end{array}$ & $\begin{array}{l}1,50 \pm 0,18 \\
1,44 \pm 0,11 \\
1,32 \pm 0,04 \\
1,50 \pm 0,19\end{array}$ & $\begin{array}{l}1,20 \pm 0,13 \\
1,24 \pm 0,23 \\
1,20 \pm 0,12 \\
1,40 \pm 0,22\end{array}$ & $\begin{array}{c}1,00 \pm 0,08^{*} \dagger \\
1,04 \pm 0,29^{*} \dagger \\
1,04 \pm 0,11^{*} \dagger \\
1,60 \pm 0,22\end{array}$ & $\begin{array}{c}0,90 \pm 0,05^{*} \dagger \\
0,96 \pm 0,15^{*} \dagger \\
0,96 \pm 0,05^{*} \bullet \dagger \\
1,70 \pm 0,25\end{array}$ & $\begin{array}{c}0,90 \pm 0,08^{*} \bullet \dagger \\
1,06 \pm 0,19 \dagger \\
1,00 \pm 0,00 * \bullet \dagger \\
1,60 \pm 0,26\end{array}$ & $\begin{array}{c}1,20 \pm 0,29 \dagger \\
1,10 \pm 0,19 \dagger \\
1,38 \pm 0,15 \Delta \dagger \\
1,70 \pm 0,10\end{array}$ \\
\hline
\end{tabular}

$\mathrm{PaO}_{2}$ : pressão parcial de oxigênio no sangue arterial; $\mathrm{SaO}_{2}$ : saturação de oxigênio no sangue arterial; [ISO] fração expirada de isoflurano; *Difere estatisticamente de 20inal $(p<0,05)$; Difere estatisticamente de apbolus $(p<0,05) ; \Delta$ difere estatisticamente de 15,30 e $45 b o l u s(p<0,001) ; \dagger$ Difere estatisticamente do grupo $\mathrm{Gl}(\mathrm{p}<0,05)$. 
Tabela 3 - Parâmetros metabólicos dos animais dos grupos, lidocaína (GL), quetamina (GQ), fentanil (GF) e isoflurano (GI) (média \pm dp) - São Paulo - 2009

\begin{tabular}{|c|c|c|c|c|c|c|c|}
\hline VALORES & GRUPO & 20INAL & APBOLUS & 15BOLUS & 30BOLUS & 45BOLUS & 75BOLUS \\
\hline $\mathrm{Ph}$ & $\begin{array}{l}\mathrm{GL} \\
\mathrm{GQ} \\
\mathrm{GF} \\
\mathrm{Gl}\end{array}$ & $\begin{array}{l}7,37 \pm 0,06 \\
7,34 \pm 0,04 \\
7,38 \pm 0,05 \\
7,35 \pm 0,03\end{array}$ & $\begin{array}{l}7,37 \pm 0,07 \\
7,34 \pm 0,03 \\
7,39 \pm 0,05 \\
7,30 \pm 0,05\end{array}$ & $\begin{array}{l}7,37 \pm 0,06 \\
7,35 \pm 0,03 \\
7,38 \pm 0,05 \\
7,31 \pm 0,04\end{array}$ & $\begin{array}{l}7,38 \pm 0,08 \\
7,35 \pm 0,03 \\
7,38 \pm 0,05 \\
7,31 \pm 0,04\end{array}$ & $\begin{array}{l}7,42 \pm 0,07 \\
7,35 \pm 0,03 \\
7,38 \pm 0,04 \\
7,30 \pm 0,04\end{array}$ & $\begin{array}{l}7,40 \pm 0,06 \\
7,35 \pm 0,04 \\
7,40 \pm 0,03 \\
7,30 \pm 0,04\end{array}$ \\
\hline $\begin{array}{c}\mathrm{HCO}_{3}^{-} \\
(\mathrm{mmol}-1)\end{array}$ & $\begin{array}{l}\mathrm{GL} \\
\mathrm{GQ} \\
\mathrm{GF} \\
\mathrm{Gl}\end{array}$ & $\begin{array}{l}25,0 \pm 1,52 \\
26,0 \pm 2,55 \\
25,6 \pm 1,34 \\
26,8 \pm 1,48\end{array}$ & $\begin{array}{l}26,0 \pm 2,17 \\
25,6 \pm 2,30 \\
26,6 \pm 2,41 \\
26,2 \pm 0,84\end{array}$ & $\begin{array}{l}26,0 \pm 1,82 \\
25,6 \pm 2,97 \\
24,8 \pm 3,03 \\
26,4 \pm 1,67\end{array}$ & $\begin{array}{l}26,0 \pm 4,18 \\
25,2 \pm 3,42 \\
27,2 \pm 0,84 \\
26,8 \pm 1,48\end{array}$ & $\begin{array}{l}28,0 \pm 2,40 \\
24,8 \pm 2,95 \\
26,6 \pm 1,79 \\
26,8 \pm 1,79\end{array}$ & $\begin{array}{l}27,0 \pm 2,17 \\
26,2 \pm 3,63 \\
27,2 \pm 3,11 \\
28,0 \pm 1,41\end{array}$ \\
\hline $\begin{array}{c}\mathrm{BE} \\
\left(\mathrm{mmol}^{-1}\right)\end{array}$ & $\begin{array}{l}\mathrm{GL} \\
\mathrm{GQ} \\
\mathrm{GF} \\
\mathrm{Gl}\end{array}$ & $\begin{array}{c}-0,4 \pm 1,82 \\
0,0 \pm 2,65 \\
0,8 \pm 1,48 \\
0,0 \pm 1,00\end{array}$ & $\begin{array}{c}0,4 \pm 2,30 \\
-0,4 \pm 2,61 \\
2,0 \pm 2,55 \\
0,2 \pm 0,84\end{array}$ & $\begin{array}{c}0,8 \pm 1,92 \\
-0,4 \pm 2,97 \\
0,0 \pm 2,24 \\
0,2 \pm 0,84\end{array}$ & $\begin{array}{c}0,4 \pm 3,29 \\
-0,4 \pm 3,29 \\
2,2 \pm 1,79 \\
0,2 \pm 0,84\end{array}$ & $\begin{array}{l}2,4 \pm 2,07 \\
0,0 \pm 2,45 \\
1,4 \pm 1,14 \\
0,2 \pm 1,30\end{array}$ & $\begin{array}{l}2,8 \pm 2,17 \\
0,2 \pm 3,27 \\
2,2 \pm 2,28 \\
0,2 \pm 1,30\end{array}$ \\
\hline $\begin{array}{c}\text { Temperatura } \\
\left({ }^{\circ} \mathrm{C}\right)\end{array}$ & $\begin{array}{l}\mathrm{GL} \\
\mathrm{GQ} \\
\mathrm{GF} \\
\mathrm{Gl}\end{array}$ & $\begin{array}{l}37,2 \pm 0,33 \\
38,0 \pm 0,29 \\
37,7 \pm 0,70 \\
38,1 \pm 0,15\end{array}$ & $\begin{array}{l}37,0 \pm 0,52 \\
37,9 \pm 0,44 \\
37,6 \pm 0,64 \\
38,0 \pm 0,28\end{array}$ & $\begin{array}{l}37,1 \pm 0,57 \\
37,8 \pm 0,41 \\
37,2 \pm 0,56 \\
37,7 \pm 0,19\end{array}$ & $\begin{array}{c}36,7 \pm 0,57 \\
37,7 \pm 0,50 \\
37,0 \pm 0,54 \\
37,4 \pm 0,30 *\end{array}$ & $\begin{array}{c}36,5 \pm 0,67 \\
37,5 \pm 0,51 \\
36,9 \pm 0,60 \\
37,1 \pm 0,50 *\end{array}$ & $\begin{array}{r}36,3 \pm 0,65 \\
37,6 \pm 0,49 \\
36,5 \pm 0,49 \\
36,8 \pm 0,50 * \bullet\end{array}$ \\
\hline
\end{tabular}

$\mathrm{HCO}_{3}$ :bicarbonato plasmático;BE; déficit de base; *Difere estatisticamente de 20inal $(p<0,05)$; $\cdot$ Difere estatisticamente de APbolus ( $\left.<<0,05\right)$; $€$ Difere estatisticamente de 15 bolus $(p<0,01)$ 
Tabela 4 - Parâmetros de ventilação dos animais dos grupos, lidocaína (GL), quetamina (GQ), fentanilL (GF) e isoflurano (Gl) ( média \pm dp) - São Paulo - 2009

Continua

\begin{tabular}{|c|c|c|c|c|c|c|c|}
\hline VARIAVEIS & GRUPO & 20INAL & APBOLUS & 15BOLUS & 30BOLUS & 45BOLUS & 75BOLUS \\
\hline $\begin{array}{c}\text { FR } \\
(\mathrm{mpm})\end{array}$ & $\begin{array}{l}\mathrm{GL} \\
\mathrm{GQ} \\
\mathrm{GF} \\
\mathrm{Gl}\end{array}$ & $\begin{array}{c}10,4 \pm 0,89 \\
10,4 \pm 0,89 \\
8,4 \pm 0,89 \\
10,0 \pm 0,00\end{array}$ & $\begin{array}{c}10,4 \pm 0,89 \\
9,6 \pm 0,89 \\
8,4 \pm 0,89 \\
9,6 \pm 0,89\end{array}$ & $\begin{array}{c}10,4 \pm 0,89 \\
9,2 \pm 1,10 \\
8,4 \pm 0,89 \\
9,2 \pm 1,10\end{array}$ & $\begin{array}{c}10,4 \pm 0,89 \\
9,2 \pm 1,10 \\
8,4 \pm 0,89 \\
9,2 \pm 1,10\end{array}$ & $\begin{array}{c}10,4 \pm 0,89 \\
9,2 \pm 1,10 \\
8,4 \pm 0,89 \\
9,2 \pm 1,10\end{array}$ & $\begin{array}{c}10,4 \pm 0,89 \\
9,2 \pm 1,10 \\
8,4 \pm 0,89 \\
9,4 \pm 0,89\end{array}$ \\
\hline $\begin{array}{c}\mathrm{PaCO}_{2} \\
(\mathrm{mmHg})\end{array}$ & $\begin{array}{l}\mathrm{GL} \\
\mathrm{GQ} \\
\mathrm{GF} \\
\mathrm{Gl}\end{array}$ & $\begin{array}{l}45,2 \pm 6,50 \\
49,3 \pm 0,83 \\
45,2 \pm 4,60 \\
43,8 \pm 7,79\end{array}$ & $\begin{array}{l}44,8 \pm 6,98 \\
49,3 \pm 3,27 \\
46,2 \pm 1,48 \\
48,0 \pm 1,87\end{array}$ & $\begin{array}{l}47,0 \pm 7,45 \\
47,5 \pm 5,50 \\
44,2 \pm 7,85 \\
47,8 \pm 1,48\end{array}$ & $\begin{array}{l}43,6 \pm 7,77 \\
50,0 \pm 5,74 \\
48,0 \pm 4,06 \\
47,0 \pm 3,16\end{array}$ & $\begin{array}{l}44,3 \pm 9,32 \\
48,5 \pm 1,66 \\
45,6 \pm 6,11 \\
48,4 \pm 3,21\end{array}$ & $\begin{array}{l}43,0 \pm 8,19 \\
48,5 \pm 5,68 \\
44,0 \pm 3,94 \\
50,0 \pm 4,95\end{array}$ \\
\hline $\begin{array}{c}\mathrm{ETCO}_{2} \\
(\mathrm{mmHg})\end{array}$ & $\begin{array}{l}\mathrm{GL} \\
\mathrm{GQ} \\
\mathrm{GF} \\
\mathrm{Gl}\end{array}$ & $\begin{array}{l}38,4 \pm 4,04 \\
39,2 \pm 2,77 \\
36,8 \pm 4,25 \\
34,2 \pm 4,88\end{array}$ & $\begin{array}{l}39,2 \pm 4,15 \\
40,2 \pm 2,86 \\
36,0 \pm 3,54 \\
39,6 \pm 2,30\end{array}$ & $\begin{array}{c}38,0 \pm 4,0 \\
39,0 \pm 2,55 \\
37,2 \pm 2,39 \\
39,0 \pm 3,74\end{array}$ & $\begin{array}{l}36,8 \pm 3,83 \\
39,2 \pm 2,77 \\
36,8 \pm 2,59 \\
39,6 \pm 3,44\end{array}$ & $\begin{array}{l}36,8 \pm 3,83 \\
38,8 \pm 2,77 \\
35,8 \pm 2,39 \\
40,0 \pm 2,83\end{array}$ & $\begin{array}{l}35,4 \pm 3,91 \\
40,4 \pm 4,93 \\
35,0 \pm 4,80 \\
41,2 \pm 4,92\end{array}$ \\
\hline $\begin{array}{c}\text { Pplatô } \\
\left(\mathrm{cmH}_{2} \mathrm{O}\right)\end{array}$ & $\begin{array}{l}\mathrm{GL} \\
\mathrm{GQ} \\
\mathrm{GF} \\
\mathrm{Gl}\end{array}$ & $\begin{array}{l}22,0 \pm 1,87 \\
20,6 \pm 2,30 \\
26,4 \pm 1,67 \\
20,4 \pm 1,67\end{array}$ & $\begin{array}{l}20,8 \pm 0,84 \\
20,0 \pm 1,87 \\
26,4 \pm 1,14 \\
20,8 \pm 1,64\end{array}$ & $\begin{array}{l}21,4 \pm 2,51 \\
21,2 \pm 1,30 \\
26,0 \pm 0,71 \\
21,2 \pm 1,79\end{array}$ & $\begin{array}{l}21,4 \pm 2,70 \\
20,8 \pm 1,10 \\
25,8 \pm 0,84 \\
21,0 \pm 1,87\end{array}$ & $\begin{array}{l}22,2 \pm 2,17 \\
20,8 \pm 1,64 \\
26,2 \pm 1,30 \\
21,2 \pm 2,77\end{array}$ & $\begin{array}{l}23,4 \pm 2,41 \\
20,8 \pm 2,86 \\
27,0 \pm 1,87 \\
21,2 \pm 2,17\end{array}$ \\
\hline
\end{tabular}

FR; frequência respiratória; $\mathrm{PaCO}_{2}$; pressão arterial de gás carbônico; $\mathrm{ETCO}_{2}$ : tensão de oxigênio no final da expiração;Pplat: pressão de platô. $\approx$ Difere estatisticamente do $G F(p<, 0,05)$ 
Continuação

\begin{tabular}{cccccccc}
\hline VARIÁVEIS & GRUPO & 20INAL & APBOLUS & 15BOLUS & 30BOLUS & 45BOLUS & $75 B O L U S$ \\
\hline Rva & GL & $1,2 \pm 0,64$ & $1,4 \pm 0,48$ & $1,2 \pm 0,46$ & $1,4 \pm 0,36$ & $1,3 \pm 0,64$ & $1,0 \pm 0,23$ \\
$\left(\mathrm{cmH}_{2} \mathrm{O} / \mathrm{L} / \mathrm{s}\right)$ & GQ & $0,8 \pm 0,22$ & $0,8 \pm 0,21$ & $0,7 \pm 0,26$ & $0,8 \pm 0,11$ & $0,8 \pm 0,08$ & $0,9 \pm 0,22$ \\
& GF & $0,9 \pm 0,49$ & $0,9 \pm 0,28$ & $0,8 \pm 0,33$ & $0,9 \pm 0,28$ & $0,9 \pm 0,26$ & $0,7 \pm 0,44$ \\
& GI & $0,8 \pm 0,49$ & $0,8 \pm 0,31$ & $0,9 \pm 0,33$ & $0,9 \pm 0,31$ & $1,1 \pm 0,34$ & $1,1 \pm 0,14$ \\
& & & & & & & \\
& & & & & & & \\
Ppico & & & & & & & \\
& GL & $26,40 \pm 1,85$ & $26,20 \pm 1,47$ & $26,20 \pm 1,94$ & $27,00 \pm 1,79$ & $27,00 \pm 1,41$ & $27,20 \pm 2,04$ \\
& GQ & $25,20 \pm 2,28$ & $24,60 \pm 1,82$ & $25,20 \pm 1,48$ & $25,20 \pm 1,48$ & $25,20 \pm 1,48$ & $25,60 \pm 1,95$ \\
& GF & $27,60 \pm 1,14$ & $27,60 \pm 1,14$ & $27,00 \pm 1,41$ & $27,00 \pm \pm 1,41$ & $27,40 \pm 1,34$ & $27,00 \pm 1,41$ \\
& GI & $24,00 \pm 1,26$ & $24,60 \pm 1,64$ & $25,20 \pm 0,40$ & $25,20 \pm 0,40$ & $25,80 \pm 0,75$ & $26,20 \pm 1,94$
\end{tabular}

Rva: resistência das vias aéreas;; Ppico: pressão de pico das vias aéreas. 
Conclusão

\begin{tabular}{|c|c|c|c|c|c|c|c|}
\hline VARIAVEIS & GRUPO & 20INAL & APBOLUS & 15BOLUS & 30BOLUS & 45BOLUS & 75BOLUS \\
\hline $\begin{array}{l}\text { V ex } \\
(\mathrm{L})\end{array}$ & $\begin{array}{l}\mathrm{GL} \\
\mathrm{GQ} \\
\mathrm{GF} \\
\mathrm{Gl}\end{array}$ & $\begin{array}{l}6,4 \pm 1,11 \\
7,3 \pm 1,13 \\
6,4 \pm 1,21 \\
6,1 \pm 1,02\end{array}$ & $\begin{array}{l}6,6 \pm 1,38 \\
7,3 \pm 0,86 \\
6,6 \pm 1,35 \\
6,2 \pm 0,99\end{array}$ & $\begin{array}{l}6,5 \pm 1,20 \\
8,2 \pm 1,31 \\
6,5 \pm 1,28 \\
6,6 \pm 1,14\end{array}$ & $\begin{array}{l}6,2 \pm 1,28 \\
8,2 \pm 1,36 \\
6,5 \pm 1,28 \\
6,6 \pm 1,03\end{array}$ & $\begin{array}{l}6,4 \pm 1,04 \\
8,1 \pm 1,51 \\
6,7 \pm 1,21 \\
6,3 \pm 1,21\end{array}$ & $\begin{array}{l}6,3 \pm 1,17 \\
8,2 \pm 1,28 \\
6,5 \pm 1,19 \\
6,5 \pm 1,03\end{array}$ \\
\hline PEEP & $\begin{array}{l}\mathrm{GL} \\
\mathrm{GQ} \\
\mathrm{GF} \\
\mathrm{Gl}\end{array}$ & $\begin{array}{l}7,0 \pm 0,00 \\
7,0 \pm 0,00 \\
7,0 \pm 0,00 \\
7,0 \pm 0,00\end{array}$ & $\begin{array}{l}7,0 \pm 0,00 \\
7,0 \pm 0,00 \\
7,0 \pm 0,00 \\
7,0 \pm 0,00\end{array}$ & $\begin{array}{l}7,0 \pm 0,00 \\
7,0 \pm 0,00 \\
7,0 \pm 0,00 \\
7,0 \pm 0,00\end{array}$ & $\begin{array}{l}7,0 \pm 0,00 \\
7,0 \pm 0,00 \\
7,0 \pm 0,00 \\
7,0 \pm 0,00\end{array}$ & $\begin{array}{l}7,0 \pm 0,00 \\
7,0 \pm 0,00 \\
7,0 \pm 0,00 \\
7,0 \pm 0,00\end{array}$ & $\begin{array}{l}7,0 \pm 0,00 \\
7,0 \pm 0,00 \\
7,0 \pm 0,00 \\
7,0 \pm 0,00\end{array}$ \\
\hline $\begin{array}{c}\text { Cest. } \\
\left(\mathrm{ml} / \mathrm{cmH}_{2} \mathrm{O}\right)\end{array}$ & $\begin{array}{l}\mathrm{GL} \\
\mathrm{GQ} \\
\mathrm{GF} \\
\mathrm{Gl}\end{array}$ & $\begin{array}{c}433 \pm 106,3 \\
538 \pm 75,91 \approx \\
329 \pm 65,18 \\
466 \pm 100,1\end{array}$ & $\begin{array}{c}480 \pm 100.2 \\
603 \pm 62,38 \approx \\
338 \pm 65,04 \\
457 \pm 103,7\end{array}$ & $\begin{array}{c}428 \pm 100,7 \\
578 \pm 62,11 \approx \\
340 \pm 61,36 \\
472 \pm 92,49\end{array}$ & $\begin{array}{c}440 \pm 125,6 \\
590 \pm 68,76 \approx \\
347 \pm 57,99 \\
478 \pm 103,5\end{array}$ & $\begin{array}{c}433 \pm 104,9 \\
582 \pm 67,87 \approx \\
348 \pm 56,18 \\
453 \pm 100,4\end{array}$ & $\begin{array}{c}430 \pm 117,6 \\
599 \pm 78,74 \approx \\
329 \pm 73,85 \\
466 \pm 99,32\end{array}$ \\
\hline
\end{tabular}

Vex: volume expirado; PEEP: pressão positiva no final da expiração; Cest: complacência estática; $\approx$ diferiu estatisticamente do grupo fentanil (GF) p<0,05. 


\section{DISCUSSÃO}

Os anestésicos inalatórios, além de promoverem os fatores essenciais para realização de anestesia cirúrgica, como hipnose, bloqueio dos reflexos autonômicos e relaxamento muscular, produzem efeitos indesejáveis, como depressão do sistema circulatório e respiratório, os quais são associados a complicações transoperatórias (JOHNSON, 1995; FANTONI, 1999).

No atual estudo não se observou alterações importantes da frequência cardíaca e da pressão arterial durante o decorrer do experimento por ação da anestesia inalatória, pois a administração dos adjuvantes, fentanil, quetamina e lidocaína, 20 minutos após o início da anestesia inalatória (APBolus), permitiram a diminuição da fração expirada do isoflurano de $21 \%, 28 \%$, 33\% respectivamente. De acordo com Steffey e Howland (1980), a depressão cardíaca produzida pelo anestésico isoflurano é dose dependente, como também a queda da pressão arterial. Sendo assim, uma das terapias utilizadas para garantir a perfusão tecidual abrange a diminuição da concentração inspirada de hologenados (MUIR, 2009). Corroborando com Marchioni (2003), como a perfusão tecidual é reduzida pela maioria dos agentes anestésicos gerais empregados em equinos, sendo tal redução atribuída pela maioria à vasodilatação e diminuição do débito cardíaco, os esforços devem ser direcionados na tentativa de se reduzir a concentração de anestésicos hologenados o máximo possível durante os procedimentos cirúrgicos nesta espécie.

Outro fator importante para a manutenção de adequada perfusão tecidual é a utilização de fármacos vasoativos, pois em equinos submetidos a procedimentos cirúrgicos eletivos, provavelmente a causa da hipotensão é a diminuição da resistência vascular sistêmica promovida pelos anestésicos inalatórios, mais especificamente o isoflurano (LEE et al., 2002). Neste sentido, o emprego de agentes com ação predominante em receptores $\alpha$ adrenérgicos seria uma boa opção para o controle ou a restauração da pressão arterial. Segundo (MARCHIONI, 2003) a pressão arterial média considerada satisfatória no transoperatório em equinos, deve oscilar entre 70 a $100 \mathrm{mmHg}$. Quando inferior a 70 $\mathrm{mmHg}$ caracteriza o estado de hipotensão, o qual deve ser revertido mediante 
diminuição da profundidade anestésica, fluidoterapia intravenosa e terapêutica medicamentosa através do emprego de agentes simpatomiméticos. Sendo assim, optou-se no presente estudo em realizar a infusão de efedrina quando a pressão arterial média estivesse abaixo de $60 \mathrm{mmHg}$. Esta técnica provavelmente foi responsável pela manutenção da estabilidade dos parâmetros cardiovasculares no grupo que foi mantido apenas com isoflurano, sem administração de adjuvantes capazes de reduzir a fração expirada do mesmo. Neste grupo observou-se aumento da taxa de infusão da efedrina, com maior consumo deste fármaco durante o período avaliado, quando comparado aos outros grupos estudados ( $\mathrm{Gl}=129 \mathrm{mg}, \mathrm{GF}=84 \mathrm{mg}, \mathrm{GQ}=96 \mathrm{mg}$ e $\mathrm{GL}=43 \mathrm{mg}$ ).

Sendo assim, o aumento significativo observado nas pressões arteriais sistólica, média e diastólica nos grupos isoflurano e quetamina ao final do estudo (75 Bolus) pode ser atribuído ao efeito da efedrina. No Gl as concentrações expiradas do isoflurano foram elevadas a partir do momento 15 Bolus, isto é, 35 minutos após o início da anestesia inalatória, até o final do estudo (1,6 a 1,7\%), justificando o aumento do consumo da efedrina e no $G Q$, além da ação do vasoativo, pode-se sugerir também que houve sinergismo com o efeito simpatomimético da quetamina (VALADÃO, 2010), apesar da infusão da mesma ter sido interrompida 15 minutos após o momento 45 Bolus, sem sinais de superficialização da anestesia. Sabe-se que a efedrina é um agente simpatomimético, que age em diferentes receptores adrenérgicos ( $\alpha$ e $\beta$ mais especificamente), mimetizando as catecolaminas endógenas, possibilitando que se regule a dose para melhora do rendimento cardiocirculatório (LEE et al., 2002; GWENDOLYN.; DAVID., 2007 ).

Juntamente com o aumento do consumo de efedrina, no GI, a taxa de infusão de Ringer lactato de sódio apresentou tendência a ser maior, 17,74 $\mathrm{ml} / \mathrm{kg} / \mathrm{h}$, quando comparada ao GL $(14,10 \mathrm{ml} / \mathrm{kg} / \mathrm{h})$, GF $(13,13 \mathrm{ml} / \mathrm{kg} / \mathrm{h})$ e GQ $(11,44 \mathrm{ml} / \mathrm{kg} / \mathrm{h})$, corroborando com o fato de haver ocorrido maior depressão cardiovascular promovido pela elevada concentração expirada do agente inalatório. Segundo Muir e Hubbell (2009), durante a anestesia eqüina, a infusão do fármaco vasoativo deve ser associada ao aumento da fluidoterapia, técnica utilizada rotineiramente nesta espécie, com 0 intuito de se restabelecer os 
parâmetros hemodinâmicos sem causar constrição da microcirculação e conseqüente isquemia.

No GQ, o aumento da fração expirada de isoflurano foi menor (3\%) que nos grupos GL (33\%) e GF (38\%), no momento 75 Bolus, ou seja, 15 minutos após a interrupção da infusão dos fármacos. Porém, os animais do $G Q$ não apresentavam reflexo palpebral presente, lacrimejamento ou qualquer movimentação, parâmetros utilizados como referência de superficialização do plano anestésico (MUIR, 2007), apesar de estarem ainda sob procedimento cirúrgico. Este fato ocorreu opostamente nos grupos GL e GF, onde as frações expiradas de isoflurano obtiveram maior aumento, pois os animais destes grupos apresentaram algum sinal de superficialização da anestesia. A não ocorrência da superficialização no grupo quetamina pode ter se dado devido a meia vida de duração deste fármaco ter sido mais longa quando comparada a do fentanil e da lidocaína. Langdon et al. (2006) relatam que após a infusão contínua de quetamina, a sua meia vida de eliminação, no homem, é de $79 \pm 8$ minutos, sendo que $85 \%$ do fármaco é eliminado em 2 horas. Porém ainda segundo este autor, a meia vida de eliminação em cavalos pode ser mais curta, cerca de 42 minutos. Kaka, Klavano e Hayton (1979), também estão de acordo com este dado, pois observaram em seu estudo em cavalos que os efeitos anestésicos da quetamina podem durar cerca de 48,5 minutos, quando administrada por infusão contínua. Segundo Challapalli et al. (2005) a administração de lidocaína por via venosa em humanos, raramente produz analgesia que persiste além do tempo de infusão, sendo altamente metabolizada pelo fígado e apresentando um curto tempo de meia vida. Regula e Larenza, (2007) relatam que seu efeito perdura cerca de 30 minutos após o final de sua infusão. Valverde et al. (2005), em cavalos anestesiados com isoflurano, demonstraram que a concentração plasmática de lidocaína atingida de $800 \mathrm{ng} / \mathrm{ml}$ caiu para $340 \mathrm{ng} / \mathrm{ml}, 30$ minutos após a interrupção da infusão contínua e que cavalos acordados tiveram a mesma diminuição da concentração plasmática deste fármaco, no mesmo tempo após a sua interrupção. Darien et al. (2006), relatam também, em cavalos, que a concentração plasmática aumenta rapidamente após uma dose em bolos e decresce rapidamente também após a descontinuação da infusão contínua entre 30 e 60 minutos aproximadamente, dependendo da dose utilizada e de fatores 
como fluxo sanguíneo hepático e tempo de jejum do animal. Em nosso estudo, podemos sugerir que o efeito da lidocaína começou a ser revertido após 15 minutos de sua interrupção, na dose utilizada, pois os sinais de superficialização da anestesia já se mostravam evidentes e foi necessária a elevação da fração expirada do isoflurano. Em relação ao fentanil, o tempo de reversão de seus efeitos após a interrupção de sua infusão, foi também a semelhança da lidocaína, para a dose utilizada, de aproximadamente 15 minutos, corroborando com os achados de Sano et al. (2006), que observaram em cães uma meia vida do fentanil de 20 minutos após o término de sua infusão. Já em seres humanos, a administração de uma dose intravenosa resulta em meia vida relativamente curta, de 30 a 60 minutos, devido distribuição rápida para compartimentos periféricos. Quando administrado por tempo prolongado resulta em acúmulo nestes compartimentos e em tecido adiposo e a tolerância pode se desenvolver rapidamente (BARTOLOMÉ; LOPEZ-HERCE; FREDDI, 2007). Não há relatos da meia vida do fentanil em equinos, pois os escassos trabalhos publicados com este fármaco são estudos experimentais de farmacodinâmica e farmacocinética. $O$ único estudo clinico, realizado por Ohta et al. (2010), em cavalos sob cirurgia ortopédica anestesiados com sevoflurano e com fentanil na dose de $5 \mu \mathrm{g} / \mathrm{kg}$ em bolus seguido de infusão contínua de $0,1 \mu \mathrm{g} / \mathrm{Kg} / \mathrm{min}$, ainda em fase de publicação, não foi relatado o tempo de meia vida do fármaco. Embora a farmacocinética do fentanil por via intravenosa em cavalos tenha sido descrita, a sua eficácia clínica nesta espécie não tem sido muito bem investigada, sendo que a opinião do uso clínico deste fármaco é bastante controversa entre os estudos (SANCHEZ, 2007; THOMASY, 2007).

A redução da fração expirada de anestésico inalatório com a utilização de fármacos adjuvantes como a lidocaína e a quetamina, em cavalos, já são bem conhecidas, (MUIR; SAMS, 1992; SPADAVECCHIA et al. 2002; DIZIKITI; HELLEBREKERS; DIJK, 2003; VALVERDE et al. 2005; DARIEN et al. 2005; REGULA; LARENZA, 2007; ENDERLE et al. 2008). Dizikiti, Hellebrekers e Dijk (2003) demonstraram em seu estudo, uma diminuição de $25 \%$ no requerimento do isoflurano, quando utilizaram a concentração plasmática de lidocaína de 2,14 4,23 $\mathrm{mg} \mathrm{ml}^{-1}$ em equinos. Porém Doherty e Frazier (1998), em pôneis, demonstraram uma diminuição de $20 \%$ a $70 \%$ do requerimento de halotano com o 
uso deste fármaco em concentrações de $2-7 \mu \mathrm{g} \mathrm{m}^{-1}$, ou seja, utilizando doses de bolus de $2,5 \mathrm{mg} / \mathrm{Kg}$ seguida de infusão de $50 \mu \mathrm{g} / \mathrm{Kg} / \mathrm{min}$ ou bolus de $5 \mathrm{mg} / \mathrm{Kg}$ seguido de infusão de $100 \mu \mathrm{g} / \mathrm{Kg} / \mathrm{min}$ ), durante 60 minutos. Em nosso estudo, observamos diminuição do requerimento do fármaco inalatório de $47 \%$ após bolus de $1,5 \mathrm{mg} / \mathrm{Kg}$ de lidocaína, seguido da infusão de $150 \mu \mathrm{g} / \mathrm{Kg} / \mathrm{min}$, A administração de lidocaína (dose bolus de $1,5 \mathrm{mg} / \mathrm{Kg}$ e taxa de infusão de $40 \mu \mathrm{g} / \mathrm{Kg} / \mathrm{min}$ ) associada à quetamina $(60 \mu \mathrm{g} / \mathrm{Kg} / \mathrm{min})$ por duas horas de infusão, reduziu a concentração expirada de isofluorano em equinos submetidos à procedimento cirúrgico eletivo (ASA I, II e III), cerca de 38\% (ENDERLE et al., 2008). Já, apenas a infusão contínua de quetamina, segundo Muir e Sams (1992), foi capaz de reduzir a CAM de halotano em $37 \%$ quando a concentração plasmática atingiu $10,8 \pm 2,7 \mu \mathrm{g} / \mathrm{ml}$, valor próximo ao encontrado por nós no $\mathrm{GQ}$, com diminuição da concentração expirada de isofluorano de $43 \%$.

No atual estudo, as frações expiradas de isoflurano foram diminuídas de acordo com o fármaco adjuvante utilizado e 30 minutos após o início infusão contínua de cada um.

Em relação ao fentanil, pudemos observar diminuição máxima da fração expirada do isoflurano, cerca de 44\%, 30 minutos após o início de sua infusão (30apBolus), com bolus de $7 \mu \mathrm{g} / \mathrm{kg}$ seguida da taxa de $0,7 \mu \mathrm{g} / \mathrm{kg} / \mathrm{min}$. Ohta et al. (2010) em seu estudo clínico em cavalos de corrida submetidos a cirurgia ortopédica, utilizando bolus de fentanil na dose de $5 \mu \mathrm{g} / \mathrm{kg}$, seguida de uma infusão contínua de $0,1 \mu \mathrm{g} / \mathrm{kg} / \mathrm{min}$, o que corresponde a concentração plasmática de $6,12-7,78 \mathrm{ng} / \mathrm{ml}$, afirmou que esta infusão reduziu em $13 \%$ o requerimento do agente inalatório (sevoflurano), e que a média de utilização de fármacos vasoativas para a manutenção da pressão arterial entre 60 e $80 \mathrm{mmHg}$ durante o estudo foi significantemente menor que no grupo que utilizou só o sevoflurano. Thomasy et al. $(2006,2007)$ em estudo experimental também em cavalos concluiu que o fentanil na concentração plasmática de $13 \mathrm{ng} / \mathrm{ml}$, ou seja taxa de infusão entre 0,006 a $0,1 \mu \mathrm{g} / \mathrm{kg} / \mathrm{min}$, após bolus de 0,28 a 4,69 $\mu \mathrm{g} / \mathrm{kg}(1,8$ a 16 $\mathrm{ng} / \mathrm{ml}$ ) diminui em até $18 \%$ o requerimento do anestésico inalatório isoflurano. Porém em outro estudo realizado em cavalos, Heater et al. (2009), demonstrou que a concentração plasmática de fentanil em altas doses, acima da concentração alvo de $16 \mathrm{ng} / \mathrm{ml}$, não alterou a fração expirada de isoflurano, não 
indicando a utilização rotineira do fentanil como adjuvante anestésico em cavalos adultos. Ainda segundo Sanches et al. (2007), a redução da CAM de anestésicos inalatórios em cavalos após a administração de opióides não tem sido satisfatório quando comparado com outras espécies. Em cães, $2 \mathrm{mg} / \mathrm{kg}$ de morfina reduz a CAM do isoflurano em até $50 \%$, enquanto que a mesma dose em cavalos reduz a CAM em alguns animais, mas aumenta em outros, produzindo ainda uma indesejável recuperação anestésica. O alfentanil reduz a CAM do isoflurano em gatos, mas não altera a CAM do halotano em cavalos. Portanto a resposta de opióides parece ser espécie específica e pode estar relacionada ao tipo e distribuição dos receptores opióides nas diferentes espécies. Trabalhos iniciais compararam a distribuição, a densidade e o subtipo de opióide no sistema nervoso central (SNC), mostrando importante diferença entre cães e cavalos. Sendo assim, Thomasy et al. $(2006,2007)$, relata que a redução da CAM de isoflurano de $18 \%$ no eqüino após a administração de fentanil na concentração de $13,3 \mathrm{ng} / \mathrm{ml}$ é similar em suínos ( $14 \mathrm{ng} / \mathrm{ml}$ com redução de $25 \%$ da CAM), mas é pequena quando comparada aos estudos em outras espécies, ou seja 6 a 10 $\mathrm{ng} / \mathrm{ml}$ no cão reduz $53 \%$ a CAM do isoflurano e $10 \mathrm{ng} / \mathrm{ml}$ é capaz de reduzir $82 \%$ da CAM deste mesmo anestésico em seres humanos. Da mesma maneira, o efeito da morfina no requerimento de isoflurano em suínos, cães e primatas é também qualitativo e quantitativamente semelhante ao do fentanil (primatas >cães>suínos). Sendo assim, o fentanil foi considerado ser menos efetivo em cavalos comparados com outras espécies. Por tanto, no atual estudo, a diminuição de $44 \%$ da concentração expirada de isoflurano com as doses utilizadas de fentanil, foi conseguida provavelmente por atingir concentrações plasmáticas por volta de 13 a $16 \mathrm{ng} / \mathrm{ml}$, segundo os achados de Thomasy et al. (2006, 2007) e Heater (2009). Outra diferença importante foi à realização de estudo clínico, em animais portadores de lesões articulares, com dor moderada, fato que pode modificar a característica dos receptores opióides.

A diminuição da concentração expirada de isoflurano foi semelhante com os três adjuvantes utilizados, porém em relação à qualidade de recuperação pósanestésica, observou-se que apesar de não ter havido diferença estatística no escore avaliado entre os grupos, o GF, apresentou uma menor pontuação $(16,8)$ no que diz respeito aos parâmetros avaliados. De acordo com a escala de 
avaliação de Donaldson et al. (2000), utilizada neste estudo, os animais do GF apresentaram menor tempo para se colocarem em posição quadrupedal e não apresentaram nenhum problema em relação a força e resistência, equilíbrio e coordenação bem como não apresentaram flexões de falanges quando comparados ao GQ $(30,0), \mathrm{GL}(24,6)$ e Gl $(31,8)$. De acordo com Ohta et al. 2010, em seu estudo clínico, observou que a recuperação dos cavalos que receberam a infusão de fentanil no transoperatório na taxa de $0,1 \mu \mathrm{g} / \mathrm{kg} / \mathrm{min}$, foi traquila, não precisando de assistência e de aplicação de fármacos adicionais como xilazina. A recuperação destes animais foi classificada como aceitável sem apresentarem efeitos indesejáveis. Os demais estudos com este fármaco na espécie em questão, não relatam sobre recuperação.

É importante ressaltar que todos os animais, de todos os grupos, foram resedados com xilazina na dose de $0,5 \mathrm{mg} / \mathrm{kg}$ no momento em que apresentavam os primeiros movimentos, fazendo com que o tempo de decúbito total fosse semelhante entre eles, em torno de 45 a 60 minutos. Todos os animais também foram auxiliados até ficarem em posição quadrupedal, fato que pode ter favorecido uma melhor recuperação. Apesar do bom escore de recuperação e de não se ter observado efeitos colaterais durante a recuperação no grupo fentanil , dois animais deste grupo, no período transoperatório, apresentaram fasciculações sendo necessário o uso de resgate com quetamina para que estas fasciculações cessassem.

Os animais do grupo lidocaína GL apresentaram um escore de recuperação um pouco maior que a o grupo fentanil $(24,6)$, provavelmente devido ao tempo em que se mantiveram em decúbito e/ou ao tempo que se mantiveram na posição esternal até o momento em que assumiram a posição quadrupedal, porém apresentaram um pouco de dificuldade no que diz respeito a equilíbrio e coordenação, fatores que aumentam a pontuação na tabela de recuperação. Segundo Darien et al. (2006), em seu estudo em cavalos submetidos a laparoscopia e utilizando a infusão de lidocaína no período trans-cirurgico, alguns animais apresentaram excelente período de recuperação e outros não ,sendo estas classificada com pobre. Porém, Ringer et al. (2007), em seu estudo clínico com lidocaína e medetomidina, também em cavalos, observou que a recuperação dos dois protocolos foram boas, mas apesar do tempo de decúbito do grupo que 
recebeu medetomidina ter sido mais longa, a qualidade de recuperação foi melhor que a do grupo lidocaína.

Em relação ao $G Q$ os animais apresentaram escore de recuperação de 30,0 pontos, ou seja, maior que do GL e GF devido a uma recuperação mais turbulenta, apresentando maiores números de tentativas para atingir a posição esternal e quadrupedal e mostrando em alguns momentos da avaliação, temperamento tempestuoso, porém controlado. Enderle et al. (2008), mostraram que a utilização da quetamina associada a lidocaína como adjuvantes na anestesia geral com isoflurano, em cavalos submetidos a cirurgias eletivas, apresentaram uma boa qualidade de recuperação sem efeito colateral. Porém, no estudo de Larenza et al. (2009), que avalia a recuperação de cavalos anestesiados com isoflurano e infusão contínua apenas de quetamina na dose de $1 \mathrm{~m} / \mathrm{kg} / \mathrm{h}$, demonstrou que quanto maior o tempo da infusão pior é a recuperação de acordo com a escala analógica visual (VAS) e que segundo o escore de recuperação utilizado, também observou pontuação elevada por apresentarem maiores tentativas de atingir a posição quadrupedal, não estando relacionadas ao tempo de decúbito, mesmo sedados com xilazina $0,4 \mathrm{mg} / \mathrm{kg}$, corroborando com o atual estudo.

Os animais do grupo isoflurano $\mathrm{Gl}$, apresentaram um escore maior que todos os outros grupos estudados $(31,8)$, por apresentarem, uma recuperação mais instável, com várias tentativas de mudança de decúbito (lateral - esternal) e várias tentativas para a posição quadrupedal, além disso alguns animais caíram várias vezes antes de se levantar, apoiavam-se nas paredes e no que diz respeito ao equilíbrio e a coordenação se apresentaram hesitante com marcantes flexões das falanges dos membros pélvicos.

Apesar do fentanil ter diminuído a fração expirada de isoflurano e ter apresentado uma menor pontuação na escala de avaliação de recuperação pósanestésica, será necessário um novo estudo para avaliar a ocorrência de reações no período trans-operatório, além da determinação da concentração plasmática atingida com as doses preconizadas no atual trabalho, possibilitando assim, confrontá-lo com as pesquisas de farmacocinética e farmacodinâmica em discussão atualmente. 


\section{CONCLUSÕES}

- O Fentanil foi capaz de reduzir a fração expirada de isoflurano em $44 \%$ após 30 minutos de infusão contínua nas doses preconizadas.

- Não se observou excitação ou qualquer outro efeito colateral durante o período de recuperação anestésica no grupo fentanil, após sedação com $0,5 \mathrm{mg} / \mathrm{kg}$ de xilazina I.V

- A máxima diminuição da concentração expirada de isoflurano ocorreu após 30 minutos de infusão em todos os grupos estudados.

- O grupo fentanil e lidocaína apresentaram diminuição de seus efeitos sobre a concentração expirada de isoflurano a partir de 15 minutos da interrupção da infusão destes fármacos.

- O grupo lidocaína apresentou tendência de menor consumo de efedrina, $66 \%$, em relação ao grupo isoflurano, provavelmente por manter mais estáveis os parâmetros cardiovasculares. 


\section{REFERÊNCIAS}

ABELSON, K. S.; HOGLUND, A. U. Intravenous administered lidocainein therapeutic dose increases the intraspinal release of acetylcoline in rats. Neuroscience Letters, v. 31, n. 2 p. 93-96, 2002.

AIDA, H.; STEFFEY, E. P.; PASCOE, J. R.; YARBROUGH, T. B.; TAKAHASHI, I.; HIRAGA, A.; HOBO, S.; SMITH, B. L.; STEFFEY, M. A, JONES, J. H. Use of sevoflurane for anesthetic management of horses during thoracotomy. American Journal of Veterinary Research, v. 61, n. 11, p. 1430-1437, 2000.

ARENDT, N. L.; PETERSEN, F. S.; FLESCHER, M.; BAK, P.; BJERRING, P.; ZBINDEN, A. M. The effect of N-methyl-D-aspartato antagonist (Ketamine) on single and repeated nociceptive stimuli : a placebo-controlled experimental human sudy. Anesthesia Analgesia, v. 81, n. 1, p. 63-68, 1995.

BARBIERI, M.; COLNGHI, E.; TOMMASINO, C.; ZANGRILLO, A.; GALLI, L.; TORRIG. Efficacy of the NMDA antagonist ketamine im preemptive analgesia In: WORLD CONGRESS ON PAIN, 8., 1997, Seatle. Proceedings... Seatle: IASP, 1997. v. 8, p. 343-349.

BARTOLOMÉ, S. M.; LOPÉZ-HERCE, J. C.; FREDDI, N. Analgesia and sedation in children: practical approach for the most frequent situations. Journal of Pediatric (Rio J), v.83, n. 2, p. S71-S82, 2007.

BERRINO, L.; OLIVA, P.; MASSIMO, F.; AURILIO, C.; MAIONE, S.; GRELLA, A.; ROSSI, F. Antinociceptive effect in mice of intraperitoneal N-methyl-Daspartate receptor antagonists in the formalin test. European Journal of Pain, v.7, n. 2, p. 131-137, 2003.

BLISSITT, K. J.; RAISIS, A. L.; ADAMS, V. J.; ROGERS, K. H.; HENLEY, W. E.; YOUNG, L. E. The effects of halothane and isoflurano on cardiovascular function in dorsally recumbent horses undergoing surgery. Veterinary Anesthesia

Analgesia, v.35, n. 3, p. 208-219, 2008.

BRUNSON, D. B. Use of halothane and isoflurane in the horse. Veterinary

Clinics of North American: Equine Practice, v. 6, n. 3, p. 529-541, 1990.

CHALLAPALLI, V.; TREMOUNT-LUKATS, I. W.; Mc NICOL, E. D.; LAU, J.;

CARR, D. B. Systemic administration of local anesthetic agents to relieve 
neuropathic pain. Conchrane Datadase Systematic Reviews, v. 19, n.4, dc 003345, 2005

CLARK, L.; CLUTTON, R. E.; BLISSITT, K. J.; CHASE-TOPPING, M. E. Effects of perioperative morphine administration during halothane anaesthesia in horses, Veterinary Anesthesia Analgesia, v. 32, n. 1, p. 10-15, 2005.

CLEMENTS, J. A.; NIMMO, W. S. Pharmacokinetics and analgesic effects of Ketamine in man, Births Journal of Anaesthesia, v. 53, n. 1, p. 27-30, 1981.

CODA, B.; BAUSCH, S.; HAAS, M.; CHAVKIN, C. The hypothesis that antagonism of fentanil analgesia by 2 - chloroprocaíne is mediated bydirect action on opioid receptors. Regional Anesthesia, v. 22, n. 1, p. 43-52, 1997.

COHEN, S. P.; LEBAILLE, T.; BENHAMOU, D.; LEVRON, J. C. Respiratory effects of epidural sufentanil after cesarean section. Anaesthesia and Analgesia, v. 74, n. 5, p. 677-692, 1992.

COHEN, S. P.; MAO. J. Is the analgesic effect of systemic lidocaine mediated through opioid receptors? Acta Anesthesiologica Scandinavica, v. 47, n. 7, p. 910-911, 2003.

CORRELL, G. E.; MALEKI, J.; GRACELY, E. J.; MUIR, J.J.; HARBUT, R.E. Subanesthetic ketamine infusion therapy: a retrospective analysis of a novel therapeutic approach to complex regional pain syndrome. Pain Med, v 5, n. 3, p. 263-275, 2004.

DARIEN, J. F.; MAMA, K. R.; THOMASY, S. M.; WAGNER, A. E.; ENNS, R. M. Influence of gastrointestinal tract disease on pharmacokinetics of lidocaine after intravenous infusion in anesthetized horses. America Journal of Veterinary Research, V. 67, No. 2, p. 317-322, 2006

DAVIDSON, E. M.; CARLTON, S. M. Intraplantar injection of dextrorphan, ketamine or memantine attenuates formalin-induced behaviors. Brain Research, v.785, n. 1, p. 136-142, 1998.

DE CLIVE-LOWER, S. G.; DESMOND, J.; NORTH, J. Intravenous lignocaine anaesthesia. Anaesthesia, v. 13, n. 2, p. 138-146, 1958 
DE KLAVER, M. J.; BUCKINGHAM, M. G.; RICH, G. F. Lidocaine attenuates cytokine-induced cel injury in endothelial and vascular smooth muscle cells. Anesthesia Analgesia, v. 97, n. 2, p. 465-470, 2003.

DICKENSON, A. H. Pharmacology of pain transmission and control In:

CAMPBELL J. Pain and update review. Seattle: IASP Press, 1996, p.113-121.

DOHERTY, T. J.; FRAZIER, D. L. Effect of intravenous lidocaine on halothane minimum alveolar concentration in ponies. Equine Veterinary Journal, v.30, n. 4, p. 300-303, 1998.

DUKE, T.; CAULKETT, N. A.; TATARYN, J. M. The effect of nitrous oxide on halothane, isoflurane and sevoflurane requirements in ventilated dogs undergoing ovariohysterectomy. Veterinary Anaesthesia Analgesia, v. 33, n. 6, p. $343-350$, 2006.

DUNLOP, C. I.; HODGSON, D.S.; CHAPMAN, P.L.; STEVENS, T.; WALDRON, R. D. Cardiopulmonary effects of isoflurano and halothane in spontaneously ventilating foals. Veterinary Surgery, v. 19, n. 4, p. 415, 1990.

DYSON, D. H.; MAXIE, M. G.; SCHNURR, D. Morbidity and mortality associated with anesthetic management in small animal veterinary practice in Ontario.

Journal of the American Animal Hospital Association, v. 34, n. 4, p. 325-335, 1998.

DZIKITI, T. B.; HELLEBREKERS, L. J.; DIJK, P. Effects of intravenous lidocaine on isoflurane concentration, physiological parameters, metabolic parameters and stress- related hormones in horses undergoing surgery. Journal of Veterinary Medicine, v. 50, n. 4, p. 190-195, 2003.

EBERSPÄCHER, E.; STANLEY, S. D.; REZENDE, M.; STEFFEY E.

P.Pharmacokinetcs and tolerance of transdermal fentanil administration in foals.

Veterinary Anaesthesia and Analgesia, v. 35, n. 3, p. 249-255, 2008.

ENGELKING, L. R.; BLYDEN, G. T.; LOFSTEDT, J.; GREENBLATT, D.J. Pharmacokinetics of antipyrine, acetaminophen and lidocaine in fed and fasted horses. Journal of Veterinary Pharmacology Therapeutics, v. 10, n. 1, p. 7382, 1987. 
FANTONI, D. T.; CORTOPASSI, S. R. G.; BERNARDI, M. M. Anestésicos inalatórios. In: SPINOSA, H. S.; GÓRNIAK, S. L.; BERNARDINI, M. M.

Farmacologia aplicada à medicina veterinária. 2. ed. Rio de Janeiro:

Guanabara Koogan, 1999. p. 103-113.

FANTONI, D. T.; MASTROCINQUE, S. Fisiopatologia e controle da dor aguda. In: FANTONI, D. T.; CORTOPASSI, S. R. G. Anestesia em cães e gatos. 2. ed. São Paulo: Roca, 2010. p. 555-544.

FINNERUP, N. B.; BIERING-SORENSEN, F.; JOHANNESES, I. L.; TERKELSEN, A, J.; JUHL , G, I.; KRISTENSEN, A, D.; SØREN, H.; SINDRUP.; BACH, F, W.; JENSEN, T, S. Intravenous lidocaine relieves spinal cord injury pain: a randomized controlled trail. Anesthsiology, v.102, n. 5, p. 1023-1030, 2005.

GOODMANN, R. M.; GIULMAN, A. G.; RALL, T. W.; MURAD, F. As bases farmacológicas da terapêutica. 7. ed. Rio de Janeiro: Guanabara Koogan, 1987.

GÓRNIAK, S. L. Hipnoanalgésicos. In: SPINOSA, H. S.; GÓRNIAK, S. L.; BERNARDI, M. M. Farmacologia aplicada à medicina veterinária. 3. ed. Rio de Janeiro: Guanabara Koogan, 2002. p. 158-166.

GRANDY, J. L.; STEFFEY, E. P. Anesthesia and respiratory system. In:

SCATTER, D. H. Textbook of small animal. 2. ed. Philadelphia: W.B. Saunders, 1985. p. 2621-2633.

GWENDOLYN, L. C.; DAVID, D. M. Trauma and critical patients in: TRANQUILLI, W. J.; THURMON, J. C.; GRIMM, K. A. Lumb \& Jone's vetrinary anesthesia and analgesia. $4^{\text {th }}$ ed. lowa: Blackwell Publishing, 2007. p. 969-984.

HEATHER, K.; DIMAIO, K.; STEFFEY, E. P.; MAMA, K. R.; STANLEY, S. Effects of high plasma fentanyl concentrations on minimum alveolar concentration of isoflurano in horses. America Journal of Veterinary Research, v. 70, n. 10, p. 1193-1200, 2009.

HESS, D. Detection and monitoring of hypoxemia and oxygen therapy.

Respiratory Care, v 45, n. 1, p. 650-683, 2000. 
HICKEY, P. R.; HANSEND, D.; WESSEL, D. L.; LANG, P.; JONAS, R. A.; ELIXSON, E. M. Blunting of stress responses in the pulmonary circulation of infants by fentanyl. Anesthesia Analgesia, v. 64, n. 12, p. 1137-1142, 1985.

HUBBELL, J. A. E.; MUIR, W. W. Monitoring anesthesia in: MUIR, W. W.; HUBBELL, J. A. E. Equine anesthesia: monitoring and emergency therapy. $2^{\text {nd }}$ ed. St. Louis: Saunders. 2009. p. 149-170.

JOHNSTON, G. M.; EASTMENT, J. K.; WOOD, J. L. N.; TAYLOR, P. M. The confidential enquiry into perioperative equine fatalities (CEPEF): mortality results of phases 1 and 2. Veterinary Anaesthesia Analgesia, v. 29, n. 4, p. 159-170, 2002.

KAKA, J. S.; KLAVANO, P. A.; HAYTON, W. L. Pharmacokinetics of Ketamine in horse, American Journal of Veterinary Research, v. 40, n. 7, p. $978-981$, 1979.

KAWAMATA, M.; SUGINO, S.; NARIMATSU, E. YAMAUCHI, M.; KIYA, T.; FURUSE, S.; NAMIKI, A. Effects of systemic administration of lidocaine and QX-314 on hyperexitability of spinal dorsal horn neurons after incision in the rat. Pain, v. 122, n. 1-2, p. 68-80, 2006.

KEEGAN, R. D.; GREENE, S. A. Equine anesthesia: blood pressure and monitoring. Equine Pratice, v. 16, n. 7, p. 26-33, 1994.

KOPPERT, W.; WEIGAND, M.; NEUMANN, F.; SITTL, R.; SCHUETTLER, J.; SCHMELZ, M.; HERING, W. Perioperative intravenous lidocaine has preventive effects on postoperative pain and morphine consumption aftermajor abdominal surgery. Anesthesia Analgisia, v.98, n. 4, p. 1050-1055, 2004.

KUSHIRO, T.; YAMASHITA, K.; UMAR, M. A.; MAEHARA, S.; WAKAIKI, S.; ABE, R.; SENO, T.; TSUZUKI, K.; IZUMISAWA, Y.; MUIR, W. W. Anesthetic and cardiovascular effects of balanced anesthesia using constant rate infusion of midazolam-ketaminemedetomidine with inhalation of oxygen-sevoflurane (MKMOS anesthesia) in horses. Journal of Veterinary Medical Science, v. 67, n. 4, p. 379-384, 2005.

LAMONT , L . A.; MATHEWS, K. A. Opioids, nonsteroidal anti-inflammatories, and analgesic adjuvants. In: TRANQUILLI, W. J.; THURMON, J. C.; GRIMM, K. A. Lumb \& Jone's vetrinary anesthesia and analgesia. $4^{\text {th }}$ ed. lowa: Blackwell Publishing, 2007. p. 241-271. 
LANGDON, C. F.; GORDON, W. B.; NORA, S. M.; KENNETH, E. P.; ALLEN, J. R. Pharmacokinetics and clinical effects of subanesthetic continuous rate infusion of ketamine in awake horses. American Journal of Veterinary Research, v. 67, n. 9, 2006.

LARENZA, M. P.; LANDONI, M. F.; LEVIONNOIS, O. L.; KNOBLOCH, M.; KRONEN, P. W.; THEURILLAT, R.; SCHATZMANN, U.; THORMANN W. Stereoselective pharmacokineticsof ketamine and norketamine after racemic ketamine orS-ketamine administration during isoflurane anaesthesia in Shetland ponies. British Journal of Anaesthesia, v. 98, n. 2, p. 204-212, 2007.

LARENZA, M. P.;RINGER, S. K.; KUTTER, A. P. N.; CONROT, A.; REGULA, T.; KUMMER, M.; THORMANN, W.; BETTSCHART-WOLFENSBERGER, R. Evaluation of anesthesia recovery quality after low-dose racemic or S-ketamine infusions during anesthesia with isoflurano in horses. American Journal of Veterinary Research, v. 70, n. 6, p. 710-718, 2009.

LEE, Y-H. L.; CLARKE, K. W.; ALIBHAI, H. I. K.; SONG, D. I. The effects of ephedrine on intramuscular blood flow and other cardiopulmonary parameters in halothane-anesthetized ponies. Veterinary Anaesthesia and Analgesia, v.29, n. 4, p. 171-181, 2002.

LUNA, S. P.; TAYLOR, P. M.; WHEELER, M. J . Cardiorespiratory, endocrine and metabolic change in ponies undergoing intravenous or inhalation anaesthesi. Journal of Veterynary Pharmacological Therapy, v. 19, n. 4, p. 251-258, 1996.

LUNA, S. P.; TAYLOR, P. M.; MASSONE, F. Midazolam and Ketamine induction before halothane anesthesia in ponies: Cardiorespiratory, endocrine and metabolic change, Journal of Veterinary Pharmacology and Therapeutics, v. 20, n. 2, p. 153-159, 1997.

LUNN, J. N.; MUSHIN, W. W. Mortality associated with anaesthesia.

Anaesthesia, v.37, n. 8, p. 856, 1982.

MANOHAR, M.; GOETZ, T. E. Cerebral, renal, adrenal intestinal and pancratic circulation in conscious ponies and during 1.0, 1.5 and 2.0 minimal alveolar concentration of halothane- $\mathrm{O}_{2}$ anesthesia, American Journal Veterinary Research, v. 46, n. 12, p. 2492-2498, 1985. 
MARCHIONI, G. G. Comparação dos efeitos hemodinâmicos da efedrina ou da fenilefrina em equinos anestesiados com isoflurano. 2003. $121 \mathrm{f}$.

(Mestrado em Medicina Veterinária) - Faculdade de Medicina Veterinária e Zootecnia, Universidade de São Paulo, São Paulo, 2003.

MCEWAND, A. L.; SMITH, C.; DYAR, O.; GOODMAN, D.; SMITH, L. R.; GLASS, $O$. S. Isoflurane minimum alveolar concentration reduction by fentanil.

Anesthesiology, v.78, n. 5, p. 864-869, 1993.

MENIGAUX, C.; FLETCHER, D.; DUPONT, X.; GUIGNARD, B.; GUIRIMAND, F.; CHAUVIN, M. The Benefits of Intraoperative Small-Dose Ketamine on Postoperative Pain After Anterior Cruciate Ligament Repair. Anesthesia Analgesia, v.90, n. 1, p. 129-135, 2000.

MOON, P. F.; SCALETT, J. M.; LUDDERS, J. W.; CONWAY, T. A.; LAMB, S. V. Effect of fentanil on minimum alveiolar concentration of isoflurano in swine. Anesthesiology, v. 83, n. 2, p. 535-542, 1995.

MUIJSERS, R. B.; WAGSTAFF, A. J. Transdermal fentanyl: An updated review of its pharmacological properties and therapeutic efficacy in chronic cancer pain control. Drugs, v. 61, n. 15, p. 2289-2307, 2001.

MUIR, W. W. Cardiopulmonary resuscitation in horses. In: MUIR, W. W.; HUBBELL, J. A. E. Equine anesthesia: monitoring and emergency therapy. $2^{\text {nd }}$ ed. St. Louis: Saunders. 2009. p. 418-429.

MUIR, W. W.; HUBBELL, J. A. E. Anesthetic-associated complications . In: MUIR, W. W.; HUBBELL, J. A. E. Equine anesthesia: monitoring and emergency therapy. $2^{\text {nd }}$ ed. St. Louis: Saunders. 2009. p. 397-417.

MUIR, W. W.; SAMS, R. Effects of ketamine infusion on halothane minimal alveolar concentration in horses, American Journal of Veterinary Research, $v$. 53, n. 10, p. 1802-1806, 1992.

MUIR, W. W.; SKADA, R. T.; MILNE, D. W. Evolution of xilazine and ketamine hydrochloride for anesthesia in horses, American Journal of Veterinary Research, v. 38, n. 4 p. 195- 201, 1977. 
NAGY, I.; WOOLF, C. J. Lidocaine selectivity reduces $\mathrm{C}$ fibreevoked neuronal activity in rat spinal cord in vitro by decreasing $\mathrm{N}$-metil-D-aspartate and neurokinin receptor-mediated post-synaptic depolarizations; implications for the development of novel centrally acting analgesics. Pain, v. 64, n. 1, p. 59-70, 1996.(Epub ahead of print)

OHTA, M.; WAKUNO, A.; OKADA, J.; KODAIRA, K.; NAGATA, S. I.; ITO, M.; OKU, K. Effects of Intravenous Fentanyl Administration on End-Tidal Sevoflurane Concentrations in Thoroughbred Racehorses Undergoing Orthopedic Surgery. The Journal of Veterinary Medical Science, 2010. (Epub ahead of print).Disponível em:<http://www.jstage.jst.go.jp/article/jvms/advpub/0/1003300200/ pdf>. Acesso em: 20 mar. 2010.

ORSER, B. A.; PENNEFATHER, O. S.; MAC DONALD, J. F. Multriple mechanisms of Ketamine Blockade of N-methyl-D-aspartate receptors.

Anesthesioogy, v. 86, n. 4, p. 903-917, 1997.

PALMER, C. M.; CORK, R. C.; HAYS, R.; VAN MAREN, G.; ALVES, D. The dose response relation of intrathecal fentanil for labor analgesia. Anesthesiology, v. 88, n. 22, p.335-361, 1998.

PAPICH, M. G. Priniples of analgesics drug therapy. Seminars in veterinary medicine and surgery (small animals) v. 12, n. 2, p. 80-93, 1997.

PASCOE, P. J.; STEFFEY, E. P.; BLACK. W. D.; CLAXTON, J. M.; JACOBS, J. R.; WOLINER, M. J. Evoluction of the effect of fentanil on the minimum alveolar concentration of halothane in horses, American Journal of Veterinary Research, v. 54, n. 8, p. 1327-1332, 1993.

PERSSON, J.; SCHEININ, H.; HELLSTROM, G.; BJÖRKMAN, S.; GÖTHARSON, E.; GUSTAFSSON, L. L. Ketamine antagonises alfentanil-induced hypoventilation in healthy male volunteers. Acta Anaesthesiol Scand, v. 43, n. 7 p. 744-752, 1999.

REGULA, B-W.; M.PAULA, L. Balanced anesthesia in the equine. Clinical Thechniques in Equine Practice, Balanced anesthesia in the equine. v. 6, n.2, p. 104-110, 2007.

ROBERTSON, A. S.; SANCHEZ, L. C.; MERRITT, A. M.; DOHERTY, T. J. Effect of systemic lidocaine on visceral and somatic in conscious horses. Equine veterinary Journal, v. 37, n. 2, p. 122-127, 2005. 
SANO, T.; NISHIMURA, R.; KANAZAWA, H.; IGARASHI, E.; NAGATA,Y.; MOCHIZUKI, M.; SASAKI, N. Pharmacokinetics of fentanyl after single intravenous injection and constant rate infusion in dogs. Veterinary Anaesthesia and Analgesia, v. 33, n. 4, p. 266-273, 2006.

SANCHEZ, L. C.; ROBERTSON, S. A.; MAXWELL, L. K.; ZIENTEK, K.; COLE C. Effect of Fentanyl on Visceral and Somatic Nociception in Conscious Horses. Journal of Veterinary Internal Medice, v.21, n. 5, p. 1067-1075, 2007.

SCHATZMANN, U.; GIRARD, P. Anesthesia in the horse. Tierarztl Prax, v. 12, n. 3, p. 323-328, 1984.

SERTEYN, D.; BLAIS, D.; ABOU MADI, N. A.; COPPENS, P.; MOTTART, E.; PHILIPPART, C...Circulatory and respiratory effects of ketamine in horses anesthetized with halothane. Canadian Journal of Veterinary Research, v. 51, n. 4, p. 513-516, 1987.

SHIMIZU, W.; ANTZELEVITCH, C.; SUYAMA, K.; KURITA, T.; TAGUCHI, A.; AlHARA, N.; TAKAKI, H.; SUNAGAWA, K.; KAMAKURA, S. Effect of sodiumchannel blockers on ST segment, QRS duration, and corrected QT interval in patients whit Brugada syndrome. Journal of Cardiovascar Eletrophysiology, v. 11, n. 12, p. 1320-1329, 2000.

SLINGSBY, L. S.; WATERMAN-PEARSON, E. The post-operative effects of Ketamine after ovarihysterectomy- a comparison between pre-or post- operarative administration. Reasearch in Veterinary Science, v. 69, n. 2, p. 147-152, 2000.

SOMA, L. R.; SHIELDS, D. R. Neuroleptoanalgesia produced by fentanil and droperidrol. Journal of American Veterinary Medicine Association, v. 145, n. 9, p. 897-902, 1964.

SPADAVECCHIA, C.; STUCKI, F.; MOENS, Y.; SCHATZMANN, U. Anaesthesia in horses using halothane and intravenous ketamine-guaiphenesin: a clinical study. Veterinary Anaesthesia Analgesia, v.29, n. 1, p. 20-28, 2002.

STEFFEY, E. P. Circulatory effects of inhalation anaesthesia in dogs and horses. Proc Association Veterinary Anaesthesia Great Britain Ireland, v.10, n., p. S82-S98, 1982. 
STEFFEY, E. P.; EISELE, J. H.; BOGGOT, J. D. Internations of morphine and isoflurano in horses, American Journal of Veterinary Research, v. 64 , n.2, p. 166-175, 2003.

STEFFEY, E. P.; HOWLAND, J. R. D. Cardiovascular effects of halothane in the horses. American Journal of Veterinary Research, v. 39, n. 4, p. 611-615, 1978.

STEFFEY, E. P.; HOWLAND, J. R. D. Comparison of circulatory and respiratory effects of isoflurane and halothane anesthesia inhorses. American Journal Veterinary Research, v. 41, n. 4 - 6, p. 821-82 5, 1980.

STEFFEY, E. P.; PASCOE, P. J.; WOLINER, N.J.; BERRYMAN, E. R. Effects of xylazine hydrochloride during isoflurane - induced anesthesia in horse. America Journal of Vetrinary Research, v. 61, n. 10, p. $1225-1231,2000$.

SUCENA, M.; CACHAPUZ, I.; LOMBARDIA, E.; MAGALHÃES, A.; TIAGO, GUIMARÃES, J. Plasma concentration of lidocaine during bronchoscopy. Revista Portuguesa de Pneumologi, v.10, n. 4, p. 287-296, 2004.

TAYLOR, P. M; PASCOE,P. J.; MAMA, K. R. Diagnosing and treating pain in the horse. Where are we today? Veterinary Clinic of North America: Equine Pratice, v. 18, n. 1, p. 1-19, 2002.

TEVIK, A. The role of anesthesia in surgical mortality in horses. Nordisk Veterinaermedicin, v. 35, n. 4, p. 175-179, 1983.

THOMASY, S. M.; MAMAK, R.; WHITLEY, K.; STEFFEY, E. P.; STANLEY, S. D. The influence of general anaesthesia on pharmacokinetics of fentanil and its primary metabolite in horses. Equine veterinary Journal, v. 39, n .1, p. 54 -58, 2007.

THOMASY, S. M.; PYPENDOP, B. H.; IIKIW, J. E.; STANLEY, S.D.

Pharmacokinetics of lidocaine and its active metabolite, monoethylglycinexylidide, after intravenous administration of lidocaine to awake and iso-flurane-anesthetized cats. American Journal Veterinary Research, v.66, n.7, p. 1162-1166, 2005.

THOMASY, S. M.; SLOVIS, N.; MAXWELL, L. K.; KOLLIAS-BAKER, C. Transdermal fentanyl combination with non-steroidal anti-inflammatory drugs for 
analgesia in horses. Journal of Veterinary Internal Medicine, v.18, n. 4, p. 550554, 2004.

THOMASY, S. M.; STEFFEY, E. P.; MAMMA, K. R.; SOLANO, A.; STANLEY, S. $D$. The effect of i.v fentanil administration on minimum alveolar concentration of isoflurano in horses . Brithis Journal of Anaesthesia, v. 97, n. 2, p. 323-327, 2006.

THURMON, J. C.; TRANQUILLI, W. J.; BENSON, G. J. Perioperative pain distress. In: LUMB, W. V.; JONES, E. W. Veterinary anesthesia. 3. ed. Philadelphia: Lea \& Febiger, 1996. p. 40-62.

TRIM, C. M. Monitoring during anaesthesia: technique and interpretation. Equine Veterinary Eduaction, v. 10, n. 4, p. 207-218, 1998.

VALADÃO, C. A. A. Anestésicos dissociativos. In: FANTONI, D. T.; CORTOPASSI, S. R. G. Anestesia em cães e gatos. 2. ed. São Paulo: Roca, 2010. p. 237-245.

VALVERDE, A.; DOHERTY, T. J.; HERNANDEZ, J.; DAVIES, W. Effect of lidocaine on the minimum alveolar concentration of isoflurano in dogs. Veterinary. Anaesthesia Analgesia, v. 31, n. 4, p. 264-271, 2004.

VALVERDE, A.; GUNKEL, C.; DOHERTY, T. J.; GIGUÈRE, S.; POLLAK, A. S. Effect of a constant rate infusionof lidocaine on the quality of recovery from sevoflurane or isoflurano general anaesthesia in horses. Equine Veterinary Journal, v. 37, n. 6, p. 559-564, 2005.

WAGNER, A. E.; BRODBLELT, D. I. Arterial blood pressure monitoring in anesthetized animal.Journal of American Medical Association, v. 210, n. 9, p. 1279-1285, 1997.

WAGNER, A. E.; WALTON, J. A.; HELLYER, P. W. W.; GAYNOR, J. S.; MAMA, K. R. Use of low dose of Ketamine administration by constant rate infusion as an adjunct for postoperative analgesia in dogs. Journal of the American veterinary Medical Association, v. 221, n. 1, p. 72-75, 2002.

YAMASHITA, K.; MUIR, W. W. Intravenous anesthetic and analgesic adjuncts to inhalation anesthesia. In: MUIR, W. W.; HUBBELL, J. A. E. Equine anesthesia: 
monitoring and emergency therapy. $2^{\text {nd }}$ ed. St. Louis: Saunders, 2009. p. 260276.

YASTER, M.; KOEHLER, R. C.; TRAYTMAN, R. J. Effect of fentanil on peripheral an cerebral hemodynamics in neonatal lambs. Anesthesiology, v. 66, n. 4, p. 524-530, 1987. 


\section{APÊNDICE A- TABELAS RELACIONADAS AO GRUPO LIDOCAÍNA (GL)}

Tabela 1 - Valores individuais, média e desvio-padrão do peso $(\mathrm{kg})$, débito urinário (ml) e taxa de infusão de fluido, Ringer com Lactato de Sódio (I), dos 5 animais do grupo lidocaína - São Paulo - 2009

\begin{tabular}{cccc}
\hline ANIMAIS & $\begin{array}{c}\text { PESO } \\
(\mathrm{Kg})\end{array}$ & $\begin{array}{c}\text { D.U } \\
(\mathrm{ml})\end{array}$ & $\begin{array}{c}\text { FLUIRO } \\
(\mathrm{L})\end{array}$ \\
\hline 1 & 540 & 1800 & 12 \\
2 & 620 & 4000 & 10 \\
3 & 310 & 4600 & 7 \\
4 & 330 & 1600 & 6 \\
5 & 335 & 5500 & 10 \\
\hline MÉDIA & 427,00 & 3500 & 8,75 \\
DP & 142,81 & 1729,16 & 2,75 \\
\hline
\end{tabular}

DU = DÉBITO URINÁRIO

\section{Parâmetros Hemodinâmicos Diretos}

Tabela 2 - Valores individuais, média e desvio-padrão da freqüência cardíaca (bpm) dos 5 animais do grupo lidocaína - São Paulo - 2009

\begin{tabular}{ccccccc}
\hline ANIMAIS & 20 INAL & APBOLUS & 15 BOLUS & 30 BOLUS & 45 BOLUS & 75 BOLUS \\
\hline 1 & 37 & 36 & 35 & 38 & 38 & 37 \\
2 & 51 & 49 & 49 & 46 & 39 & 39 \\
3 & 40 & 45 & 47 & 47 & 45 & 46 \\
4 & 55 & 45 & 44 & 41 & 38 & 38 \\
5 & 45 & 41 & 50 & 52 & 45 & 66 \\
\hline MÉDIA & 46 & 43 & 45 & 45 & 41 & 45 \\
DP & 7,47 & 4,92 & 6,04 & 5,45 & 3,67 & 12,15 \\
\hline
\end{tabular}

20 INAL = 20 MINUTOS APÓS ESTABILIZAÇÃO DA ANESTESIA INALATÓRIA

APBOLUS = IMEDIATAMENTE APÓS O TÉRMINO DO BOLUS

15 BOLUS $=15$ MINUTOS APÓS BOLUS

30 BOLUS $=30$ MINUTOS APÓS BOLUS

45 BOLUS $=45$ MINUTOS APÓS BOLUS

75 BOLUS $=75$ MINUTOS APÓS BOLUS 
Tabela 3 - Valores individuais, média e desvio-padrão da pressão arterial sistólica $(\mathrm{mmHg})$ dos 5 animais do grupo lidocaína - São Paulo - 2009

\begin{tabular}{ccccccc}
\hline ANIMAIS & 20 INAL & APBOLUS & 15 BOLUS & 30 BOLUS & 45 BOLUS & 75 BOLUS \\
\hline 1 & 75 & 76 & 89 & 89 & 93 & 90 \\
2 & 64 & 71 & 71 & 73 & 80 & 80 \\
3 & 83 & 98 & 101 & 102 & 106 & 67 \\
4 & 76 & 85 & 83 & 82 & 72 & 76 \\
5 & 87 & 102 & 86 & 91 & 79 & 95 \\
\hline MÉDIA & 77,00 & 86,40 & 86,00 & 87,40 & 86,00 & 81,60 \\
DP & 8,80 & 13,46 & 10,82 & 10,78 & 13,51 & 11,15 \\
\hline
\end{tabular}

20 INAL = 20 MINUTOS APÓS ESTABILIZAÇÃO DA ANESTESIA INALATÓRIA

APBOLUS = IMEDIATAMENTE APÓS O TÉRMINO DO BOLUS

15 BOLUS $=15$ MINUTOS APÓS BOLUS

30 BOLUS $=30$ MINUTOS APÓS BOLUS

45 BOLUS $=45$ MINUTOS APÓS BOLUS

75 BOLUS $=75$ MINUTOS APÓS BOLUS

Tabela 4 - Valores individuais, média de desvio padrão da pressão arterial média $(\mathrm{mmHg})$ dos 5 animais do grupo lidocaína - São Paulo - 2009

\begin{tabular}{ccccccc}
\hline ANIMAIS & 20 INAL & APBOLUS & 15 BOLUS & 30 BOLUS & 45 BOLUS & 75 BOLUS \\
\hline 1 & 57 & 57 & 63 & 80 & 84 & 91 \\
2 & 50 & 56 & 61 & 66 & 66 & 69 \\
3 & 60 & 76 & 75 & 77 & 80 & 48 \\
4 & 56 & 75 & 73 & 64 & 64 & 59 \\
5 & 58 & 70 & 76 & 70 & 61 & 73 \\
\hline MÉDIA & 56,20 & 66,80 & 69,60 & 71,40 & 71,00 & 68,00 \\
DP & 3,77 & 9,68 & 7,06 & 6,91 & 10,30 & 16,09 \\
\hline
\end{tabular}

20 INAL = 20 MINUTOS APÓS ESTABILIZAÇÃO DA ANESTESIA INALATÓRIA APBOLUS = IMEDIATAMENTE APÓS O TÉRMINO DO BOLUS

15 BOLUS $=15$ MINUTOS APÓS BOLUS

30 BOLUS $=30$ MINUTOS APÓS BOLUS

45 BOLUS $=45$ MINUTOS APÓS BOLUS

75 BOLUS $=75$ MINUTOS APÓS BOLUS 
Tabela 5 - Valores individuais, média de desvio padrão da pressão arterial diastólica (mmHg) dos 5 animais do grupo lidocaína - São Paulo - 2009

\begin{tabular}{ccccccc}
\hline ANIMAIS & 20 INAL & APBOLUS & 15 BOLUS & 30 BOLUS & 45 BOLUS & 75 BOLUS \\
\hline 1 & 48 & 47 & 72 & 71 & 75 & 82 \\
2 & 43 & 49 & 53 & 58 & 58 & 60 \\
3 & 50 & 62 & 60 & 62 & 64 & 38 \\
4 & 46 & 66 & 64 & 52 & 55 & 46 \\
5 & 45 & 56 & 68 & 57 & 49 & 62 \\
\hline MÉDIA & 46,40 & 56,00 & 63,40 & 60,00 & 60,20 & 57,60 \\
DP & 2,70 & 8,15 & 7,33 & 7,11 & 9,88 & 16,88 \\
\hline
\end{tabular}

20 INAL = 20 MINUTOS APÓS ESTABILIZAÇÃO DA ANESTESIA INALATÓRIA

APBOLUS = IMEDIATAMENTE APÓS O TÉRMINO DO BOLUS

15 BOLUS $=15$ MINUTOS APÓS BOLUS

30 BOLUS $=30$ MINUTOS APÓS BOLUS

45 BOLUS $=45$ MINUTOS APÓS BOLUS

75 BOLUS $=75$ MINUTOS APÓS BOLUS

\section{Parâmetros de Ventilação, Metabólicos e de Temperatura}

Tabela 6 - Valores individuais, média de desvio padrão da pressão parcial de $\mathrm{O}_{2}$ no sangue arterial ( $\mathrm{mmHg}$ ) dos 5 animais do grupo lidocaína - São Paulo - 2009

\begin{tabular}{ccccccc}
\hline ANIMAIS & 20 INAL & APBOLUS & 15 BOLUS & 30 BOLUS & 45 BOLUS & 75 BOLUS \\
\hline 1 & 377 & 400 & 390 & 391 & 348 & 360 \\
2 & 75 & 90 & 102 & 144 & 144 & 100 \\
3 & 336 & 337 & 338 & 329 & 344 & 265 \\
4 & 299 & 299 & 388 & 387 & 382 & 381 \\
5 & 328 & 468 & 490 & 488 & 520 & 453 \\
\hline MÉDIA & 283 & 319 & 342 & 348 & 348 & 312 \\
DP & 119,57 & 143,11 & 144,86 & 127,42 & 134,51 & 136,11 \\
\hline
\end{tabular}

20 INAL = 20 MINUTOS APÓS ESTABILIZAÇÃO DA ANESTESIA INALATÓRIA APBOLUS = IMEDIATAMENTE APÓS O TÉRMINO DO BOLUS

15 BOLUS $=15$ MINUTOS APÓS BOLUS

30 BOLUS $=30$ MINUTOS APÓS BOLUS

45 BOLUS $=45$ MINUTOS APÓS BOLUS

75 BOLUS $=75$ MINUTOS APÓS BOLUS 
Tabela 7- Valores individuais, média de desvio padrão da saturação arterial de $\mathrm{O}_{2}$

(\%) dos 5 animais do grupo lidocaína - São Paulo - 2009

\begin{tabular}{ccccccc}
\hline ANIMAIS & 20 INAL & APBOLUS & 15 BOLUS & 30 BOLUS & 45 BOLUS & 75 BOLUS \\
\hline 1 & 100 & 100 & 100 & 100 & 100 & 100 \\
2 & 96 & 97 & 99 & 99 & 98 & 98 \\
3 & 100 & 100 & 100 & 100 & 100 & 100 \\
4 & 100 & 100 & 100 & 100 & 100 & 100 \\
5 & 100 & 100 & 100 & 100 & 100 & 100 \\
\hline MÉDIA & 99 & 99 & 100 & 100 & 100 & 100 \\
DP & 1,79 & 1,34 & 0,45 & 0,45 & 0,89 & 0,89 \\
\hline
\end{tabular}

20 INAL = 20 MINUTOS APÓS ESTABILIZAÇÃO DA ANESTESIA INALATÓRIA

APBOLUS = IMEDIATAMENTE APÓS O TÉRMINO DO BOLUS

15 BOLUS = 15 MINUTOS APÓS BOLUS

30 BOLUS $=30$ MINUTOS APÓS BOLUS

45 BOLUS $=45$ MINUTOS APÓS BOLUS

75 BOLUS $=75$ MINUTOS APÓS BOLUS

Tabela 8 - Valores individuais, média de desvio padrão da pressão parcial de $\mathrm{CO}_{2}$ no sangue arterial ( $\mathrm{mmHg}$ ) dos 5 animais do grupo lidocaína - São Paulo - 2009

\begin{tabular}{ccccccc}
\hline ANIMAIS & 20 INAL & APBOLUS & 15 BOLUS & 30 BOLUS & 45 BOLUS & 75 BOLUS \\
\hline 1 & 55 & 55 & 57 & 55 & 57 & 56 \\
2 & 46 & 47 & 47 & 39 & 38 & 38 \\
3 & 45 & 42 & 42 & 40 & 40 & 36 \\
4 & 43 & 44 & 51 & 48 & 52 & 46 \\
5 & 37 & 36 & 38 & 36 & 36 & 39 \\
\hline MÉDIA & 45 & 45 & 47 & 44 & 45 & 43 \\
DP & 6,50 & 6,98 & 7,45 & 7,77 & 9,32 & 8,19 \\
\hline
\end{tabular}

20 INAL = 20 MINUTOS APÓS ESTABILIZAÇÃO DA ANESTESIA INALATÓRIA

APBOLUS $=$ IMEDIATAMENTE APÓS O TÉRMINO DO BOLUS

15 BOLUS $=15$ MINUTOS APÓS BOLUS

30 BOLUS $=30$ MINUTOS APÓS BOLUS

45 BOLUS $=45$ MINUTOS APÓS BOLUS

75 BOLUS = 75 MINUTOS APÓS BOLUS 
Tabela 9 - Valores individuais, média de desvio padrão da freqüência respiratória (mpm) dos 5 animais do grupo lidocaína - São Paulo - 2009

\begin{tabular}{ccccccc}
\hline ANIMAIS & 20 INAL & APBOLUS & 15 BOLUS & 30 BOLUS & 45 BOLUS & 75 BOLUS \\
\hline 1 & 8 & 8 & 8 & 8 & 8 & 8 \\
2 & 10 & 10 & 10 & 10 & 10 & 10 \\
3 & 12 & 12 & 12 & 12 & 12 & 12 \\
4 & 12 & 12 & 12 & 12 & 12 & 12 \\
5 & 12 & 12 & 12 & 12 & 12 & 12 \\
\hline MÉDIA & 10,80 & 10,80 & 10,80 & 10,80 & 10,80 & 10,80 \\
DP & 1,79 & 1,79 & 1,79 & 1,79 & 1,79 & 1,79 \\
\hline
\end{tabular}

20 INAL = 20 MINUTOS APÓS ESTABILIZAÇÃO DA ANESTESIA INALATÓRIA

APBOLUS $=$ IMEDIATAMENTE APÓS O TÉRMINO DO BOLUS

15 BOLUS $=15$ MINUTOS APÓS BOLUS

30 BOLUS $=30$ MINUTOS APÓS BOLUS

45 BOLUS $=45$ MINUTOS APÓS BOLUS

75 BOLUS $=75$ MINUTOS APÓS BOLUS

Tabela 10 - Valores individuais, média de desvio padrão da concentração do anestésico inalatório no ar expirado (V\%) dos 5 animais do grupo lidocaína - São Paulo - 2009

\begin{tabular}{ccccccc}
\hline ANIMAIS & 20 INAL & APBOLUS & 15 BOLUS & 30 BOLUS & 45 BOLUS & 75 BOLUS \\
\hline 1 & 1,7 & 1,3 & 1,1 & 1,0 & 1,0 & 1,0 \\
2 & 1,6 & 1,3 & 1,0 & 1,0 & 1,0 & 1,0 \\
3 & 1,3 & 1,3 & 1,1 & 0,9 & 0,9 & 1,3 \\
4 & 1,4 & 1,0 & 0,9 & 0,9 & 0,8 & 0,9 \\
5 & 1,3 & 1,3 & 1,0 & 0,9 & 0,9 & 1,6 \\
\hline MÉDIA & 1,5 & 1,2 & 1,0 & 0,9 & 0,9 & 1,2 \\
DP & 0,18 & 0,13 & 0,08 & 0,05 & 0,08 & 0,29 \\
\hline
\end{tabular}

20 INAL = 20 MINUTOS APÓS ESTABILIZAÇÃO DA ANESTESIA INALATÓRIA

APBOLUS $=$ IMEDIATAMENTE APÓS O TÉRMINO DO BOLUS

15 BOLUS $=15$ MINUTOS APÓS BOLUS

30 BOLUS $=30$ MINUTOS APÓS BOLUS

45 BOLUS $=45$ MINUTOS APÓS BOLUS

75 BOLUS $=75$ MINUTOS APÓS BOLUS 
Tabela 11 - Valores individuais, média de desvio padrão do volume inspiratório (mmHg) dos 5 animais do grupo lidocaína - São Paulo - 2009

\begin{tabular}{ccccccc}
\hline ANIMAIS & 20 INAL & APBOLUS & 15 BOLUS & 30 BOLUS & 45 BOLUS & 75 BOLUS \\
\hline 1 & 6,5 & 6,5 & 6,5 & 6,5 & 6,5 & 6,5 \\
2 & 7,0 & 7,0 & 7,0 & 7,0 & 7,0 & 7,0 \\
3 & 4,5 & 4,5 & 4,5 & 4,5 & 4,5 & 4,5 \\
4 & 4,5 & 4,5 & 4,5 & 4,5 & 4,5 & 4,5 \\
5 & 5,5 & 5,5 & 5,5 & 5,5 & 5,5 & 5,5 \\
\hline MÉDIA & 5,6 & 5,6 & 5,6 & 5,6 & 5,6 & 5,6 \\
DP & 1,14 & 1,14 & 1,14 & 1,14 & 1,14 & 1,14 \\
\hline
\end{tabular}

20 INAL = 20 MINUTOS APÓS ESTABILIZAÇÃO DA ANESTESIA INALATÓRIA

APBOLUS = IMEDIATAMENTE APÓS O TÉRMINO DO BOLUS

15 BOLUS $=15$ MINUTOS APÓS BOLUS

30 BOLUS $=30$ MINUTOS APÓS BOLUS

45 BOLUS $=45$ MINUTOS APÓS BOLUS

75 BOLUS $=75$ MINUTOS APÓS BOLUS

Tabela 12 - Valores individuais, média de desvio padrão do volume expiratório (mmHg) dos 5 animais do grupo lidocaína - São Paulo - 2009

\begin{tabular}{ccccccc}
\hline ANIMAIS & 20 INAL & APBOLUS & 15 BOLUS & 30 BOLUS & 45 BOLUS & 75 BOLUS \\
\hline 1 & 7,57 & 7,45 & 7,59 & 7,73 & 7,73 & 7,75 \\
2 & 7,48 & 7,48 & 7,44 & 7,35 & 7,30 & 7,35 \\
3 & 5,05 & 5,07 & 5,07 & 5,23 & 5,23 & 5,27 \\
4 & 5,66 & 5,18 & 5,06 & 4,82 & 5,91 & 5,80 \\
5 & 6,14 & 7,93 & 6,06 & 6,10 & 6,02 & 5,30 \\
\hline MÉDIA & 6 & 7 & 7 & 6 & 6 & 6 \\
DP & 1,11 & 1,38 & 1,20 & 1,28 & 1,04 & 1,17 \\
\hline
\end{tabular}

20 INAL = 20 MINUTOS APÓS ESTABILIZAÇÃO DA ANESTESIA INALATÓRIA

APBOLUS = IMEDIATAMENTE APÓS O TÉRMINO DO BOLUS

15 BOLUS $=15$ MINUTOS APÓS BOLUS

30 BOLUS $=30$ MINUTOS APÓS BOLUS

45 BOLUS $=45$ MINUTOS APÓS BOLUS

75 BOLUS $=75$ MINUTOS APÓS BOLUS 
Tabela 13 - Valores individuais, média de desvio padrão do platô $\left(\mathrm{cmH}_{2} \mathrm{O}\right)$ dos 5 animais do grupo lidocaína - São Paulo - 2009

\begin{tabular}{ccccccc}
\hline ANIMAIS & 20 INAL & APBOLUS & 15 BOLUS & 30 BOLUS & 45 BOLUS & 75 BOLUS \\
\hline 1 & 20 & 21 & 21 & 20 & 21 & 22 \\
2 & 22 & 22 & 22 & 21 & 21 & 21 \\
3 & 22 & 20 & 25 & 26 & 22 & 26 \\
4 & 21 & 21 & 18 & 19 & 26 & 26 \\
5 & 25 & 20 & 21 & 21 & 21 & 22 \\
\hline MÉDIA & 22 & 21 & 21 & 21 & 22 & 23 \\
DP & 1,87 & 0,84 & 2,51 & 2,70 & 2,17 & 2,41 \\
\hline
\end{tabular}

20 INAL = 20 MINUTOS APÓS ESTABILIZAÇÃO DA ANESTESIA INALATÓRIA

APBOLUS = IMEDIATAMENTE APÓS O TÉRMINO DO BOLUS

15 BOLUS $=15$ MINUTOS APÓS BOLUS

30 BOLUS $=30$ MINUTOS APÓS BOLUS

45 BOLUS $=45$ MINUTOS APÓS BOLUS

75 BOLUS $=75$ MINUTOS APÓS BOLUS

Tabela 14 - Valores individuais, média de desvio padrão da resistência em vias aéreas $\left(\mathrm{cmH}_{2} \mathrm{O} / / / \mathrm{seg}\right.$.)) dos 5 animais do grupo lidocaína - São Paulo - 2009

\begin{tabular}{ccccccc}
\hline ANIMAIS & 20 INAL & APBOLUS & 15 BOLUS & 30 BOLUS & 45 BOLUS & 75 BOLUS \\
\hline 1 & 9 & 10 & 10 & 9 & 9 & 10 \\
2 & 8 & 8 & 8 & 8 & 8 & 7 \\
3 & 12 & 11 & 12 & 12 & 12 & 11 \\
4 & 10 & 9 & 9 & 9 & 9 & 10 \\
5 & 9 & 8 & 9 & 9 & 9 & 8 \\
\hline MÉDIA & 9,6 & 9,2 & 9,6 & 9,4 & 9,4 & 9,2 \\
DP & 1,36 & 1,17 & 1,36 & 1,36 & 1,36 & 1,47 \\
\hline
\end{tabular}

20 INAL = 20 MINUTOS APÓS ESTABILIZAÇÃO DA ANESTESIA INALATÓRIA APBOLUS $=$ IMEDIATAMENTE APÓS O TÉRMINO DO BOLUS

15 BOLUS $=15$ MINUTOS APÓS BOLUS 30 BOLUS $=30$ MINUTOS APÓS BOLUS 45 BOLUS $=45$ MINUTOS APÓS BOLUS 75 BOLUS = 75 MINUTOS APÓS BOLUS 
Tabela 15 - Valores individuais, média de desvio padrão da complacência estática $\left(\mathrm{ml} / \mathrm{cmH}_{2} \mathrm{O}\right)$ dos 5 animais do grupo lidocaína - São Paulo - 2009

\begin{tabular}{ccccccc}
\hline ANIMAIS & 20 INAL & APBOLUS & 15 BOLUS & 30 BOLUS & 45 BOLUS & 75 BOLUS \\
\hline 1 & 215 & 232 & 242 & 249 & 259 & 234 \\
2 & 318 & 316 & 296 & 301 & 310 & 315 \\
3 & 128 & 130 & 121 & 118 & 120 & 127 \\
4 & 165 & 159 & 188 & 174 & 169 & 165 \\
5 & 218 & 215 & 210 & 190 & 188 & 200 \\
\hline MÉDIA & 209 & 210 & 211 & 206 & 209 & 208 \\
DP & 71,58 & 72,02 & 64,84 & 70,53 & 75,27 & 71,76 \\
\hline
\end{tabular}

20 INAL = 20 MINUTOS APÓS ESTABILIZAÇÃO DA ANESTESIA INALATÓRIA APBOLUS $=$ IMEDIATAMENTE APÓS O TÉRMINO DO BOLUS 15 BOLUS $=15$ MINUTOS APÓS BOLUS 30 BOLUS $=30$ MINUTOS APÓS BOLUS 45 BOLUS $=45$ MINUTOS APÓS BOLUS 75 BOLUS $=75$ MINUTOS APÓS BOLUS

Tabela 16 - Valores individuais, média de desvio padrão da pressão positiva do no final da expiração $\left(\mathrm{cmH}_{2} \mathrm{O}\right)$ dos 5 animais do grupo lidocaína - São Paulo 2009

\begin{tabular}{ccccccc}
\hline ANIMAIS & 20 INAL & APBOLUS & 15 BOLUS & 30 BOLUS & 45 BOLUS & 75 BOLUS \\
\hline 1 & 7 & 7 & 7 & 7 & 7 & 7 \\
2 & 7 & 7 & 7 & 7 & 7 & 7 \\
3 & 7 & 7 & 7 & 7 & 7 & 7 \\
4 & 7 & 7 & 7 & 7 & 7 & 7 \\
5 & 7 & 7 & 7 & 7 & 7 & 7 \\
\hline MÉDIA & 7 & 7 & 7 & 7 & 7 & 7 \\
DP & 0,00 & 0,00 & 0,00 & 0,00 & 0,00 & 0,00 \\
\hline
\end{tabular}

20 INAL = 20 MINUTOS APÓS ESTABILIZAÇÃO DA ANESTESIA INALATÓRIA APBOLUS = IMEDIATAMENTE APÓS O TÉRMINO DO BOLUS

15 BOLUS $=15$ MINUTOS APÓS BOLUS

30 BOLUS $=30$ MINUTOS APÓS BOLUS 45 BOLUS $=45$ MINUTOS APÓS BOLUS 75 BOLUS $=75$ MINUTOS APÓS BOLUS 
Tabela 17 - Valores individuais, média de desvio padrão da pressão de pico inspiratória $\left(\mathrm{cmH}_{2} \mathrm{O}\right)$ dos 5 animais do grupo lidocaína - São Paulo - 2009

\begin{tabular}{ccccccc}
\hline ANIMAIS & 20 INAL & APBOLUS & 15 BOLUS & 30 BOLUS & 45 BOLUS & 75 BOLUS \\
\hline 1 & 24 & 25 & 24 & 25 & 25 & 25 \\
2 & 28 & 28 & 29 & 28 & 28 & 27 \\
3 & 29 & 28 & 28 & 30 & 29 & 30 \\
4 & 26 & 25 & 25 & 26 & 26 & 29 \\
5 & 25 & 25 & 25 & 26 & 27 & 25 \\
\hline MÉDIA & 26,4 & 26,2 & 26,2 & 27 & 27 & 27,2 \\
DP & 1,85 & 1,47 & 1,94 & 1,79 & 1,41 & 2,04 \\
\hline
\end{tabular}

20 INAL = 20 MINUTOS APÓS ESTABILIZAÇÃO DA ANESTESIA INALATÓRIA

APBOLUS $=$ IMEDIATAMENTE APÓS O TÉRMINO DO BOLUS

15 BOLUS $=15$ MINUTOS APÓS BOLUS

30 BOLUS $=30$ MINUTOS APÓS BOLUS

45 BOLUS $=45$ MINUTOS APÓS BOLUS

75 BOLUS $=75$ MINUTOS APÓS BOLUS

Tabela 18 - Valores individuais, média de desvio padrão do $\mathrm{CO}_{2}$ no final da expiração das vias aéreas $(\mathrm{mmHg})$ dos 5 animais do grupo lidocaína - São Paulo 2009

\begin{tabular}{ccccccc}
\hline ANIMAIS & 20 INAL & APBOLUS & 15 BOLUS & 30 BOLUS & 45 BOLUS & 75 BOLUS \\
\hline 1 & 44 & 45 & 44 & 42 & 42 & 41 \\
2 & 36 & 37 & 36 & 36 & 36 & 36 \\
3 & 34 & 35 & 34 & 32 & 32 & 30 \\
4 & 41 & 42 & 40 & 39 & 39 & 35 \\
5 & 37 & 37 & 36 & 35 & 35 & 35 \\
\hline MÉDIA & 38 & 39 & 38 & 37 & 37 & 35 \\
DP & 4,04 & 4,15 & 4,00 & 3,83 & 3,83 & 3,91 \\
\hline
\end{tabular}

20 INAL = 20 MINUTOS APÓS ESTABILIZAÇÃO DA ANESTESIA INALATÓRIA

APBOLUS = IMEDIATAMENTE APÓS O TÉRMINO DO BOLUS

15 BOLUS = 15 MINUTOS APÓS BOLUS

30 BOLUS $=30$ MINUTOS APÓS BOLUS

45 BOLUS = 45 MINUTOS APÓS BOLUS

75 BOLUS = 75 MINUTOS APÓS BOLUS 
Tabela 19 - Valores individuais, média de desvio padrão do $\mathrm{pH}$ do sangue arterial dos 5 animais do grupo lidocaína- São Paulo - 2009

\begin{tabular}{ccccccc}
\hline ANIMAIS & 20 INAL & APBOLUS & 15 BOLUS & 30 BOLUS & 45 BOLUS & 75 BOLUS \\
\hline 1 & 7,31 & 7,32 & 7,31 & 7,32 & 7,32 & 7,33 \\
2 & 7,31 & 7,3 & 7,33 & 7,33 & 7,42 & 7,42 \\
3 & 7,37 & 7,38 & 7,4 & 7,41 & 7,45 & 7,45 \\
4 & 7,39 & 7,38 & 7,35 & 7,38 & 7.39 & 7,43 \\
5 & 7,45 & 7,47 & 7,46 & 7,48 & 7,48 & 7,48 \\
\hline MÉDIA & 7,37 & 7,37 & 7,37 & 7,38 & 7,42 & 7,42 \\
DP & 0,06 & 0,07 & 0,06 & 0,07 & 0,07 & 0,06 \\
\hline
\end{tabular}

20 INAL = 20 MINUTOS APÓS ESTABILIZAÇÃO DA ANESTESIA INALATÓRIA

APBOLUS $=$ IMEDIATAMENTE APÓS O TÉRMINO DO BOLUS

15 BOLUS $=15$ MINUTOS APÓS BOLUS

30 BOLUS $=30$ MINUTOS APÓS BOLUS

45 BOLUS $=45$ MINUTOS APÓS BOLUS

75 BOLUS $=75$ MINUTOS APÓS BOLUS

Tabela 20 - Valores individuais, média de desvio padrão do bicarbonato no sangue arterial (mmol/l) dos 5 animais do grupo lidocaína - São Paulo - 2009

\begin{tabular}{ccccccc}
\hline ANIMAIS & 20 INAL & APBOLUS & 15 BOLUS & 30 BOLUS & 45 BOLUS & 75 BOLUS \\
\hline 1 & 27 & 27 & 28 & 27 & 28 & 28 \\
2 & 23 & 23 & 24 & 20 & 24 & 24 \\
3 & 25 & 24 & 25 & 24 & 26 & 25 \\
4 & 26 & 28 & 28 & 31 & 31 & 28 \\
5 & 26 & 27 & 27 & 28 & 29 & 29 \\
\hline MÉDIA & 25 & 26 & 26 & 26 & 28 & 27 \\
DP & 1,52 & 2,17 & 1,82 & 4,18 & 2,70 & 2,17 \\
\hline
\end{tabular}

20 INAL = 20 MINUTOS APÓS ESTABILIZAÇÃO DA ANESTESIA INALATÓRIA

APBOLUS $=$ IMEDIATAMENTE APÓS O TÉRMINO DO BOLUS

15 BOLUS = 15 MINUTOS APÓS BOLUS

30 BOLUS $=30$ MINUTOS APÓS BOLUS

45 BOLUS $=45$ MINUTOS APÓS BOLUS

75 BOLUS $=75$ MINUTOS APÓS BOLUS 
Tabela 21 - Valores individuais, média de desvio padrão do excesso de base no sangue arterial (mmol/l) dos 5 animais do grupo lidocaína - São Paulo - 2009

\begin{tabular}{ccccccc}
\hline ANIMAIS & 20 INAL & APBOLUS & 15 BOLUS & 30 BOLUS & 45 BOLUS & 75 BOLUS \\
\hline 1 & 0 & 1 & 1 & 1 & 2 & 2 \\
2 & -3 & -4 & -2 & -5 & 0 & 0 \\
3 & 0 & -1 & 0 & 0 & 1 & 2 \\
4 & -1 & 0 & 2 & 3 & 5 & 5 \\
5 & 2 & 2 & 3 & 3 & 4 & 5 \\
\hline MÉDIA & 0 & 0 & 1 & 0 & 2 & 3 \\
DP & 1,82 & 2,30 & 1,92 & 3,29 & 2,07 & 2,17 \\
\hline
\end{tabular}

20 INAL = 20 MINUTOS APÓS ESTABILIZAÇÃO DA ANESTESIA INALATÓRIA

APBOLUS = IMEDIATAMENTE APÓS O TÉRMINO DO BOLUS

15 BOLUS $=15$ MINUTOS APÓS BOLUS

30 BOLUS $=30$ MINUTOS APÓS BOLUS

45 BOLUS $=45$ MINUTOS APÓS BOLUS

75 BOLUS $=75$ MINUTOS APÓS BOLUS

Tabela 22 - Valores individuais, média de desvio padrão da temperatura $\left({ }^{\circ} \mathrm{C}\right)$ dos 5 animais do grupo lidocaína - São Paulo - 2009

\begin{tabular}{ccccccc}
\hline ANIMAIS & 20 INAL & APBOLUS & 15 BOLUS & 30 BOLUS & 45 BOLUS & 75 BOLUS \\
\hline 1 & 37,70 & 37,70 & 37,90 & 37,60 & 37,60 & 37,40 \\
2 & 36,90 & 36,70 & 37,10 & 36,40 & 36,20 & 36,00 \\
3 & 37,00 & 36,30 & 36,30 & 36,10 & 36,00 & 35,70 \\
4 & 37,40 & 37,10 & 36,90 & 36,50 & 36,00 & 36,20 \\
5 & 37,10 & 37,00 & 37,10 & 36,70 & 36,50 & 36,30 \\
\hline MÉDIA & 37,20 & 37,00 & 37,10 & 36,70 & 36,50 & 36,30 \\
DP & 0,33 & 0,52 & 0,57 & 0,57 & 0,67 & 0,65 \\
\hline
\end{tabular}

20 INAL = 20 MINUTOS APÓS ESTABILIZAÇÃO DA ANESTESIA INALATÓRIA APBOLUS $=$ IMEDIATAMENTE APÓS O TÉRMINO DO BOLUS

15 BOLUS $=15$ MINUTOS APÓS BOLUS 30 BOLUS $=30$ MINUTOS APÓS BOLUS 45 BOLUS $=45$ MINUTOS APÓS BOLUS 75 BOLUS $=75$ MINUTOS APÓS BOLUS 


\section{APÊNDICE B- TABELAS RELACIONADAS AO GRUPO QUETAMINA (GQ)}

Tabela 23 - Valores individuais, média e desvio-padrão do peso $(\mathrm{kg})$, débito urinário (ml) e taxa de infusão de fluido Ringer com Lactato de Sódio (I), dos 5 animais do grupo quetamina - São Paulo - 2009

\begin{tabular}{cccc}
\hline ANIMAIS & $\begin{array}{c}\text { PESO } \\
(\mathrm{Kg})\end{array}$ & $\begin{array}{c}\text { DU } \\
(\mathrm{ml})\end{array}$ & $\begin{array}{c}\text { FLUIRO } \\
(\mathrm{L})\end{array}$ \\
\hline 1 & 530 & 1000 & 11 \\
2 & 600 & 1200 & 11 \\
3 & 567 & 0 & 9 \\
4 & 560 & 3,900 & 9 \\
5 & 470 & 1000 & 7 \\
\hline MÉDIA & 549,25 & 1420 & 11,6 \\
DP & 55,64 & 1463,56 & 5,98 \\
\hline
\end{tabular}

DU = DÉBITO URINÁRIA

\section{Parâmetros Hemodinâmicos Diretos}

Tabela 24 - Valores individuais, média de desvio-padrão da freqüência cardíaca (bpm) dos 5 animais do grupo quetamina - São Paulo - 2009

\begin{tabular}{ccccccc}
\hline ANIMAIS & 20 INAL & APBOLUS & 15 BOLUS & 30 BOLUS & 45 BOLUS & 75 BOLUS \\
\hline 1 & 47 & 45 & 49 & 53 & 54 & 53 \\
2 & 43 & 32 & 30 & 34 & 33 & 34 \\
3 & 45 & 42 & 48 & 46 & 43 & 47 \\
4 & 35 & 34 & 38 & 38 & 38 & 38 \\
5 & 46 & 54 & 54 & 50 & 44 & 47 \\
\hline MÉDIA & 43,2 & 41,4 & 43,8 & 44,2 & 42,4 & 43,8 \\
DP & 4,82 & 8,88 & 9,65 & 8,01 & 7,83 & 7,66 \\
\hline
\end{tabular}

20 INAL = 20 MINUTOS APÓS ESTABILIZAÇÃO DA ANESTESIA INALATÓRIA

APBOLUS = IMEDIATAMENTE APÓS O TÉRMINO DO BOLUS

15 BOLUS $=15$ MINUTOS APÓS BOLUS

30 BOLUS $=30$ MINUTOS APÓS BOLUS

45 BOLUS $=45$ MINUTOS APÓS BOLUS

75 BOLUS = 75 MINUTOS APÓS BOLU 
Tabela 25 - Valores individuais, média de desvio-padrão da pressão arterial sistólica ( $\mathrm{mmHg}$ ) dos 5 animais do grupo quetamina - São Paulo - 2009

\begin{tabular}{ccccccc}
\hline ANIMAIS & 20 INAL & APBOLUS & 15 BOLUS & 30 BOLUS & 45 BOLUS & 75 BOLUS \\
\hline 1 & 59 & 73 & 80 & 77 & 81 & 89 \\
2 & 53 & 84 & 95 & 113 & 102 & 85 \\
3 & 68 & 82 & 92 & 81 & 69 & 103 \\
4 & 83 & 91 & 95 & 100 & 103 & 129 \\
5 & 70 & 65 & 71 & 80 & 77 & 99 \\
\hline MÉDIA & 66,60 & 79,00 & 86,60 & 90,20 & 86,40 & 101,00 \\
DP & 11,46 & 10,12 & 10,69 & 15,64 & 15,32 & 17,26 \\
\hline
\end{tabular}

20 INAL = 20 MINUTOS APÓS ESTABILIZAÇÃO DA ANESTESIA INALATÓRIA

APBOLUS = IMEDIATAMENTE APÓS O TÉRMINO DO BOLUS

15 BOLUS = 15 MINUTOS APÓS BOLUS

30 BOLUS $=30$ MINUTOS APÓS BOLUS

45 BOLUS $=45$ MINUTOS APÓS BOLUS

75 BOLUS = 75 MINUTOS APÓS BOLUS

Tabela 26 - Valores individuais, média de desvio-padrão da pressão arterial média (mmHg) dos 5 animais do grupo quetamina - São Paulo - 2009

\begin{tabular}{ccccccc}
\hline ANIMAIS & 20 INAL & APBOLUS & 15 BOLUS & 30 BOLUS & 45 BOLUS & 75 BOLUS \\
\hline 1 & 41 & 53 & 61 & 57 & 64 & 68 \\
2 & 45 & 65 & 76 & 87 & 86 & 66 \\
3 & 59 & 71 & 82 & 68 & 60 & 95 \\
4 & 62 & 71 & 78 & 83 & 91 & 111 \\
5 & 54 & 50 & 55 & 64 & 60 & 82 \\
\hline MÉDIA & 52,2 & 62 & 70,4 & 71,8 & 72,2 & 84,4 \\
DP & 8,98 & 9,95 & 11,72 & 12,76 & 15,07 & 18,93 \\
\hline
\end{tabular}

20 INAL = 20 MINUTOS APÓS ESTABILIZAÇÃO DA ANESTESIA INALATÓRIA

APBOLUS $=$ IMEDIATAMENTE APÓS O TÉRMINO DO BOLUS

15 BOLUS $=15$ MINUTOS APÓS BOLUS

30 BOLUS $=30$ MINUTOS APÓS BOLUS

45 BOLUS $=45$ MINUTOS APÓS BOLUS

75 BOLUS $=75$ MINUTOS APÓS BOLUS 
Tabela 29 - Valores individuais, média de desvio-padrão da saturação arterial de $\mathrm{O}_{2}(\%)$ dos 5 animais do grupo quetamina - São Paulo - 2009

\begin{tabular}{ccccccc}
\hline ANIMAIS & 20 INAL & APBOLUS & 15 BOLUS & 30 BOLUS & 45 BOLUS & 75 BOLUS \\
\hline 1 & 100 & 100 & 100 & 100 & 100 & 100 \\
2 & 100 & 100 & 100 & 100 & 100 & 100 \\
3 & 93 & 98 & 99 & 99 & 99 & 99 \\
4 & 100 & 100 & 100 & 100 & 100 & 100 \\
5 & 100 & 100 & 100 & 100 & 100 & 100 \\
\hline MÉDIA & 98,60 & 99,60 & 99,80 & 99,80 & 99,80 & 99,80 \\
DP & 3,13 & 0,89 & 0,45 & 0,45 & 0,45 & 0,45 \\
\hline
\end{tabular}

20 INAL = 20 MINUTOS APÓS ESTABILIZAÇÃO DA ANESTESIA INALATÓRIA

APBOLUS = IMEDIATAMENTE APÓS O TÉRMINO DO BOLUS

15 BOLUS $=15$ MINUTOS APÓS BOLUS

30 BOLUS $=30$ MINUTOS APÓS BOLUS

45 BOLUS $=45$ MINUTOS APÓS BOLUS

75 BOLUS $=75$ MINUTOS APÓS BOLUS

Tabela 30 - Valores individuais, média de desvio- padrão da pressão parcial de $\mathrm{CO}_{2}$ no sangue arterial $(\mathrm{mmHg})$ dos 5 animais do grupo quetamina - São Paulo 2009

\begin{tabular}{ccccccc}
\hline ANIMAIS & 20 INAL & APBOLUS & 15 BOLUS & 30 BOLUS & 45 BOLUS & 75 BOLUS \\
\hline 1 & 49 & 50 & 45 & 57 & 50 & 49 \\
2 & 50 & 50 & 56 & 51 & 50 & 56 \\
3 & 49 & 49 & 48 & 50 & 49 & 49 \\
4 & 50 & 44 & 41 & 41 & 46 & 40 \\
5 & 48 & 53 & 48 & 51 & 48 & 49 \\
\hline MÉDIA & 49,25 & 49,25 & 47,50 & 50,00 & 48,50 & 48,50 \\
DP & 0,83 & 3,27 & 5,50 & 5,74 & 1,66 & 5,68 \\
\hline
\end{tabular}

20 INAL = 20 MINUTOS APÓS ESTABILIZAÇÃO DA ANESTESIA INALATÓRIA

APBOLUS = IMEDIATAMENTE APÓS O TÉRMINO DO BOLUS

15 BOLUS = 15 MINUTOS APÓS BOLUS

30 BOLUS $=30$ MINUTOS APÓS BOLUS

45 BOLUS $=45$ MINUTOS APÓS BOLUS

75 BOLUS $=75$ MINUTOS APÓS BOLUS 
Tabela 31 - Valores individuais, média de desvio- padrão da freqüência respiratória (mpm) dos 5 animais do grupo quetamina - São Paulo - 2009

\begin{tabular}{ccccccc}
\hline ANIMAIS & 20 INAL & APBOLUS & 15 BOLUS & 30 BOLUS & 45 BOLUS & 75 BOLUS \\
\hline 1 & 10 & 10 & 8 & 8 & 8 & 8 \\
2 & 10 & 10 & 10 & 10 & 10 & 10 \\
3 & 10 & 10 & 10 & 10 & 10 & 10 \\
4 & 12 & 8 & 8 & 8 & 8 & 8 \\
5 & 10 & 10 & 10 & 10 & 10 & 10 \\
\hline MÉDIA & 10,40 & 9,60 & 9,20 & 9,20 & 9,20 & 9,20 \\
DP & 0,89 & 0,89 & 1,10 & 1,10 & 1,10 & 1,10 \\
\hline
\end{tabular}

20 INAL = 20 MINUTOS APÓS ESTABILIZAÇÃO DA ANESTESIA INALATÓRIA

APBOLUS $=$ IMEDIATAMENTE APÓS O TÉRMINO DO BOLUS

15 BOLUS = 15 MINUTOS APÓS BOLUS

30 BOLUS $=30$ MINUTOS APÓS BOLUS

45 BOLUS $=45$ MINUTOS APÓS BOLUS

75 BOLUS = 75 MINUTOS APÓS BOLUS

Tabela 32 - Valores individuais, média de desvio-padrão da concentração do anestésico inalatório no ar expirado (V\%) dos 5 animais do grupo quetamina - São

Paulo - 2009

\begin{tabular}{ccccccc}
\hline ANIMAIS & 20 INAL & APBOLUS & 15 BOLUS & 30 BOLUS & 45 BOLUS & 75 BOLUS \\
\hline 1 & 1,4 & 1,2 & 1,1 & 1,0 & 1,0 & 1,0 \\
2 & 1,5 & 0,9 & 0,6 & 0,7 & 1,4 & 1,3 \\
3 & 1,6 & 1,2 & 1,0 & 1,1 & 1,0 & 1,0 \\
4 & 1,4 & 1,4 & 1,4 & 1,0 & 0,9 & 0,9 \\
5 & 1,3 & 1,5 & 1,1 & 1,0 & 1,0 & 1,3 \\
\hline MÉDIA & 1,44 & 1,24 & 1,04 & 0,96 & 1,06 & 1,10 \\
DP & 0,11 & 0,23 & 0,29 & 0,15 & 0,19 & 0,19 \\
\hline
\end{tabular}

20 INAL = 20 MINUTOS APÓS ESTABILIZAÇÃO DA ANESTESIA INALATÓRIA

APBOLUS $=$ IMEDIATAMENTE APÓS O TÉRMINO DO BOLUS

15 BOLUS $=15$ MINUTOS APÓS BOLUS

30 BOLUS $=30$ MINUTOS APÓS BOLUS

45 BOLUS $=45$ MINUTOS APÓS BOLUS

75 BOLUS = 75 MINUTOS APÓS BOLUS 
Tabela 33 - Valores individuais, média de desvio- padrão do volume inspiratório (I) dos 5 animais do grupo quetamina - São Paulo - 2009

\begin{tabular}{ccccccc}
\hline ANIMAIS & 20 INAL & APBOLUS & 15 BOLUS & 30 BOLUS & 45 BOLUS & 75 BOLUS \\
\hline 1 & 10 & 10 & 10 & 10 & 10 & 10 \\
2 & 9 & 9 & 9 & 9 & 9 & 9 \\
3 & 8 & 8 & 8 & 8 & 8 & 8 \\
4 & 8 & 10 & 10 & 10 & 10 & 10 \\
5 & 8 & 8 & 8 & 8 & 8 & 8 \\
\hline MÉDIA & 8,60 & 9,00 & 9,00 & 9,00 & 9,00 & 9,00 \\
DP & 0,89 & 1,00 & 1,00 & 1,00 & 1,00 & 1,00 \\
\hline
\end{tabular}

20 INAL = 20 MINUTOS APÓS ESTABILIZAÇÃO DA ANESTESIA INALATÓRIA

APBOLUS = IMEDIATAMENTE APÓS O TÉRMINO DO BOLUS

15 BOLUS = 15 MINUTOS APÓS BOLUS

30 BOLUS $=30$ MINUTOS APÓS BOLUS

45 BOLUS = 45 MINUTOS APÓS BOLUS

75 BOLUS $=75$ MINUTOS APÓS BOLUS

Tabela 34 - Valores individuais, média de desvio- padrão do volume expiratório (I)

\begin{tabular}{ccccccc}
\hline ANIMAIS & 20 INAL & APBOLUS & 15 BOLUS & 30 BOLUS & 45 BOLUS & 75 BOLUS \\
\hline 1 & 7,82 & 7,73 & 10,0 & 10,0 & 10,0 & 9,93 \\
2 & 8,75 & 8,72 & 8,68 & 8,63 & 8,76 & 8,76 \\
3 & 6,95 & 6,97 & 7,01 & 6,86 & 6,38 & 7,20 \\
4 & 5,70 & 8,56 & 8,59 & 8,58 & 8,43 & 8,18 \\
5 & 7,13 & 6,90 & 6,85 & 6,76 & 6,69 & 6,70 \\
\hline MÉDIA & 7,27 & 7,78 & 8,23 & 8,17 & 8,05 & 8,15 \\
DP & 1,13 & 0,86 & 1,31 & 1,36 & 1,51 & 1,28 \\
\hline
\end{tabular}

dos 5 animais do grupo quetamina - São Paulo - 2009

20 INAL = 20 MINUTOS APÓS ESTABILIZAÇÃO DA ANESTESIA INALATÓRIA

APBOLUS = IMEDIATAMENTE APÓS O TÉRMINO DO BOLUS

15 BOLUS $=15$ MINUTOS APÓS BOLUS

30 BOLUS $=30$ MINUTOS APÓS BOLUS

45 BOLUS $=45$ MINUTOS APÓS BOLUS

75 BOLUS $=75$ MINUTOS APÓS BOLUS 
Tabela 35- Valores individuais, média de desvio- padrão do platô $\left(\mathrm{cmH}_{2} \mathrm{O}\right)$ dos 5 animais do grupo quetamina - São Paulo - 2009

\begin{tabular}{ccccccc}
\hline ANIMAIS & 20 INAL & APBOLUS & 15 BOLUS & 30 BOLUS & 45 BOLUS & 75 BOLUS \\
\hline 1 & 19 & 20 & 22 & 22 & 22 & 25 \\
2 & 24 & 21 & 21 & 21 & 22 & 21 \\
3 & 19 & 17 & 20 & 19 & 18 & 17 \\
4 & 19 & 22 & 23 & 21 & 21 & 21 \\
5 & 22 & 20 & 20 & 21 & 21 & 20 \\
\hline MÉDIA & 20,60 & 20,00 & 21,20 & 20,80 & 20,80 & 20,80 \\
DP & 2,30 & 1,87 & 1,30 & 1,10 & 1,64 & 2,86 \\
\hline
\end{tabular}

20 INAL = 20 MINUTOS APÓS ESTABILIZAÇÃO DA ANESTESIA INALATÓRIA

APBOLUS $=$ IMEDIATAMENTE APÓS O TÉRMINO DO BOLUS

15 BOLUS $=15$ MINUTOS APÓS BOLUS

30 BOLUS $=30$ MINUTOS APÓS BOLUS

45 BOLUS $=45$ MINUTOS APÓS BOLUS

75 BOLUS = 75 MINUTOS APÓS BOLUS

Tabela 36 - Valores individuais, média de desvio- padrão da resistência em vias aéreas $\left(\mathrm{cmH}_{2} \mathrm{O} / / / \mathrm{seg}\right)$ dos 5 animais do grupo quetamina - São Paulo - 2009

\begin{tabular}{ccccccc}
\hline ANIMAIS & 20 INAL & APBOLUS & 15 BOLUS & 30 BOLUS & 45 BOLUS & 75 BOLUS \\
\hline 1 & 9 & 9 & 10 & 11 & 10 & 10 \\
2 & 6 & 6 & 6 & 6 & 6 & 6 \\
3 & 7 & 7 & 8 & 7 & 7 & 7 \\
4 & 8 & 9 & 9 & 9 & 9 & 9 \\
5 & 9 & 9 & 8 & 8 & 9 & 9 \\
\hline MÉDIA & 7,80 & 8,00 & 8,20 & 8,20 & 8,20 & 8,20 \\
DP & 1,30 & 1,41 & 1,48 & 1,92 & 1,64 & 1,64 \\
\hline
\end{tabular}

20 INAL = 20 MINUTOS APÓS ESTABILIZAÇÃO DA ANESTESIA INALATÓRIA

APBOLUS = IMEDIATAMENTE APÓS O TÉRMINO DO BOLUS

15 BOLUS = 15 MINUTOS APÓS BOLUS

30 BOLUS $=30$ MINUTOS APÓS BOLUS

45 BOLUS $=45$ MINUTOS APÓS BOLUS

75 BOLUS $=75$ MINUTOS APÓS BOLUS 
Tabela 37 - Valores individuais, média de desvio- padrão da complacência estática das vias aéreas $\left(\mathrm{ml} / \mathrm{cmH}_{2} \mathrm{O}\right)$ dos 5 animais do grupo quetamina - São Paulo - 2009

\begin{tabular}{ccccccc}
\hline ANIMAIS & 20 INAL & APBOLUS & 15 BOLUS & 30 BOLUS & 45 BOLUS & 75 BOLUS \\
\hline 1 & 268 & 269 & 278 & 286 & 278 & 270 \\
2 & 275 & 110 & 96 & 92 & 84 & 85 \\
3 & 155 & 158 & 162 & 160 & 161 & 157 \\
4 & 131 & 160 & 164 & 166 & 169 & 164 \\
5 & 138 & 142 & 149 & 139 & 140 & 140 \\
\hline MÉDIA & 193,40 & 167,80 & 169,80 & 168,60 & 166,40 & 163,20 \\
DP & 71,87 & 60,01 & 66,48 & 71,78 & 70,67 & 67,27 \\
\hline
\end{tabular}

20 INAL = 20 MINUTOS APÓS ESTABILIZAÇÃO DA ANESTESIA INALATÓRIA APBOLUS = IMEDIATAMENTE APÓS O TÉRMINO DO BOLUS

15 BOLUS $=15$ MINUTOS APÓS BOLUS

30 BOLUS $=30$ MINUTOS APÓS BOLUS

45 BOLUS = 45 MINUTOS APÓS BOLUS

75 BOLUS = 75 MINUTOS APÓS BOLUS

Tabela 38 - Valores individuais, média de desvio- padrão da pressão positiva no final da expiração $\left(\mathrm{cmH}_{2} \mathrm{O}\right)$ dos 5 animais do grupo quetamina - São Paulo - 2009

\begin{tabular}{ccccccc}
\hline ANIMAIS & 20 INAL & APBOLUS & 15 BOLUS & 30 BOLUS & 45 BOLUS & 75 BOLUS \\
\hline 1 & 7 & 7 & 7 & 7 & 7 & 7 \\
2 & 7 & 7 & 7 & 7 & 7 & 7 \\
3 & 7 & 7 & 7 & 7 & 7 & 7 \\
4 & 7 & 7 & 7 & 7 & 7 & 7 \\
5 & 7 & 7 & 7 & 7 & 7 & 7 \\
\hline MÉDIA & 7,00 & 7,00 & 7,00 & 7,00 & 7,00 & 7,00 \\
DP & 0,00 & 0,00 & 0,00 & 0,00 & 0,00 & 0,00 \\
\hline
\end{tabular}

20 INAL = 20 MINUTOS APÓS ESTABILIZAÇÃO DA ANESTESIA INALATÓRIA

APBOLUS $=$ IMEDIATAMENTE APÓS O TÉRMINO DO BOLUS

15 BOLUS $=15$ MINUTOS APÓS BOLUS

30 BOLUS $=30$ MINUTOS APÓS BOLUS

45 BOLUS $=45$ MINUTOS APÓS BOLUS

75 BOLUS $=75$ MINUTOS APÓS BOLUS 
Tabela 39 - Valores individuais, média de desvio- padrão da pressão de pico inspiratória $\left(\mathrm{cm}_{\mathrm{H} 2} \mathrm{O}\right)$ dos 5 animais do grupo quetamina - São Paulo - 2009

\begin{tabular}{ccccccc}
\hline ANIMAIS & 20 INAL & APBOLUS & 15 BOLUS & 30 BOLUS & 45 BOLUS & 75 BOLUS \\
\hline 1 & 24 & 24 & 26 & 26 & 26 & 28 \\
2 & 29 & 27 & 27 & 27 & 27 & 27 \\
3 & 25 & 22 & 23 & 23 & 23 & 23 \\
4 & 23 & 25 & 25 & 25 & 25 & 25 \\
5 & 25 & 25 & 25 & 25 & 25 & 25 \\
\hline MÉDIA & 25,20 & 24,60 & 25,20 & 25,20 & 25,20 & 25,60 \\
DP & 2,28 & 1,82 & 1,48 & 1,48 & 1,48 & 1,95 \\
\hline
\end{tabular}

20 INAL = 20 MINUTOS APÓS ESTABILIZAÇÃO DA ANESTESIA INALATÓRIA

APBOLUS $=$ IMEDIATAMENTE APÓS O TÉRMINO DO BOLUS

15 BOLUS $=15$ MINUTOS APÓS BOLUS

30 BOLUS $=30$ MINUTOS APÓS BOLUS

45 BOLUS $=45$ MINUTOS APÓS BOLUS

75 BOLUS = 75 MINUTOS APÓS BOLUS

Tabela 40 - Valores individuais, média de desvio- padrão do $\mathrm{CO}_{2}$ no final da expiração das vias aéreas $(\mathrm{mmHg})$ dos 5 animais do grupo quetamina - São Paulo - 2009

\begin{tabular}{ccccccc}
\hline ANIMAIS & 20 INAL & APBOLUS & 15 BOLUS & 30 BOLUS & 45 BOLUS & 75 BOLUS \\
\hline 1 & 38 & 42 & 37 & 38 & 38 & 38 \\
2 & 38 & 40 & 40 & 41 & 40 & 40 \\
3 & 43 & 44 & 43 & 43 & 43 & 49 \\
4 & 41 & 38 & 38 & 38 & 37 & 38 \\
5 & 36 & 37 & 37 & 36 & 36 & 37 \\
\hline MÉDIA & 39,20 & 40,20 & 39,00 & 39,20 & 38,80 & 40,40 \\
DP & 2,77 & 2,86 & 2,55 & 2,77 & 2,77 & 4,93 \\
\hline
\end{tabular}

20 INAL = 20 MINUTOS APÓS ESTABILIZAÇÃO DA ANESTESIA INALATÓRIA

APBOLUS = IMEDIATAMENTE APÓS O TÉRMINO DO BOLUS

15 BOLUS $=15$ MINUTOS APÓS BOLUS

30 BOLUS = 30 MINUTOS APÓS BOLUS

45 BOLUS $=45$ MINUTOS APÓS BOLUS

75 BOLUS $=75$ MINUTOS APÓS BOLUS 
Tabela 41 - Valores individuais, média de desvio- padrão do pH dos 5 animais do grupo quetamina - São Paulo - 2009

\begin{tabular}{ccccccc}
\hline ANIMAIS & 20 INAL & APBOLUS & 15 BOLUS & 30 BOLUS & 45 BOLUS & 75 BOLUS \\
\hline 1 & 7,30 & 7,30 & 7,33 & 7,31 & 7,31 & 7,31 \\
2 & 7,34 & 7,33 & 7,31 & 7,33 & 7,34 & 7,32 \\
3 & 7,34 & 7,34 & 7,35 & 7,35 & 7,35 & 7,35 \\
4 & 7,32 & 7,35 & 7,35 & 7,34 & 7,36 & 7,35 \\
5 & 7,41 & 7,37 & 7,40 & 7,40 & 7,40 & 7,40 \\
\hline MÉDIA & 7,34 & 7,34 & 7,35 & 7,35 & 7,35 & 7,35 \\
DP & 0,04 & 0,03 & 0,03 & 0,03 & 0,03 & 0,04 \\
\hline
\end{tabular}

20 INAL = 20 MINUTOS APÓS ESTABILIZAÇÃO DA ANESTESIA INALATÓRIA

APBOLUS = IMEDIATAMENTE APÓS O TÉRMINO DO BOLUS

15 BOLUS = 15 MINUTOS APÓS BOLUS

30 BOLUS $=30$ MINUTOS APÓS BOLUS

45 BOLUS $=45$ MINUTOS APÓS BOLUS

75 BOLUS = 75 MINUTOS APÓS BOLUS

Tabela 42 - Valores individuais, média de desvio- padrão do bicarbonato no sangue arterial (mmol/l) dos 5 animais do grupo quetamina - São Paulo - 2009

\begin{tabular}{ccccccc}
\hline ANIMAIS & 20 INAL & APBOLUS & 15 BOLUS & 30 BOLUS & 45 BOLUS & 75 BOLUS \\
\hline 1 & 23 & 24 & 23 & 23 & 24 & 24 \\
2 & 26 & 26 & 27 & 26 & 26 & 28 \\
3 & 28 & 26 & 27 & 26 & 24 & 28 \\
4 & 24 & 23 & 22 & 21 & 21 & 21 \\
5 & 29 & 29 & 29 & 30 & 29 & 30 \\
\hline MÉDIA & 26,00 & 25,60 & 25,60 & 25,20 & 24,80 & 26,20 \\
DP & 2,55 & 2,30 & 2,97 & 3,42 & 2,95 & 3,63 \\
\hline
\end{tabular}

20 INAL = 20 MINUTOS APÓS ESTABILIZAÇÃO DA ANESTESIA INALATÓRIA APBOLUS $=$ IMEDIATAMENTE APÓS O TÉRMINO DO BOLUS

15 BOLUS $=15$ MINUTOS APÓS BOLUS 30 BOLUS $=30$ MINUTOS APÓS BOLUS 45 BOLUS $=45$ MINUTOS APÓS BOLUS 75 BOLUS $=75$ MINUTOS APÓS BOLUS 
Tabela 43 - Valores individuais, média de desvio- padrão do excesso de base no sangue arterial (mmol/l) dos 5 animais do grupo quetamina - São Paulo - 2009

\begin{tabular}{ccccccc}
\hline ANIMAIS & 20 INAL & APBOLUS & 15 BOLUS & 30 BOLUS & 45 BOLUS & 75 BOLUS \\
\hline 1 & -3 & -2 & -3 & -3 & -2 & -2 \\
2 & 1 & 0 & 1 & 0 & 0 & 2 \\
3 & -1 & -2 & -1 & -1 & -2 & -1 \\
4 & -1 & -2 & -3 & -3 & 0 & -3 \\
5 & 4 & 4 & 4 & 5 & 4 & 5 \\
\hline MÉDIA & 0,00 & $-0,40$ & $-0,40$ & $-0,40$ & 0,00 & 0,20 \\
DP & 2,65 & 2,61 & 2,97 & 3,29 & 2,45 & 3,27 \\
\hline
\end{tabular}

20 INAL = 20 MINUTOS APÓS ESTABILIZAÇÃO DA ANESTESIA INALATÓRIA APBOLUS $=$ IMEDIATAMENTE APÓS O TÉRMINO DO BOLUS

15 BOLUS = 15 MINUTOS APÓS BOLUS

30 BOLUS $=30$ MINUTOS APÓS BOLUS

45 BOLUS $=45$ MINUTOS APÓS BOLUS

75 BOLUS = 75 MINUTOS APÓS BOLUS

Tabela 44 - Valores individuais, média de desvio- padrão da temperatura $\left({ }^{\circ} \mathrm{C}\right)$ dos 5 animais do grupo quetamina - São Paulo - 2009

\begin{tabular}{ccccccc}
\hline ANIMAIS & 20 INAL & APBOLUS & 15 BOLUS & 30 BOLUS & 45 BOLUS & 75 BOLUS \\
\hline 1 & 38,0 & 37.8 & 38,0 & 38,0 & 38.2 & 38,2 \\
2 & 37,8 & 37,3 & 37,2 & 37,0 & 37,1 & 37,0 \\
3 & 37,6 & 37,7 & 37,7 & 37,4 & 37,1 & 37,2 \\
4 & 38,3 & 38,2 & 37,8 & 38,0 & 37,8 & 37,7 \\
5 & 38,2 & 38,2 & 38,3 & 38,2 & 38,1 & 37,9 \\
\hline MÉDIA & 37,98 & 37,85 & 37,80 & 37,72 & 37,53 & 37,60 \\
DP & 0,29 & 0,44 & 0,41 & 0,50 & 0,51 & 0,49 \\
\hline
\end{tabular}

20 INAL = 20 MINUTOS APÓS ESTABILIZAÇÃO DA ANESTESIA INALATÓRIA APBOLUS = IMEDIATAMENTE APÓS O TÉRMINO DO BOLUS

15 BOLUS $=15$ MINUTOS APÓS BOLUS

30 BOLUS $=30$ MINUTOS APÓS BOLUS

45 BOLUS $=45$ MINUTOS APÓS BOLUS

75 BOLUS $=75$ MINUTOS APÓS BOLUS 


\section{APÊNDICE C- TABELAS RELACIONADAS AO GRUPO FENTANIL (GF)}

Tabela 45 - Valores individuais, média e desvio-padrão do peso $(\mathrm{kg})$, débito urinário (ml) e taxa de infusão de fluido Ringer com Lactato de Sódio (l), dos 5 animais do grupo fentanil - São Paulo - 2009

\begin{tabular}{cccc}
\hline ANIMAIS & $\begin{array}{c}\text { PESO } \\
(\mathrm{Kg})\end{array}$ & $\begin{array}{c}\text { DU } \\
(\mathrm{ml})\end{array}$ & $\begin{array}{c}\text { FLUIDO } \\
(\mathrm{L})\end{array}$ \\
\hline 1 & 368 & 8000 & 8 \\
2 & 368 & 3000 & 9 \\
3 & 450 & 4000 & 5 \\
4 & 390 & 4000 & 10 \\
5 & 515 & 0 & 8 \\
MÉDIA & 418,2 & 3800 & 8 \\
DP & 63,67 & 2863,56 & 1,87 \\
\hline
\end{tabular}

\section{Parâmetros Hemodinâmicos Diretos}

Tabela 46 - Valores individuais, média de desvio- padrão da freqüência cardíaca (bpm) dos 5 animais do grupo fentanil - São Paulo - 2009

\begin{tabular}{ccccccc}
\hline ANIMAIS & 20 INAL & APBOLUS & 15 BOLUS & 30 BOLUS & 45 BOLUS & 75 BOLUS \\
\hline 1 & 44 & 44 & 47 & 48 & 51 & 50 \\
2 & 36 & 33 & 34 & 45 & 47 & 45 \\
3 & 50 & 42 & 43 & 42 & 42 & 42 \\
4 & 44 & 50 & 52 & 50 & 45 & 41 \\
5 & 51 & 51 & 46 & 42 & 40 & 37 \\
\hline MÉDIA & 45,0 & 44,0 & 44,4 & 45,4 & 45,0 & 43,0 \\
DP & 6,00 & 7,25 & 6,66 & 3,58 & 4,30 & 4,85 \\
\hline
\end{tabular}

20 INAL = 20 MINUTOS APÓS ESTABILIZAÇÃO DA ANESTESIA INALATÓRIA

APBOLUS = IMEDIATAMENTE APÓS O TÉRMINO DO BOLUS

15 BOLUS $=15$ MINUTOS APÓS BOLUS

30 BOLUS $=30$ MINUTOS APÓS BOLUS

45 BOLUS $=45$ MINUTOS APÓS BOLUS

75 BOLUS $=75$ MINUTOS APÓS BOLUS 
Tabela 47 - Valores individuais, média de desvio- padrão da pressão arterial sistólica (bpm) dos 5 animais do grupo fentanil - São Paulo - 2009

\begin{tabular}{ccccccc}
\hline ANIMAIS & 20 INAL & APBOLUS & 15 BOLUS & 30 BOLUS & 45 BOLUS & 75 BOLUS \\
\hline 1 & 81 & 103 & 71 & 88 & 91 & 87 \\
2 & 73 & 87 & 107 & 98 & 99 & 88 \\
3 & 58 & 73 & 92 & 87 & 88 & 93 \\
4 & 99 & 78 & 98 & 92 & 96 & 112 \\
5 & 49 & 55 & 79 & 76 & 86 & 76 \\
\hline MÉDIA & 72,0 & 79,2 & 89,4 & 88,2 & 92,0 & 91,2 \\
DP & 19,60 & 17,70 & 14,47 & 8,07 & 5,43 & 13,18 \\
\hline
\end{tabular}

20 INAL = 20 MINUTOS APÓS ESTABILIZAÇÃO DA ANESTESIA INALATÓRIA

APBOLUS = IMEDIATAMENTE APÓS O TÉRMINO DO BOLUS

15 BOLUS = 15 MINUTOS APÓS BOLUS

30 BOLUS $=30$ MINUTOS APÓS BOLUS

45 BOLUS $=45$ MINUTOS APÓS BOLUS

75 BOLUS = 75 MINUTOS APÓS BOLUS

Tabela 48 - Valores individuais, média de desvio- padrão da pressão arterial média (mmHg) dos 5 animais do grupo fentanil - São Paulo - 2009

\begin{tabular}{ccccccc}
\hline ANIMAIS & 20 INAL & APBOLUS & 15 BOLUS & 30 BOLUS & 45 BOLUS & 75 BOLUS \\
\hline 1 & 53 & 67 & 62 & 57 & 69 & 56 \\
2 & 50 & 60 & 76 & 71 & 66 & 63 \\
3 & 41 & 51 & 64 & 60 & 61 & 65 \\
4 & 70 & 55 & 72 & 67 & 72 & 83 \\
5 & 40 & 45 & 65 & 61 & 67 & 52 \\
\hline MÉDIA & 50,8 & 55,6 & 67,8 & 63,2 & 67,0 & 63,8 \\
DP & 12,11 & 8,41 & 5,93 & 5,67 & 4,06 & 11,95 \\
\hline
\end{tabular}

20 INAL = 20 MINUTOS APÓS ESTABILIZAÇÃO DA ANESTESIA INALATÓRIA

APBOLUS = IMEDIATAMENTE APÓS O TÉRMINO DO BOLUS

15 BOLUS $=15$ MINUTOS APÓS BOLUS

30 BOLUS $=30$ MINUTOS APÓS BOLUS

45 BOLUS $=45$ MINUTOS APÓS BOLUS

75 BOLUS = 75 MINUTOS APÓS BOLUS 
Tabela 49 - Valores individuais, média de desvio- padrão da pressão arterial diastólica (mmHg) dos 5 animais do grupo fentanil - São Paulo - 2009

\begin{tabular}{ccccccc}
\hline ANIMAIS & 20 INAL & APBOLUS & 15 BOLUS & 30 BOLUS & 45 BOLUS & 75 BOLUS \\
\hline 1 & 39 & 49 & 56 & 43 & 52 & 43 \\
2 & 37 & 46 & 57 & 53 & 48 & 46 \\
3 & 32 & 42 & 51 & 48 & 48 & 52 \\
4 & 57 & 44 & 59 & 53 & 58 & 65 \\
5 & 31 & 39 & 56 & 51 & 56 & 41 \\
\hline MÉDIA & 39,2 & 44,0 & 55,8 & 49,6 & 52,4 & 49,4 \\
DP & 10,50 & 3,81 & 2,95 & 4,22 & 4,56 & 9,66 \\
\hline
\end{tabular}

20 INAL = 20 MINUTOS APÓS ESTABILIZAÇÃO DA ANESTESIA INALATÓRIA

APBOLUS = IMEDIATAMENTE APÓS O TÉRMINO DO BOLUS

15 BOLUS $=15$ MINUTOS APÓS BOLUS

30 BOLUS $=30$ MINUTOS APÓS BOLUS

45 BOLUS $=45$ MINUTOS APÓS BOLUS

75 BOLUS $=75$ MINUTOS APÓS BOLUS

\section{Parâmetros de Ventilação, Metabólicos e de Temperatura}

Tabela 50 - Valores individuais, média de desvio- padrão da pressão parcial de $\mathrm{O}_{2}$ no sangue arterial $(\mathrm{mmHg})$ dos 5 animais do grupo fentanil - São Paulo - 2009

\begin{tabular}{ccccccc}
\hline ANIMAIS & 20 INAL & APBOLUS & 15 BOLUS & 30 BOLUS & 45 BOLUS & 75 BOLUS \\
\hline 1 & 284 & 228 & 135 & 173 & 191 & 204 \\
2 & 274 & 232 & 232 & 260 & 286 & 323 \\
3 & 312 & 310 & 312 & 308 & 334 & 312 \\
4 & 315 & 359 & 342 & 355 & 345 & 255 \\
5 & 315 & 329 & 292 & 347 & 322 & 389 \\
\hline MÉDIA & 300 & 292 & 263 & 289 & 296 & 297 \\
DP & 19,53 & 58,90 & 81,90 & 74,80 & 62,54 & 70,32 \\
\hline
\end{tabular}

20 INAL = 20 MINUTOS APÓS ESTABILIZAÇÃO DA ANESTESIA INALATÓRIA APBOLUS = IMEDIATAMENTE APÓS O TÉRMINO DO BOLUS

15 BOLUS $=15$ MINUTOS APÓS BOLUS 30 BOLUS $=30$ MINUTOS APÓS BOLUS 45 BOLUS $=45$ MINUTOS APÓS BOLUS 75 BOLUS $=75$ MINUTOS APÓS BOLUS 
Tabela 51 - Valores individuais, média de desvio- padrão da saturação arterial de $\mathrm{O}_{2}(\%)$ dos 5 animais do grupo fentanil- São Paulo - 2009

\begin{tabular}{ccccccc}
\hline ANIMAIS & 20 INAL & APBOLUS & 15 BOLUS & 30 BOLUS & 45 BOLUS & 75 BOLUS \\
\hline 1 & 100 & 100 & 99 & 99 & 99 & 100 \\
2 & 100 & 100 & 100 & 100 & 100 & 100 \\
3 & 100 & 99 & 100 & 100 & 100 & 100 \\
4 & 100 & 100 & 100 & 100 & 100 & 100 \\
5 & 100 & 100 & 100 & 100 & 100 & 100 \\
\hline MÉDIA & 100 & 100 & 100 & 100 & 100 & 100 \\
DP & 0,00 & 0,45 & 0,45 & 0,45 & 0,45 & 0,00 \\
\hline
\end{tabular}

20 INAL = 20 MINUTOS APÓS ESTABILIZAÇÃO DA ANESTESIA INALATÓRIA

APBOLUS $=$ IMEDIATAMENTE APÓS O TÉRMINO DO BOLUS

15 BOLUS = 15 MINUTOS APÓS BOLUS

30 BOLUS $=30$ MINUTOS APÓS BOLUS

45 BOLUS $=45$ MINUTOS APÓS BOLUS

75 BOLUS $=75$ MINUTOS APÓS BOLUS

Tabela 52 - Valores individuais, média de desvio- padrão da pressão parcial de $\mathrm{CO}_{2}$ no sangue arterial $(\mathrm{mmHg})$ dos 5 animais do grupo fentanil- São Paulo - 2009

\begin{tabular}{ccccccc}
\hline ANIMAIS & 20 INAL & APBOLUS & 15 BOLUS & 30 BOLUS & 45 BOLUS & 75 BOLUS \\
\hline 1 & 51 & 47 & 52 & 53 & 53 & 49 \\
2 & 39 & 46 & 45 & 50 & 46 & 38 \\
3 & 45 & 44 & 47 & 47 & 47 & 44 \\
4 & 48 & 48 & 46 & 48 & 46 & 44 \\
5 & 43 & 46 & 31 & 42 & 36 & 45 \\
\hline MÉDIA & 45,2 & 46,2 & 44,2 & 48,0 & 45,6 & 44,0 \\
DP & 4,60 & 1,48 & 7,85 & 4,06 & 6,11 & 3,94 \\
\hline
\end{tabular}

20 INAL = 20 MINUTOS APÓS ESTABILIZAÇÃO DA ANESTESIA INALATÓRIA

APBOLUS $=$ IMEDIATAMENTE APÓS O TÉRMINO DO BOLUS

15 BOLUS $=15$ MINUTOS APÓS BOLUS

30 BOLUS $=30$ MINUTOS APÓS BOLUS

45 BOLUS $=45$ MINUTOS APÓS BOLUS

75 BOLUS $=75$ MINUTOS APÓS BOLUS 
Tabela 53 - Valores individuais, média de desvio- padrão da freqüência respiratória (bpm) dos 5 animais do grupo fentanil- São Paulo - 2009

\begin{tabular}{ccccccc}
\hline ANIMAIS & 20 INAL & APBOLUS & 15 BOLUS & 30 BOLUS & 45 BOLUS & 75 BOLUS \\
\hline 1 & 8 & 8 & 8 & 8 & 8 & 8 \\
2 & 8 & 8 & 8 & 8 & 8 & 8 \\
3 & 8 & 8 & 8 & 8 & 8 & 8 \\
4 & 10 & 10 & 10 & 10 & 10 & 10 \\
5 & 8 & 8 & 8 & 8 & 8 & 8 \\
\hline MÉDIA & 8,4 & 8,4 & 8,4 & 8,4 & 8,4 & 8,4 \\
DP & 0,89 & 0,89 & 0,89 & 0,89 & 0,89 & 0,89 \\
\hline
\end{tabular}

20 INAL = 20 MINUTOS APÓS ESTABILIZAÇÃO DA ANESTESIA INALATÓRIA

APBOLUS = IMEDIATAMENTE APÓS O TÉRMINO DO BOLUS

15 BOLUS = 15 MINUTOS APÓS BOLUS

30 BOLUS $=30$ MINUTOS APÓS BOLUS

45 BOLUS $=45$ MINUTOS APÓS BOLUS

75 BOLUS $=75$ MINUTOS APÓS BOLUS

Tabela 54 - Valores individuais, média de desvio- padrão da concentração de anestésico inalatório no ar expirado (V\%) dos 5 animais do grupo fentanil - São

Paulo - 2009

\begin{tabular}{ccccccc}
\hline ANIMAIS & 20 INAL & APBOLUS & 15 BOLUS & 30 BOLUS & 45 BOLUS & 75 BOLUS \\
\hline 1 & 1,4 & 1,3 & 1,1 & 0,9 & 1,0 & 1,4 \\
2 & 1,3 & 1,2 & 1,0 & 1,0 & 1,0 & 1,6 \\
3 & 1,3 & 1,3 & 1,2 & 0,9 & 1,0 & 1,2 \\
4 & 1,3 & 1,2 & 0,9 & 1,0 & 1,0 & 1,4 \\
5 & 1,3 & 1,0 & 1,0 & 1,0 & 1,0 & 1,3 \\
\hline MÉDIA & 1,32 & 1,2 & 1,04 & 0,96 & 1 & 1,38 \\
DP & 0,04 & 0,12 & 0,11 & 0,05 & 0,00 & 0,15 \\
\hline
\end{tabular}

20 INAL = 20 MINUTOS APÓS ESTABILIZAÇÃO DA ANESTESIA INALATÓRIA

APBOLUS $=$ IMEDIATAMENTE APÓS O TÉRMINO DO BOLUS

15 BOLUS $=15$ MINUTOS APÓS BOLUS

30 BOLUS $=30$ MINUTOS APÓS BOLUS

45 BOLUS = 45 MINUTOS APÓS BOLUS

75 BOLUS $=75$ MINUTOS APÓS BOLUS 
Tabela 55 - Valores individuais, média de desvio- padrão do volume inspiratório (I) dos 5 animais do grupo fentanil - São Paulo - 2009

\begin{tabular}{ccccccc}
\hline ANIMAIS & 20 INAL & APBOLUS & 15 BOLUS & 30 BOLUS & 45 BOLUS & 75 BOLUS \\
\hline 1 & 7 & 8 & 8 & 8 & 8 & 8 \\
2 & 7 & 7 & 7 & 7 & 8 & 8 \\
3 & 9 & 9 & 10 & 10 & 10 & 10 \\
4 & 7 & 7 & 7 & 7 & 7 & 7 \\
5 & 10 & 10 & 10 & 10 & 10 & 10 \\
\hline MÉDIA & 8,0 & 8,2 & 8,4 & 8,4 & 8,6 & 8,6 \\
DP & 1,41 & 1,30 & 1,52 & 1,52 & 1,34 & 1,34 \\
\hline
\end{tabular}

20 INAL = 20 MINUTOS APÓS ESTABILIZAÇÃO DA ANESTESIA INALATÓRIA APBOLUS = IMEDIATAMENTE APÓS O TÉRMINO DO BOLUS

15 BOLUS $=15$ MINUTOS APÓS BOLUS

30 BOLUS $=30$ MINUTOS APÓS BOLUS

45 BOLUS $=45$ MINUTOS APÓS BOLUS

75 BOLUS $=75$ MINUTOS APÓS BOLUS

Tabela 56 - Valores individuais, média de desvio- padrão do volume expiratório (I) dos 5 animais do grupo fentanil - São Paulo - 2009

\begin{tabular}{ccccccc}
\hline ANIMAIS & 20 INAL & APBOLUS & 15 BOLUS & 30 BOLUS & 45 BOLUS & 75 BOLUS \\
\hline 1 & 5,61 & 6,17 & 5,94 & 5,85 & 5,98 & 5,9 \\
2 & 5,34 & 5,44 & 5,49 & 5,81 & 6,06 & 6,06 \\
3 & 7,38 & 7,51 & 7,51 & 7,61 & 7,61 & 7,15 \\
4 & 5,5 & 5,29 & 5,3 & 5,25 & 5,46 & 5,22 \\
5 & 7,93 & 8,4 & 8,17 & 8,2 & 8,3 & 8,25 \\
\hline MÉDIA & 6,4 & 6,6 & 6,5 & 6,5 & 6,7 & 6,5 \\
DP & 1,21 & 1,35 & 1,28 & 1,28 & 1,21 & 1,19 \\
\hline
\end{tabular}

20 INAL = 20 MINUTOS APÓS ESTABILIZAÇÃO DA ANESTESIA INALATÓRIA

APBOLUS = IMEDIATAMENTE APÓS O TÉRMINO DO BOLUS

15 BOLUS $=15$ MINUTOS APÓS BOLUS

30 BOLUS $=30$ MINUTOS APÓS BOLUS

45 BOLUS $=45$ MINUTOS APÓS BOLUS

75 BOLUS $=75$ MINUTOS APÓS BOLUs 
Tabela 57 - Valores individuais, média de desvio- padrão do platô $\left(\mathrm{cmH}_{2} \mathrm{O}\right)$ dos 5 animais do grupo fentanil - São Paulo - 2009

\begin{tabular}{ccccccc}
\hline ANIMAIS & 20 INAL & APBOLUS & 15 BOLUS & 30 BOLUS & 45 BOLUS & 75 BOLUS \\
\hline 1 & 25 & 27 & 26 & 26 & 27 & 30 \\
2 & 25 & 25 & 25 & 25 & 25 & 25 \\
3 & 27 & 28 & 27 & 27 & 28 & 27 \\
4 & 29 & 26 & 26 & 25 & 25 & 27 \\
5 & 26 & 26 & 26 & 26 & 26 & 26 \\
\hline MÉDIA & 26,4 & 26,4 & 26,00 & 25,8 & 26,2 & 27 \\
DP & 1,67 & 1,14 & 0,71 & 0,84 & 1,30 & 1,87 \\
\hline
\end{tabular}

20 INAL = 20 MINUTOS APÓS ESTABILIZAÇÃO DA ANESTESIA INALATÓRIA

APBOLUS = IMEDIATAMENTE APÓS O TÉRMINO DO BOLUS

15 BOLUS $=15$ MINUTOS APÓS BOLUS

30 BOLUS $=30$ MINUTOS APÓS BOLUS

45 BOLUS $=45$ MINUTOS APÓS BOLUS

75 BOLUS $=75$ MINUTOS APÓS BOLUS

Tabela 58 - Valores individuais, média de desvio- padrão da resistência (cmH2O///seg) dos 5 animais do grupo fentanil - São Paulo - 2009

\begin{tabular}{ccccccc}
\hline ANIMAIS & 20 INAL & APBOLUS & 15 BOLUS & 30 BOLUS & 45 BOLUS & 75 BOLUS \\
\hline 1 & 12 & 12 & 12 & 12 & 12 & 12 \\
2 & 10 & 11 & 11 & 11 & 11 & 11 \\
3 & 3 & 2 & 2 & 2 & 2 & 3 \\
4 & 1 & 3 & 3 & 4 & 3 & 4 \\
5 & 9 & 8 & 9 & 8 & 9 & 9 \\
\hline MÉDIA & 12 & 12 & 12 & 12 & 12 & 12 \\
DP & 10 & 11 & 11 & 11 & 11 & 11 \\
\hline
\end{tabular}

20 INAL = 20 MINUTOS APÓS ESTABILIZAÇÃO DA ANESTESIA INALATÓRIA

APBOLUS = IMEDIATAMENTE APÓS O TÉRMINO DO BOLUS

15 BOLUS $=15$ MINUTOS APÓS BOLUS

30 BOLUS $=30$ MINUTOS APÓS BOLUS

45 BOLUS $=45$ MINUTOS APÓS BOLUS

75 BOLUS $=75$ MINUTOS APÓS BOLUS 
Tabela 59 - Valores individuais, média de desvio- padrão da complacência estática das vias aéreas $\left(\mathrm{ml} / \mathrm{cmH}_{2} \mathrm{O}\right)$ dos 5 animais do grupo fentanil - São Paulo 2009

\begin{tabular}{ccccccc}
\hline ANIMAIS & 20 INAL & APBOLUS & 15 BOLUS & 30 BOLUS & 45 BOLUS & 75 BOLUS \\
\hline 1 & 117 & 108 & 108 & 109 & 101 & 101 \\
2 & 128 & 128 & 120 & 116 & 113 & 118 \\
3 & 127 & 140 & 144 & 152 & 153 & 137 \\
4 & 121 & 129 & 129 & 121 & 119 & 115 \\
5 & 177 & 177 & 181 & 175 & 174 & 185 \\
\hline MÉDIA & 134 & 136 & 136 & 135 & 132 & 131 \\
DP & 24,45 & 25,46 & 28,18 & 27,93 & 30,40 & 32,70 \\
\hline
\end{tabular}

20 INAL = 20 MINUTOS APÓS ESTABILIZAÇÃO DA ANESTESIA INALATÓRIA

APBOLUS = IMEDIATAMENTE APÓS O TÉRMINO DO BOLUS

15 BOLUS = 15 MINUTOS APÓS BOLUS

30 BOLUS $=30$ MINUTOS APÓS BOLUS

45 BOLUS $=45$ MINUTOS APÓS BOLUS

75 BOLUS = 75 MINUTOS APÓS BOLUS

Tabela 60 - Valores individuais, média de desvio- padrão da pressão positiva no final da expiração $\left(\mathrm{cmH}_{2} \mathrm{O}\right)$ dos 5 animais do grupo fentanil - São Paulo - 2009

\begin{tabular}{ccccccc}
\hline ANIMAIS & 20 INAL & APBOLUS & 15 BOLUS & 30 BOLUS & 45 BOLUS & 75 BOLUS \\
\hline 1 & 7 & 7 & 7 & 7 & 7 & 7 \\
2 & 7 & 7 & 7 & 7 & 7 & 7 \\
3 & 7 & 7 & 7 & 7 & 7 & 7 \\
4 & 7 & 7 & 7 & 7 & 7 & 7 \\
5 & 7 & 7 & 7 & 7 & 7 & 7 \\
\hline MÉDIA & 7,00 & 7,00 & 7,00 & 7,00 & 7,00 & 7,00 \\
DP & 0,00 & 0,00 & 0,00 & 0,00 & 0,00 & 0,00 \\
\hline
\end{tabular}

20 INAL = 20 MINUTOS APÓS ESTABILIZAÇÃO DA ANESTESIA INALATÓRIA APBOLUS $=$ IMEDIATAMENTE APÓS O TÉRMINO DO BOLUS

15 BOLUS $=15$ MINUTOS APÓS BOLUS

30 BOLUS $=30$ MINUTOS APÓS BOLUS

45 BOLUS $=45$ MINUTOS APÓS BOLUS

75 BOLUS $=75$ MINUTOS APÓS BOLUS 
Tabela 61 - Valores individuais, média de desvio- padrão da pressão de pico inspiratória $\left(\mathrm{cmH}_{2} \mathrm{O}\right)$ dos 5 animais do grupo fentanil - São Paulo - 2009

\begin{tabular}{ccccccc}
\hline ANIMAIS & 20 INAL & APBOLUS & 15 BOLUS & 30 BOLUS & 45 BOLUS & 75 BOLUS \\
\hline 1 & 30 & 30 & 30 & 30 & 30 & 30 \\
2 & 30 & 30 & 30 & 30 & 30 & 30 \\
3 & 30 & 32 & 30 & 32 & 32 & 32 \\
4 & 30 & 29 & 29 & 29 & 30 & 30 \\
5 & 30 & 30 & 29 & 29 & 29 & 29 \\
\hline MÉDIA & 30,00 & 30,20 & 29,60 & 30,00 & 30,20 & 30,20 \\
DP & 0,00 & 1,10 & 0,55 & 1,22 & 1,10 & 1,10 \\
\hline
\end{tabular}

20 INAL = 20 MINUTOS APÓS ESTABILIZAÇÃO DA ANESTESIA INALATÓRIA

APBOLUS = IMEDIATAMENTE APÓS O TÉRMINO DO BOLUS

15 BOLUS $=15$ MINUTOS APÓS BOLUS

30 BOLUS $=30$ MINUTOS APÓS BOLUS

45 BOLUS $=45$ MINUTOS APÓS BOLUS

75 BOLUS = 75 MINUTOS APÓS BOLUS

Tabela 62 - Valores individuais, média de desvio- padrão do CO2 no final da expiração das vias aéreas $(\mathrm{mmHg})$ dos 5 animais do grupo fentanil - São Paulo 2009

\begin{tabular}{ccccccc}
\hline ANIMAIS & 20 INAL & APBOLUS & 15 BOLUS & 30 BOLUS & 45 BOLUS & 75 BOLUS \\
\hline 1 & 39 & 36 & 37 & 36 & 36 & 34 \\
2 & 31 & 32 & 36 & 36 & 33 & 28 \\
3 & 39 & 39 & 39 & 37 & 37 & 40 \\
4 & 34 & 33 & 34 & 34 & 34 & 34 \\
5 & 41 & 40 & 40 & 41 & 39 & 39 \\
\hline MÉDIA & 36,8 & 36 & 37,2 & 36,8 & 35,8 & 35 \\
DP & 4,15 & 3,54 & 2,39 & 2,59 & 2,39 & 4,80 \\
\hline
\end{tabular}

20 INAL = 20 MINUTOS APÓS ESTABILIZAÇÃO DA ANESTESIA INALATÓRIA

APBOLUS = IMEDIATAMENTE APÓS O TÉRMINO DO BOLUS

15 BOLUS $=15$ MINUTOS APÓS BOLUS

30 BOLUS $=30$ MINUTOS APÓS BOLUS

45 BOLUS $=45$ MINUTOS APÓS BOLUS

75 BOLUS $=75$ MINUTOS APÓS BOLUS 
Tabela 63 - Valores individuais, média de desvio- padrão do pH do sangue arterial dos 5 animais do grupo fentanil - São Paulo - 2009

\begin{tabular}{ccccccc}
\hline ANIMAIS & 20 INAL & APBOLUS & 15 BOLUS & 30 BOLUS & 45 BOLUS & 75 BOLUS \\
\hline 1 & 7,31 & 7,35 & 7,32 & 7,33 & 7,34 & 7,37 \\
2 & 7,42 & 7,38 & 7,35 & 7,34 & 7,36 & 7,41 \\
3 & 7,37 & 7,37 & 7,37 & 7,38 & 7,38 & 7,37 \\
4 & 7,38 & 7,40 & 7,40 & 7,41 & 7,39 & 7,40 \\
5 & 7,42 & 7,43 & 7,44 & 7,44 & 7,44 & 7,43 \\
\hline MÉDIA & 7,38 & 7,39 & 7,38 & 7,38 & 7,38 & 7,40 \\
DP & 0,05 & 0,03 & 0,05 & 0,05 & 0,04 & 0,03 \\
\hline
\end{tabular}

20 INAL = 20 MINUTOS APÓS ESTABILIZAÇÃO DA ANESTESIA INALATÓRIA

APBOLUS = IMEDIATAMENTE APÓS O TÉRMINO DO BOLUS

15 BOLUS $=15$ MINUTOS APÓS BOLUS

30 BOLUS $=30$ MINUTOS APÓS BOLUS

45 BOLUS $=45$ MINUTOS APÓS BOLUS

75 BOLUS $=75$ MINUTOS APÓS BOLUS

Tabela 64 - Valores individuais, média de desvio- padrão do bicarbonato no sangue arterial (mmol/l) dos 5 animais do grupo fentanil - São Paulo - 2009

\begin{tabular}{ccccccc}
\hline ANIMAIS & 20 INAL & APBOLUS & 15 BOLUS & 30 BOLUS & 45 BOLUS & 75 BOLUS \\
\hline 1 & 25 & 25 & 26 & 27 & 28 & 28 \\
2 & 25 & 26 & 24 & 26 & 25 & 23 \\
3 & 25 & 24 & 26 & 27 & 27 & 25 \\
4 & 25 & 28 & 28 & 28 & 29 & 30 \\
5 & 28 & 30 & 20 & 28 & 25 & 30 \\
\hline MÉDIA & 25,6 & 26,6 & 24,8 & 27,2 & 26,8 & 27,2 \\
DP & 1,34 & 2,41 & 3,03 & 0,84 & 1,79 & 3,11 \\
\hline
\end{tabular}

20 INAL = 20 MINUTOS APÓS ESTABILIZAÇÃO DA ANESTESIA INALATÓRIA

APBOLUS $=$ IMEDIATAMENTE APÓS O TÉRMINO DO BOLUS

15 BOLUS $=15$ MINUTOS APÓS BOLUS

30 BOLUS $=30$ MINUTOS APÓS BOLUS

45 BOLUS $=45$ MINUTOS APÓS BOLUS

75 BOLUS $=75$ MINUTOS APÓS BOLUS 
Tabela 65 - Valores individuais, média de desvio-padrão do excesso de base no sangue arterial (mmol/l) dos 5 animais do grupo fentanil - São Paulo - 2009

\begin{tabular}{ccccccc}
\hline ANIMAIS & 20 INAL & APBOLUS & 15 BOLUS & 30 BOLUS & 45 BOLUS & 75 BOLUS \\
\hline 1 & -1 & 0 & 0 & 1 & 1 & 2 \\
2 & 1 & 1 & -1 & 0 & 0 & 0 \\
3 & 0 & 0 & 1 & 2 & 2 & 0 \\
4 & 1 & 3 & 3 & 4 & 3 & 4 \\
5 & 3 & 6 & -3 & 4 & 1 & 5 \\
\hline MÉDIA & 0,80 & 2,00 & 0,00 & 2,20 & 1,40 & 2,20 \\
DP & 1,48 & 2,55 & 2,24 & 1,79 & 1,14 & 2,28 \\
\hline
\end{tabular}

20 INAL = 20 MINUTOS APÓS ESTABILIZAÇÃO DA ANESTESIA INALATÓRIA

APBOLUS = IMEDIATAMENTE APÓS O TÉRMINO DO BOLUS

15 BOLUS $=15$ MINUTOS APÓS BOLUS

30 BOLUS $=30$ MINUTOS APÓS BOLUS

45 BOLUS $=45$ MINUTOS APÓS BOLUS

75 BOLUS $=75$ MINUTOS APÓS BOLUS

Tabela 66 - Valores individuais, média de desvio- padrão da temperatura $\left({ }^{\circ} \mathrm{C}\right)$ dos 5 animais do grupo fentanil - São Paulo - 2009

\begin{tabular}{ccccccc}
\hline ANIMAIS & 20 INAL & APBOLUS & 15 BOLUS & 30 BOLUS & 45 BOLUS & 75 BOLUS \\
\hline 1 & 37,8 & 37,3 & 37,3 & 36,7 & 37,2 & 36,1 \\
2 & 37,4 & 37,5 & 36,9 & 37,2 & 36,9 & 36,7 \\
3 & 38,4 & 38,3 & 37,6 & 37,4 & 37,4 & 37,2 \\
4 & 38,3 & 38,1 & 37,8 & 37,5 & 37,2 & 36,4 \\
5 & 36,7 & 36,7 & 36,4 & 36,2 & 35,9 & 36 \\
\hline MÉDIA & 37,7 & 37,6 & 37,2 & 37,0 & 36,9 & 36,5 \\
DP & 0,70 & 0,64 & 0,56 & 0,54 & 0,60 & 0,49 \\
\hline
\end{tabular}

20 INAL = 20 MINUTOS APÓS ESTABILIZAÇÃO DA ANESTESIA INALATÓRIA

APBOLUS = IMEDIATAMENTE APÓS O TÉRMINO DO BOLUS

15 BOLUS $=15$ MINUTOS APÓS BOLUS

30 BOLUS $=30$ MINUTOS APÓS BOLUS

45 BOLUS $=45$ MINUTOS APÓS BOLUS

75 BOLUS $=75$ MINUTOS APÓS BOLUS 


\section{APÊNCICE D - TABELAS RELACIONADAS AO GRUPO ISOFLORANO (GI)}

Tabela 67 - Valores individuais, média e desvio-padrão do peso $(\mathrm{kg})$, débito urinário (ml), e taxa de infusão de fluido, Ringer com Lactato de Sódio (I),dos 5 animais do grupo isoflurano - São Paulo - 2009

\begin{tabular}{cccc}
\hline ANIMAIS & $\begin{array}{c}\text { PESO } \\
(\mathrm{Kg})\end{array}$ & $\begin{array}{c}\text { DU } \\
(\mathrm{ml})\end{array}$ & $\begin{array}{c}\text { FLUIDO } \\
(\mathrm{L})\end{array}$ \\
\hline 1 & 470 & 3000 & 13 \\
2 & 300 & 600 & 14 \\
3 & 540 & 2600 & 11 \\
4 & 500 & 2000 & 8 \\
5 & 580 & 3700 & 13 \\
\hline MÉDIA & 478 & 2380 & 11,8 \\
DP & 107,80 & 1171,32 & 2,65 \\
\hline
\end{tabular}

\section{Parâmetros Hemodinâmicos Diretos}

Tabela 68 - Valores individuais, média de desvio- padrão da freqüência cardíaca (bpm) dos 5 animais do grupo isoflurano - São Paulo - 2009

\begin{tabular}{ccccccc}
\hline ANIMAIS & 20 INAL & APBOLUS & 15 BOLUS & 30 BOLUS & 45 BOLUS & 75 BOLUS \\
\hline 1 & 33 & 35 & 48 & 48 & 50 & 47 \\
2 & 35 & 34 & 36 & 39 & 40 & 36 \\
3 & 29 & 33 & 40 & 44 & 45 & 49 \\
4 & 35 & 35 & 33 & 39 & 45 & 47 \\
5 & 37 & 37 & 40 & 42 & 45 & 45 \\
\hline MÉDIA & 33,8 & 34,8 & 39,4 & 42,4 & 45,0 & 44,8 \\
DP & 3,03 & 1,48 & 5,64 & 3,78 & 3,54 & 5,12 \\
\hline
\end{tabular}

20 INAL = 20 MINUTOS APÓS ESTABILIZAÇÃO DA ANESTESIA INALATÓRIA

APBOLUS = IMEDIATAMENTE APÓS O TÉRMINO DO BOLUS

15 BOLUS $=15$ MINUTOS APÓS BOLUS

30 BOLUS $=30$ MINUTOS APÓS BOLUS

45 BOLUS $=45$ MINUTOS APÓS BOLUS

75 BOLUS $=75$ MINUTOS APÓS BOLUS 
Tabela 69 - Valores individuais, média de desvio- padrão da pressão arterial sistólica $(\mathrm{mmHg})$ dos 5 animais do grupo isoflurano - São Paulo - 2009

\begin{tabular}{ccccccc}
\hline ANIMAIS & 20 INAL & APBOLUS & 15 BOLUS & 30 BOLUS & 45 BOLUS & 75 BOLUS \\
\hline 1 & 71 & 80 & 73 & 83 & 77 & 84 \\
2 & 80 & 87 & 97 & 116 & 106 & 93 \\
3 & 72 & 76 & 93 & 94 & 100 & 120 \\
4 & 77 & 82 & 87 & 87 & 95 & 101 \\
5 & 79 & 65 & 76 & 86 & 76 & 78 \\
\hline MÉDIA & 75,80 & 78,00 & 85,20 & 93,20 & 90,80 & 95,20 \\
DP & 4,09 & 8,28 & 10,45 & 13,37 & 13,63 & 16,39 \\
\hline
\end{tabular}

20 INAL = 20 MINUTOS APÓS ESTABILIZAÇÃO DA ANESTESIA INALATÓRIA

APBOLUS = IMEDIATAMENTE APÓS O TÉRMINO DO BOLUS

15 BOLUS = 15 MINUTOS APÓS BOLUS

30 BOLUS $=30$ MINUTOS APÓS BOLUS

45 BOLUS $=45$ MINUTOS APÓS BOLUS

75 BOLUS = 75 MINUTOS APÓS BOLUS

Tabela $70--$ Valores individuais, média de desvio- padrão da pressão arterial média $(\mathrm{mmHg})$ dos 5 animais do grupo isoflurano - São Paulo - 2009

\begin{tabular}{ccccccc}
\hline ANIMAIS & 20 INAL & APBOLUS & 15 BOLUS & 30 BOLUS & 45 BOLUS & 75 BOLUS \\
\hline 1 & 50 & 56 & 52 & 61 & 57 & 63 \\
2 & 52 & 60 & 65 & 79 & 74 & 73 \\
3 & 47 & 61 & 70 & 65 & 70 & 97 \\
4 & 53 & 58 & 61 & 63 & 74 & 82 \\
5 & 60 & 54 & 58 & 68 & 60 & 64 \\
\hline MÉDIA & 52,40 & 57,80 & 61,20 & 67,20 & 67,00 & 75,80 \\
DP & 4,83 & 2,86 & 6,83 & 7,09 & 8,00 & 14,13 \\
\hline
\end{tabular}

20 INAL = 20 MINUTOS APÓS ESTABILIZAÇÃO DA ANESTESIA INALATÓRIA

APBOLUS = IMEDIATAMENTE APÓS O TÉRMINO DO BOLUS

15 BOLUS = 15 MINUTOS APÓS BOLUS

30 BOLUS $=30$ MINUTOS APÓS BOLUS

45 BOLUS $=45$ MINUTOS APÓS BOLUS

75 BOLUS = 75 MINUTOS APÓS BOLUS 
Tabela 71 - - Valores individuais, média de desvio- padrão da pressão arterial diastólica (mmHg) dos 5 animais do grupo isoflurano - São Paulo - 2009

\begin{tabular}{ccccccc}
\hline ANIMAIS & 20 INAL & APBOLUS & 15 BOLUS & 30 BOLUS & 45 BOLUS & 75 BOLUS \\
\hline 1 & 49 & 44 & 42 & 50 & 47 & 53 \\
2 & 38 & 44 & 47 & 56 & 53 & 57 \\
3 & 35 & 50 & 58 & 51 & 54 & 81 \\
4 & 42 & 46 & 48 & 50 & 61 & 69 \\
5 & 49 & 46 & 48 & 60 & 53 & 56 \\
\hline MÉDIA & 42,60 & 46,00 & 48,60 & 53,40 & 53,60 & 63,20 \\
DP & 6,35 & 2,45 & 5,81 & 4,45 & 4,98 & 11,67 \\
\hline
\end{tabular}

20 INAL = 20 MINUTOS APÓS ESTABILIZAÇÃO DA ANESTESIA INALATÓRIA

APBOLUS = IMEDIATAMENTE APÓS O TÉRMINO DO BOLUS

15 BOLUS = 15 MINUTOS APÓS BOLUS

30 BOLUS $=30$ MINUTOS APÓS BOLUS

45 BOLUS = 45 MINUTOS APÓS BOLUS

75 BOLUS = 75 MINUTOS APÓS BOLUS

\section{Parâmetros de Ventilação, Metabólicos e de Temperatura}

Tabela 72 - Valores individuais, média de desvio- padrão da pressão parcial de $\mathrm{O}_{2}$ no sangue arterial $(\mathrm{mmHg})$ dos 5 animais do grupo isoflurano - São Paulo 2009

\begin{tabular}{ccccccc}
\hline ANIMAIS & 20 INAL & APBOLUS & 15 BOLUS & 30 BOLUS & 45 BOLUS & 75 BOLUS \\
\hline 1 & 378 & 392 & 385 & 395 & 416 & 421 \\
2 & 416 & 391 & 419 & 424 & 407 & 350 \\
3 & 352 & 338 & 339 & 327 & 322 & 313 \\
4 & 310 & 333 & 330 & 333 & 335 & 327 \\
5 & 100 & 127 & 145 & 131 & 142 & 127 \\
\hline MÉDIA & 311 & 316 & 324 & 322 & 324 & 308 \\
DP & 124,21 & 109,42 & 106,12 & 114,41 & 110,23 & 109,18 \\
\hline
\end{tabular}

20 INAL = 20 MINUTOS APÓS ESTABILIZAÇÃO DA ANESTESIA INALATÓRIA

APBOLUS = IMEDIATAMENTE APÓS O TÉRMINO DO BOLUS

15 BOLUS $=15$ MINUTOS APÓS BOLUS

30 BOLUS $=30$ MINUTOS APÓS BOLUS

45 BOLUS $=45$ MINUTOS APÓS BOLUS

75 BOLUS $=75$ MINUTOS APÓS BOLUS 
Tabela 73 - Valores individuais, média de desvio- padrão da saturação arterial de $\mathrm{O}_{2}(\%)$ dos 5 animais do grupo isoflurano - São Paulo - 2009

\begin{tabular}{ccccccc}
\hline ANIMAIS & 20 INAL & APBOLUS & 15 BOLUS & 30 BOLUS & 45 BOLUS & 75 BOLUS \\
\hline 1 & 100 & 100 & 100 & 100 & 100 & 100 \\
2 & 100 & 100 & 100 & 100 & 100 & 100 \\
3 & 100 & 100 & 100 & 100 & 100 & 93 \\
4 & 100 & 100 & 100 & 100 & 100 & 100 \\
5 & 96 & 98 & 98 & 99 & 98 & 98 \\
\hline MÉDIA & 99 & 100 & 100 & 100 & 100 & 98 \\
DP & 1,79 & 0,89 & 0,89 & 0,45 & 0,89 & 3,03 \\
\hline
\end{tabular}

20 INAL = 20 MINUTOS APÓS ESTABILIZAÇÃO DA ANESTESIA INALATÓRIA

APBOLUS $=$ IMEDIATAMENTE APÓS O TÉRMINO DO BOLUS

15 BOLUS $=15$ MINUTOS APÓS BOLUS

30 BOLUS $=30$ MINUTOS APÓS BOLUS

45 BOLUS $=45$ MINUTOS APÓS BOLUS

75 BOLUS = 75 MINUTOS APÓS BOLUS

Tabela 74 - Valores individuais, média de desvio- padrão da pressão parcial do $\mathrm{CO}_{2}$ no sangue arterial $(\mathrm{mmHg})$ dos 5 animais do grupo isoflurano - São Paulo 2009

\begin{tabular}{ccccccc}
\hline ANIMAIS & 20 INAL & APBOLUS & 15 BOLUS & 30 BOLUS & 45 BOLUS & 75 BOLUS \\
\hline 1 & 49 & 48 & 48 & 49 & 49 & 50 \\
2 & 38 & 49 & 47 & 45 & 48 & 47 \\
3 & 39 & 45 & 46 & 43 & 44 & 45 \\
4 & 55 & 50 & 50 & 51 & 53 & 58 \\
5 & 38 & 48 & 48 & 47 & 48 & 50 \\
\hline MÉDIA & 43,8 & 48,0 & 47,8 & 47,0 & 48,4 & 50,0 \\
DP & 7,79 & 1,87 & 1,48 & 3,16 & 3,21 & 4,95 \\
\hline
\end{tabular}

20 INAL = 20 MINUTOS APÓS ESTABILIZAÇÃO DA ANESTESIA INALATÓRIA

APBOLUS = IMEDIATAMENTE APÓS O TÉRMINO DO BOLUS

15 BOLUS = 15 MINUTOS APÓS BOLUS

30 BOLUS $=30$ MINUTOS APÓS BOLUS

45 BOLUS $=45$ MINUTOS APÓS BOLUS

75 BOLUS $=75$ MINUTOS APÓS BOLUS 
Tabela 75 - Valores individuais, média de desvio- padrão da frequência respiratória (mpm) dos 5 animais do grupo isoflurano - São Paulo - 2009

\begin{tabular}{ccccccc}
\hline ANIMAIS & 20 INAL & APBOLUS & 15 BOLUS & 30 BOLUS & 45 BOLUS & 75 BOLUS \\
\hline 1 & 10 & 10 & 10 & 10 & 10 & 10 \\
2 & 10 & 8 & 8 & 8 & 8 & 8 \\
3 & 10 & 10 & 8 & 8 & 8 & 9 \\
4 & 10 & 10 & 10 & 10 & 10 & 10 \\
5 & 10 & 10 & 10 & 10 & 10 & 10 \\
\hline MÉDIA & 10,00 & 9,60 & 9,20 & 9,20 & 9,20 & 9,40 \\
DP & 0,00 & 0,89 & 1,10 & 1,10 & 1,10 & 0,89 \\
\hline
\end{tabular}

20 INAL = 20 MINUTOS APÓS ESTABILIZAÇÃO DA ANESTESIA INALATÓRIA

APBOLUS = IMEDIATAMENTE APÓS O TÉRMINO DO BOLUS

15 BOLUS $=15$ MINUTOS APÓS BOLUS

30 BOLUS $=30$ MINUTOS APÓS BOLUS

45 BOLUS $=45$ MINUTOS APÓS BOLUS

75 BOLUS $=75$ MINUTOS APÓS BOLUS

Tabela 76 - Valores individuais, média de desvio- padrão da concentração do anestésico inalatório no ar expirado (V\%) dos 5 animais do grupo isoflurano - São Paulo - 2009

\begin{tabular}{ccccccc}
\hline ANIMAIS & 20 INAL & APBOLUS & 15 BOLUS & 30 BOLUS & 45 BOLUS & 75 BOLUS \\
\hline 1 & 1,2 & 1,2 & 1,6 & 1,5 & 1,5 & 1,6 \\
2 & 1,4 & 1,5 & 1,3 & 1,8 & 1,5 & 1,7 \\
3 & 1,7 & 1,1 & 1,6 & 1,5 & 1,5 & 1,6 \\
4 & 1,6 & 1,6 & 1,7 & 1,8 & 1,6 & 1,8 \\
5 & 1,5 & 1,5 & 1,9 & 2,1 & 2,1 & 1,8 \\
\hline MÉDIA & 1,5 & 1,4 & 1,6 & 1,7 & 1,6 & 1,7 \\
DP & 0,19 & 0,22 & 0,22 & 0,25 & 0,26 & 0,10 \\
\hline
\end{tabular}

20 INAL = 20 MINUTOS APÓS ESTABILIZAÇÃO DA ANESTESIA INALATÓRIA

APBOLUS = IMEDIATAMENTE APÓS O TÉRMINO DO BOLUS

15 BOLUS $=15$ MINUTOS APÓS BOLUS

30 BOLUS $=30$ MINUTOS APÓS BOLUS

45 BOLUS $=45$ MINUTOS APÓS BOLUS

75 BOLUS $=75$ MINUTOS APÓS BOLUS 
Tabela 77 - Valores individuais, média de desvio- padrão do volume inspiratório (I) dos 5 animais do grupo isoflurano - São Paulo - 2009

\begin{tabular}{ccccccc}
\hline ANIMAIS & 20 INAL & APBOLUS & 15 BOLUS & 30 BOLUS & 45 BOLUS & 75 BOLUS \\
\hline 1 & 6,5 & 6,5 & 6,5 & 6,5 & 6,5 & 6,5 \\
2 & 4 & 4 & 4 & 4 & 4 & 4 \\
3 & 8 & 8 & 9 & 9 & 9 & 10 \\
4 & 7 & 7 & 7 & 7 & 7 & 7 \\
5 & 7 & 8 & 8 & 8 & 8 & 9 \\
\hline MÉDIA & 6,5 & 6,7 & 6,9 & 6,9 & 6,9 & 7,3 \\
DP & 1,50 & 1,64 & 1,88 & 1,88 & 1,88 & 2,33 \\
\hline
\end{tabular}

20 INAL = 20 MINUTOS APÓS ESTABILIZAÇÃO DA ANESTESIA INALATÓRIA

APBOLUS $=$ IMEDIATAMENTE APÓS O TÉRMINO DO BOLUS

15 BOLUS $=15$ MINUTOS APÓS BOLUS

30 BOLUS $=30$ MINUTOS APÓS BOLUS

45 BOLUS $=45$ MINUTOS APÓS BOLUS

75 BOLUS = 75 MINUTOS APÓS BOLUS

Tabela 78 - Valores individuais, média de desvio- padrão do volume expiratório (I) dos 5 animais do grupo isoflurano - São Paulo - 2009

\begin{tabular}{ccccccc}
\hline ANIMAIS & 20 INAL & APBOLUS & 15 BOLUS & 30 BOLUS & 45 BOLUS & 75 BOLUS \\
\hline 1 & 6,39 & 6,30 & 6,68 & 6,52 & 6,57 & 6,50 \\
2 & 4,45 & 4,48 & 4,98 & 5,21 & 4,83 & 4,86 \\
3 & 6,99 & 6,91 & 8,19 & 8,06 & 7,94 & 7,70 \\
4 & 6,85 & 6,84 & 6,81 & 6,77 & 6,63 & 6,78 \\
5 & 6,05 & 6,42 & 6,43 & 6,26 & 5,40 & 6,64 \\
\hline MÉDIA & 6,15 & 6,19 & 6,62 & 6,56 & 6,27 & 6,50 \\
DP & 1,02 & 0,99 & 1,14 & 1,03 & 1,21 & 1,03 \\
\hline
\end{tabular}

20 INAL = 20 MINUTOS APÓS ESTABILIZAÇÃO DA ANESTESIA INALATÓRIA

APBOLUS = IMEDIATAMENTE APÓS O TÉRMINO DO BOLUS

15 BOLUS = 15 MINUTOS APÓS BOLUS

30 BOLUS $=30$ MINUTOS APÓS BOLUS

45 BOLUS $=45$ MINUTOS APÓS BOLUS

75 BOLUS $=75$ MINUTOS APÓS BOLUS 
Tabela 79 - Valores individuais, média de desvio- padrão do platô $\left(\mathrm{cmH}_{2} \mathrm{O}\right)$ dos 5 animais do grupo isoflurano - São Paulo - 2009

\begin{tabular}{ccccccc}
\hline ANIMAIS & 20 INAL & APBOLUS & 15 BOLUS & 30 BOLUS & 45 BOLUS & 75 BOLUS \\
\hline 1 & 20 & 20 & 21 & 21 & 21 & 20 \\
2 & 22 & 23 & 23 & 24 & 24 & 23 \\
3 & 22 & 22 & 23 & 21 & 24 & 24 \\
4 & 20 & 20 & 20 & 20 & 19 & 19 \\
5 & 18 & 19 & 19 & 19 & 18 & 20 \\
\hline MÉDIA & 20 & 21 & 21 & 21 & 21 & 21 \\
DP & 1,67 & 1,64 & 1,79 & 1,87 & 2,77 & 2,17 \\
\hline
\end{tabular}

20 INAL = 20 MINUTOS APÓS ESTABILIZAÇÃO DA ANESTESIA INALATÓRIA

APBOLUS = IMEDIATAMENTE APÓS O TÉRMINO DO BOLUS

15 BOLUS $=15$ MINUTOS APÓS BOLUS

30 BOLUS $=30$ MINUTOS APÓS BOLUS

45 BOLUS $=45$ MINUTOS APÓS BOLUS

75 BOLUS $=75$ MINUTOS APÓS BOLUS

Tabela 80 - Valores individuais, média de desvio- padrão resistência em vias aéreas $\left(\mathrm{cmH}_{2} \mathrm{O} / / / \mathrm{seg}\right)$ dos 5 animais do grupo isoflurano - São Paulo - 2009

\begin{tabular}{ccccccc}
\hline ANIMAIS & 20 INAL & APBOLUS & 15 BOLUS & 30 BOLUS & 45 BOLUS & 75 BOLUS \\
\hline 1 & 9 & 9 & 8 & 8 & 9 & 9 \\
2 & 12 & 12 & 11 & 14 & 12 & 12 \\
3 & 9 & 9 & 9 & 10 & 10 & 10 \\
4 & 9 & 8 & 9 & 9 & 9 & 9 \\
5 & 7 & 7 & 8 & 8 & 7 & 8 \\
\hline MÉDIA & 9,2 & 9 & 9 & 9,8 & 9,4 & 9,6 \\
DP & 1,60 & 1,67 & 1,10 & 2,23 & 1,62 & 1,36 \\
\hline
\end{tabular}

20 INAL = 20 MINUTOS APÓS ESTABILIZAÇÃO DA ANESTESIA INALATÓRIA

APBOLUS = IMEDIATAMENTE APÓS O TÉRMINO DO BOLUS

15 BOLUS $=15$ MINUTOS APÓS BOLUS

30 BOLUS $=30$ MINUTOS APÓS BOLUS

45 BOLUS $=45$ MINUTOS APÓS BOLUS

75 BOLUS $=75$ MINUTOS APÓS BOLUS 
Tabela 81 - Valores individuais, média de desvio- padrão complacência estática das vias aéreas $\left(\mathrm{ml} / \mathrm{cmH}_{2} \mathrm{O}\right)$ dos 5 animais do grupo isoflurano - São Paulo - 2009

\begin{tabular}{ccccccc}
\hline ANIMAIS & 20 INAL & APBOLUS & 15 BOLUS & 30 BOLUS & 45 BOLUS & 75 BOLUS \\
\hline 1 & 237 & 239 & 235 & 231 & 229 & 225 \\
2 & 117 & 116 & 129 & 124 & 124 & 115 \\
3 & 136 & 137 & 155 & 149 & 155 & 144 \\
4 & 146 & 155 & 140 & 148 & 144 & 144 \\
5 & 139 & 151 & 140 & 141 & 122 & 129 \\
\hline MÉDIA & 155 & 160 & 160 & 159 & 155 & 151 \\
DP & 47,08 & 46,93 & 43,04 & 41,69 & 43,72 & 42,88 \\
\hline
\end{tabular}

20 INAL = 20 MINUTOS APÓS ESTABILIZAÇÃO DA ANESTESIA INALATÓRIA

APBOLUS = IMEDIATAMENTE APÓS O TÉRMINO DO BOLUS

15 BOLUS = 15 MINUTOS APÓS BOLUS

30 BOLUS $=30$ MINUTOS APÓS BOLUS

45 BOLUS $=45$ MINUTOS APÓS BOLUS

75 BOLUS = 75 MINUTOS APÓS BOLUS

Tabela 82 - Valores individuais, média de desvio- padrão da pressão positiva no final da expiração $\left(\mathrm{cmH}_{2} \mathrm{O}\right)$ dos 5 animais do grupo isoflurano - São Paulo - 2009

\begin{tabular}{ccccccc}
\hline ANIMAIS & 20 INAL & APBOLUS & 15 BOLUS & 30 BOLUS & 45 BOLUS & 75 BOLUS \\
\hline 1 & 7 & 7 & 7 & 7 & 7 & 7 \\
2 & 7 & 7 & 7 & 7 & 7 & 7 \\
3 & 7 & 7 & 7 & 7 & 7 & 7 \\
4 & 7 & 7 & 7 & 7 & 7 & 7 \\
5 & 7 & 7 & 7 & 7 & 7 & 7 \\
\hline MÉDIA & 7,00 & 7,00 & 7,00 & 7,00 & 7,00 & 7,00 \\
DP & 0,00 & 0,00 & 0,00 & 0,00 & 0,00 & 0,00 \\
\hline
\end{tabular}

20 INAL = 20 MINUTOS APÓS ESTABILIZAÇÃO DA ANESTESIA INALATÓRIA

APBOLUS = IMEDIATAMENTE APÓS O TÉRMINO DO BOLUS

15 BOLUS = 15 MINUTOS APÓS BOLUS

30 BOLUS $=30$ MINUTOS APÓS BOLUS

45 BOLUS $=45$ MINUTOS APÓS BOLUS

75 BOLUS = 75 MINUTOS APÓS BOLUS 
Tabela 83 - Valores individuais, média de desvio- padrão da pressão de pico inspiratório $\left(\mathrm{cmH}_{2} \mathrm{O}\right)$ dos 5 animais do grupo isoflurano - São Paulo - 2009

\begin{tabular}{ccccccc}
\hline ANIMAIS & 20 INAL & APBOLUS & 15 BOLUS & 30 BOLUS & 45 BOLUS & 75 BOLUS \\
\hline 1 & 25 & 25 & 25 & 25 & 25 & 25 \\
2 & 22 & 24 & 25 & 25 & 26 & 25 \\
3 & 25 & 25 & 25 & 25 & 27 & 30 \\
4 & 25 & 25 & 25 & 25 & 25 & 25 \\
5 & 23 & 24 & 26 & 26 & 26 & 26 \\
\hline MÉDIA & 24,00 & 24,60 & 25,20 & 25,20 & 25,80 & 26,20 \\
DP & 1,26 & 0,49 & 0,40 & 0,40 & 0,75 & 1,94 \\
\hline
\end{tabular}

20 INAL = 20 MINUTOS APÓS ESTABILIZAÇÃO DA ANESTESIA INALATÓRIA

APBOLUS $=$ IMEDIATAMENTE APÓS O TÉRMINO DO BOLUS

15 BOLUS $=15$ MINUTOS APÓS BOLUS

30 BOLUS $=30$ MINUTOS APÓS BOLUS

45 BOLUS $=45$ MINUTOS APÓS BOLUS

75 BOLUS = 75 MINUTOS APÓS BOLUS

Tabela 84 - Valores individuais, média de desvio- padrão do CO2 no final da expiração das vias aéreas $(\mathrm{mmHg})$ dos 5 animais do grupo isoflurano - São Paulo $-2009$

\begin{tabular}{ccccccc}
\hline ANIMAIS & 20 INAL & APBOLUS & 15 BOLUS & 30 BOLUS & 45 BOLUS & 75 BOLUS \\
\hline 1 & 37 & 38 & 36 & 38 & 38 & 38 \\
2 & 33 & 40 & 38 & 37 & 38 & 37 \\
3 & 35 & 37 & 36 & 37 & 38 & 39 \\
4 & 43 & 40 & 40 & 41 & 42 & 43 \\
5 & 44 & 43 & 45 & 45 & 44 & 49 \\
\hline MÉDIA & 38 & 40 & 39 & 40 & 40 & 41 \\
DP & 4,88 & 2,30 & 3,74 & 3,44 & 2,83 & 4,92 \\
\hline
\end{tabular}

20 INAL = 20 MINUTOS APÓS ESTABILIZAÇÃO DA ANESTESIA INALATÓRIA APBOLUS = IMEDIATAMENTE APÓS O TÉRMINO DO BOLUS

15 BOLUS = 15 MINUTOS APÓS BOLUS

30 BOLUS $=30$ MINUTOS APÓS BOLUS

45 BOLUS $=45$ MINUTOS APÓS BOLUS

75 BOLUS $=75$ MINUTOS APÓS BOLUS 
TABELA 85 - Valores individuais, média de desvio- padrão do pH do sangue arterial dos 5 animais do grupo isoflurano - São Paulo - 2009

\begin{tabular}{ccccccc}
\hline ANIMAIS & 20 INAL & APBOLUS & 15 BOLUS & 30 BOLUS & 45 BOLUS & 75 BOLUS \\
\hline 1 & 7,36 & 7,37 & 7,36 & 7,37 & 7,37 & 7,37 \\
2 & 7,33 & 7,26 & 7,27 & 7,27 & 7,27 & 7,28 \\
3 & 7,34 & 7,25 & 7,26 & 7,26 & 7,26 & 7,26 \\
4 & 7,31 & 7,33 & 7,33 & 7,33 & 7,3 & 7,31 \\
5 & 7,40 & 7,31 & 7,31 & 7,31 & 7,30 & 7,30 \\
\hline MÉDIA & 7,35 & 7,30 & 7,31 & 7,31 & 7,30 & 7,30 \\
DP & 0,03 & 0,05 & 0,04 & 0,04 & 0,04 & 0,04 \\
\hline
\end{tabular}

20 INAL = 20 MINUTOS APÓS ESTABILIZAÇÃO DA ANESTESIA INALATÓRIA

APBOLUS = IMEDIATAMENTE APÓS O TÉRMINO DO BOLUS

15 BOLUS = 15 MINUTOS APÓS BOLUS

30 BOLUS $=30$ MINUTOS APÓS BOLUS

45 BOLUS $=45$ MINUTOS APÓS BOLUS

75 BOLUS = 75 MINUTOS APÓS BOLUS

Tabela 86 - Valores individuais, média de desvio- padrão do bicarbonato no sangue arterial $(\mathrm{mmol})$ dos 5 animais do grupo isoflurano - São Paulo - 2009

\begin{tabular}{ccccccc}
\hline ANIMAIS & 20 INAL & APBOLUS & 15 BOLUS & 30 BOLUS & 45 BOLUS & 75 BOLUS \\
\hline 1 & 26 & 27 & 27 & 27 & 28 & 28 \\
2 & 25 & 25 & 25 & 25 & 25 & 26 \\
3 & 27 & 26 & 26 & 27 & 27 & 28 \\
4 & 27 & 26 & 25 & 26 & 25 & 28 \\
5 & 29 & 27 & 29 & 29 & 29 & 30 \\
\hline MÉDIA & 26,8 & 26,2 & 26,4 & 26,8 & 26,8 & 28,0 \\
DP & 1,48 & 0,84 & 1,67 & 1,48 & 1,79 & 1,41 \\
\hline
\end{tabular}

20 INAL = 20 MINUTOS APÓS ESTABILIZAÇÃO DA ANESTESIA INALATÓRIA APBOLUS $=$ IMEDIATAMENTE APÓS O TÉRMINO DO BOLUS

15 BOLUS $=15$ MINUTOS APÓS BOLUS 30 BOLUS $=30$ MINUTOS APÓS BOLUS 45 BOLUS $=45$ MINUTOS APÓS BOLUS 75 BOLUS $=75$ MINUTOS APÓS BOLUS 
Tabela 87 - Valores individuais, média de desvio- padrão do excesso de base no sangue arterial ( $\mathrm{mmol} / \mathrm{l})$ dos 5 animais do grupo isoflurano - São Paulo - 2009

\begin{tabular}{ccccccc}
\hline ANIMAIS & 20 INAL & APBOLUS & 15 BOLUS & 30 BOLUS & 45 BOLUS & 75 BOLUS \\
\hline 1 & 1 & 1 & 1 & 1 & 2 & 2 \\
2 & -1 & -1 & -1 & -1 & -1 & -1 \\
3 & 1 & 0 & 1 & 0 & 0 & 0 \\
4 & 0 & 0 & 0 & 0 & -1 & -1 \\
5 & -1 & 1 & 0 & 1 & 1 & 1 \\
\hline MÉDIA & 0 & 0,2 & 0,2 & 0,2 & 0,2 & 0,2 \\
DP & 1,00 & 0,84 & 0,84 & 0,84 & 1,30 & 1,30 \\
\hline
\end{tabular}

20 INAL = 20 MINUTOS APÓS ESTABILIZAÇÃO DA ANESTESIA INALATÓRIA

APBOLUS = IMEDIATAMENTE APÓS O TÉRMINO DO BOLUS

15 BOLUS = 15 MINUTOS APÓS BOLUS

30 BOLUS $=30$ MINUTOS APÓS BOLUS

45 BOLUS $=45$ MINUTOS APÓS BOLUS

75 BOLUS = 75 MINUTOS APÓS BOLUS

Tabela 88 - Valores individuais, média de desvio- padrão da temperatura $\left({ }^{\circ} \mathrm{C}\right)$ dos 5 animais do grupo isoflurano - São Paulo - 2009

\begin{tabular}{ccccccc}
\hline ANIMAIS & 20 INAL & APBOLUS & 15 BOLUS & 30 BOLUS & 45 BOLUS & 75 BOLUS \\
\hline 1 & 38,3 & 37,9 & 37,4 & 36,9 & 36,5 & 36 \\
2 & 38,3 & 38,3 & 37,9 & 37,7 & 37,4 & 37,1 \\
3 & 38,0 & 38,3 & 37,8 & 37,5 & 37,5 & 37,3 \\
4 & 38,0 & 37,8 & 37,6 & 37,5 & 37,5 & 36,8 \\
5 & 38,1 & 37,7 & 37,6 & 37,4 & 36,8 & 36,6 \\
\hline MÉDIA & 38,14 & 38,00 & 37,66 & 37,40 & 37,14 & 36,76 \\
DP & 0,15 & 0,28 & 0,19 & 0,30 & 0,46 & 0,50 \\
\hline
\end{tabular}

20 INAL = 20 MINUTOS APÓS ESTABILIZAÇÃO DA ANESTESIA INALATÓRIA

APBOLUS = IMEDIATAMENTE APÓS O TÉRMINO DO BOLUS

15 BOLUS $=15$ MINUTOS APÓS BOLUS

30 BOLUS $=30$ MINUTOS APÓS BOLUS

45 BOLUS $=45$ MINUTOS APÓS BOLUS

75 BOLUS = 75 MINUTOS APÓS BOLUS 